九州大学学術情報リポジトリ

Kyushu University Institutional Repository

\title{
Museum archives of the 19 years long time- series sediment trap samples collected at central subarctic Pacific Station SA and Bering Sea Station AB during 1990-2010
}

Takahashi, Kozo

Department of Earth and Planetary Sciences, Graduate School of Sciences, Kyushu University

Asahi, Hirofumi

Atmosphere and Ocean Research Institute, University of Tokyo

Okazaki, Yusuke

Department of Earth and Planetary Sciences, Graduate School of Sciences, Kyushu University I Research Institute for Global Change, JAMSTEC

Onodera, Jonaotaro

Research Institute for Global Change, JAMSTEC

他

https://doi.org/10.5109/21002

出版情報：九州大学大学院理学研究院紀要：Series D, Earth and planetary sciences. 32 (4), pp. 139, 2012-03-01. Faculty of Science, Kyushu University

バージョン :

権利関係 : 
Mem. Fac. Sci., Kyushu Univ., Ser. D, Earth \& Planet. Sci., Vol. XXXII, No. 4, pp. 1-38, March 1, 2012

\title{
Museum archives of the 19 years long time-series sediment trap samples collected at central subarctic Pacific Station SA and Bering Sea Station AB during 1990-2010
}

\author{
Kozo Takahashi*, Hirofumi Asahi**, Yusuke Okazaki* \&***, Jonaotaro Onodera**, Hideto Tsutsui*, \\ Takahito Ikenoue*, Yoshiyuki Kanematsu*, Seiji Tanaka ${ }^{* * * *}$ and Shinya Iwasaki*
}

\begin{abstract}
PARLUX type sediment traps were moored at $600 \mathrm{~m}$ above the sea-floor at Station SA (water depth: 5,406 m) in the central subarctic Pacific and at Station AB (water depth: 3,788 m) in the Aleutian Basin of the Bering Sea. The time-series flux samples were obtained during 1990-2010 for nineteen years. This allowed us to characterize primary fluxes of biogenic particles primarily produced near the surface layers of the deep water columns as well as to decipher the environmental variations associated with climate changes. Based on the samples various studies were conducted thus far, including the followings types: quantitative plankton taxon-numerical studies on diatoms, silicoflagellates, radiolarians, coccolithophores and planktic foraminfers; and geochemical aspects of chemical compounds such as biogenic opal, calcium carbonate and hexosamine as well as elements such as rare earth elements. Such studies published thus far are cited in this paper.

The sediment trap samples are archived at the Kyushu University Museum for permanent preservation. The grand total of the currently archived samples, including membrane filters and microslides for the two stations, is 3,552. The details of the samples are described herein and spreadsheet tables of the archives will also be electronically published by the Museum. This makes it possible for future scientists and students, who need to examine the relevant sediment trap samples, to access them properly.
\end{abstract}

Keywords: Museum archives, sediment trap samples, the central subarctic Pacific, the Bering Sea, Station SA, Station $\mathrm{AB}$, sinking paticles, flux, time-series, seasonal, inter-annual

\section{Introduction}

Collection of time-series sediment trap samples allows us to comprehend detailed behaviors of biogenic particle production which is subsequently sinking into the deep sea. The production of biogenic particles is primarily concentrated in the upper most layers, with some exception in the deeper layers depending on taxa, of the oceans. Many, if not all, of the shell bearing plankton groups such as diatoms and radiolarians are produced attributing to certain environmental conditions and they can be transported to the sea-floor by means of aggregate sinking (e.g., Takahashi, 1986, 1987a, b, c, 1995; Takahashi et al., 1989, 1990; Honjo et al., 2008) and subsequently preserved as microfossils in sediments (e.g., Takahashi, 1994). Therefore, they can be well utilized for reconstruction of the past environmental conditions. With the same token the sinking particles intercepted by sediment traps can be utilized in reconstructing the relatively recent past environmental conditions prevailed at the time of their production.

In global scale Honjo et al. (2008) showed that the region inclusive of Stations SA and AB represents one of

Manuscript received on 20 January 2012; accepted on 6 February 2012

* Department of Earth and Planetary Sciences, Graduate School of Sciences, Kyushu University, Hakozaki 6-10-1, Fukuoka 812-8581, Japan; e-mail: kozo@geo.kyushu-u.ac.jp

** Atmosphere and Ocean Research Institute, University of Tokyo, 5-1-5 Kashiwanoha, Kashiwa, Chiba 277-8564, Japan

*** Research Institute for Global Change, JAMSTEC, 2-15 Natsushima-cho, Yokosuka 237-0061, Japan

****Division of Marine Bioreseource and Environmental Science, Graduate School of Fisheries Sciences, Hokkaido University, 3-1-1, Minato-cho, Hakodate, Hokkaido 041-8611, Japan 
Kozo Takahashi, Hirofumi Asahi, Yusuke Okazaki, Jonaotaro Onodera, Hideto Tsutsui,

Takahito Ikenoue, Yoshiyuki Kanematsu, Seiji Tanaka and Shinya Iwasaki

the highest biogenic opal/ $\mathrm{CaCO}_{3}$ ratios in the world and hence efficient performance of biological pump in the region is evident. Such an efficiency of the biological pump could change with varying environmental conditions such as increase in $\mathrm{PCO}_{2}$, sea surface temperature, and the constituents of $\mathrm{CaCO}_{3}$ bearing plankton taxa. Thus, the time-series sediment trap samples from this particular region represents very important for the years to come, especially when one wishes to compare with the future environmental conditions. It is the authors wish that the sediment trap samples are permanently archived at the Kyushu University Museum. In order to properly archive the samples it is necessary to accompany with well organized documents for the samples. This paper serves as such a required publication.

\subsection{Oceanographic setting}

In the central subarctic Pacific the Alaskan Stream, an extension of the Alaskan Current, flows westward along the Aleutian Islands and enters the Bering Sea partially through the Amchitka Strait and to significant extent through the Near Strait west of Attu Island in the eastern Aleutian Islands (Fig. 1). A part of the Subarctic Current also joins the Alaskan Stream (Ohtani, 1965). Much of the Pacific water entering the Bering Sea is matched by outflow through the Aleutian Islands. The most significant outflow is through the Kamchatka Strait, which has a maximum depth of 4,420 m (Stabeno et al., 1999). Station SA is located approximately in between the pathways of the Subarctic Current and the Alaskan Stream and thus ideal location in monitoring the changes in the extent of both currents (Fig. 1).

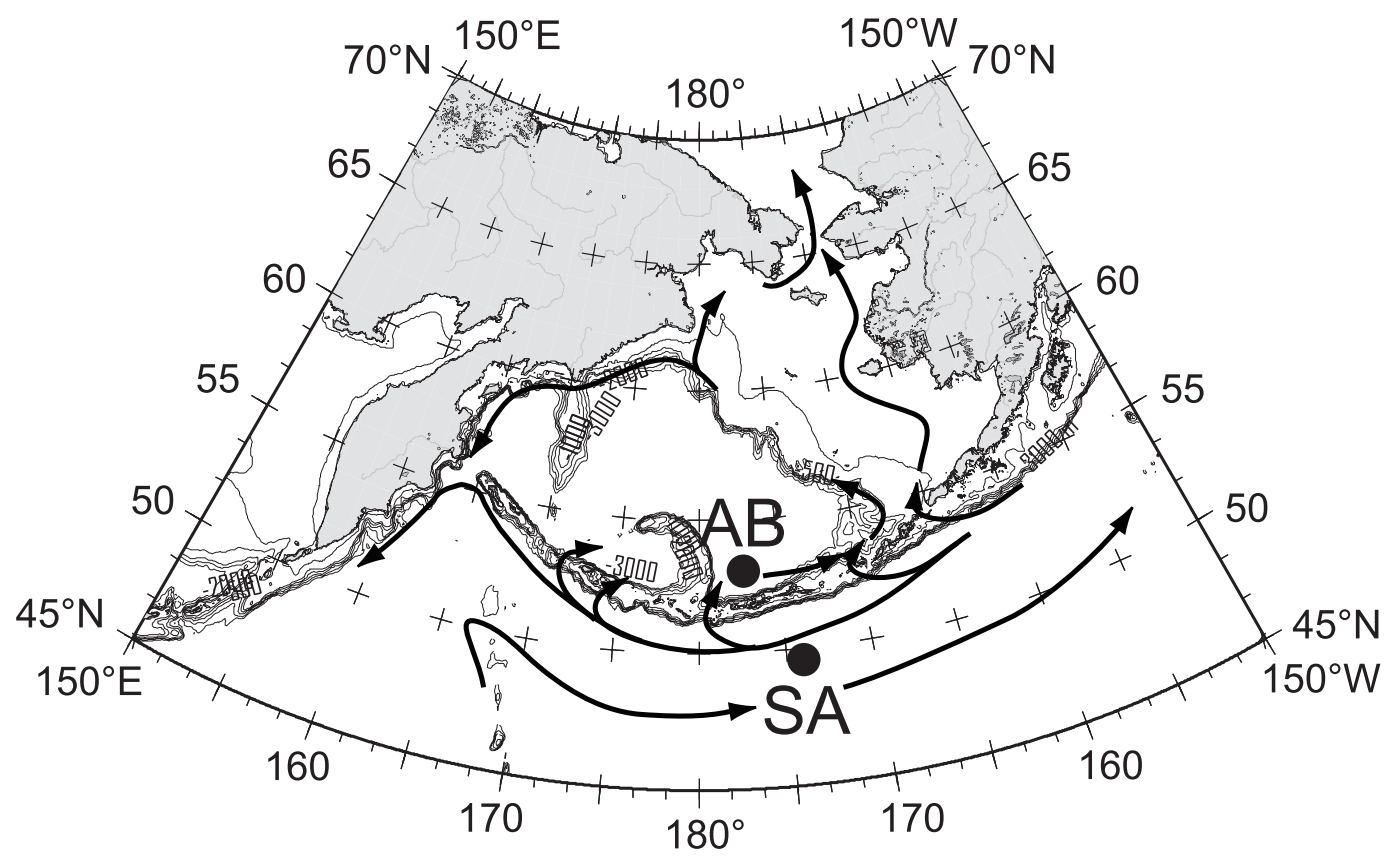

Fig. 1. Map showing the locations of Stations SA and AB. Also included are the bathymetric contour lines as well as major surface currents. The map is drawn based on Collaborative Research Center (SFB) 574 : https://sfb574.ifm-geomar.de/gmt-maps

The Bering Sea is a marginal sea which is semi-enclosed by landmasses of Russian Siberia to the west, Alaska to the east, and the Aleutian Islands to the south. Approximately half of the Bering Sea is a shallow (0-200 m), neritic environment, with the majority of the continental shelf spanning the eastern side of the basin off Alaska from Bristol Bay to the Bering Strait. The Bering Strait is $\sim 50 \mathrm{~m}$ deep today (Takahashi, 2005) and the unidirectional net water flowing towards north through the Strait makes up a part of today's water mass properties of the Canadian Archipelago of the Arctic Ocean as well as that of the Labrador Sea in the Atlantic unique (Reid et al., 2007). Another half of the Bering Sea area is represented by hemipelagic waters in the Bering Basin, which can be split into three smaller basins, the Aleutian, Bowers, and Kamchatka (Komandorsky) Basins. Station AB is located in the southern part of the Aleutian Basin whose water depth is $\sim 3,800 \mathrm{~m}$ (Fig. 1).

\subsection{Sediment trap mooring experiment}

Our initial sediment trap mooring experiment for the fist year began in August 1989 and completed in August 1990 at Station SA $\left(49^{\circ} \mathrm{N}, 174^{\circ} \mathrm{W}\right)$ in the central subarctic Pacific. However, the implosion of one of the glass floats attached to the mooring caused the bottom end of the sediment trap cone plugged by the glass fragments, 
leading to incomplete collection of only trace amount of samples. In spite of such an accident occurred at Station SA during 1989-1990 the subsequent trap deployments/recoveries went fairly well during 1990-2010 for twenty years (nineteen years of actual sample acquisition; for details see Table 2), with several exceptions of missing a small number of samples (Tables 1-3).

\subsection{The value of the trap samples}

The value of the trap samples is immense. This is basically because that our samples came from rather critical time period when, for example, global warming is taking place today (IPCC AR4 SYR, 2007). The nineteen year long period for the sample collection is in the past and hence you are no longer be able to go back in time to take the same set of samples again. Therefore, such a sample set provides unique archives of the environmental proxies such as productivity (e.g., Takahashi et al., 2000, 2002; Onodera and Takahashi, 2009, 2011), temperature (e.g., Asahi and Takahashi, 2007, 2008; Tsutsui et al., submitted) and perhaps many other oceanographic conditions. We are providing the sample archives so that people of the future, for example, even a hundred years later, can properly access to the samples for their use, for instance, to compare with what they will collect at the same stations a hundred years later.

Our plan is to archive relevant time-series sediment samples (hereafter trap samples) permanently at the Kyushu University Museum. In order for the museum curators to properly be able to archive the samples at the Museum it is necessary for us to publish the detailed data base such as the present one organized here.

\subsection{Studies conducted on the trap samples}

A fair number of studies utilizing the trap samples from Stations SA and AB have been conducted thus far: Takahashi et al., 1996, 2000, 2002, 2007; Hashimoto et al., 1998; Kurihara and Takahashi, 2002; Asahi and Takahashi, 2007, 2008; Onodera and Takahashi, 2009, 2011; Onodera et al., 2007, 2009; Akagi et al., 2011; Ikenoue et al., 2012; Tsutsui et al., submitted. Studies concerning total mass, organic carbon (or organic matter), biogenic opal, and calcium carbonate $\left(\mathrm{CaCO}_{3}\right)$ have been backbones of further studies dealing with detailed proxies such as plankton taxa and assemblages; the backbone studies are Takahashi et al. (1997, 2000, 2002, 2007).

Constituents of organic and inorganic elements and compounds have also been of interest to many of us. The works on amino acids and hexosamine were represented by Hashimoto et al. $(1997,1998)$ and Maita et al. $(1996$, 1999). The results on rare earth elements were published by Akagi et al. (2011).

Because that diatoms as primary producers representing by far the largest contribution among many shell bearing sinking plankton assemblages we have been investigating the details. This includes taxon constituents, seasonality, inter-annual flux variability and many other aspects concerning environmental changes occurring in the region. The results for the initial eight years of diatom fluxes have been published by Onodera and Takahashi (2009) and Onodera et al. (2009). Other works on diatoms have also been published: Takahashi et al., 1996; Takahashi, 1999; Onodera et al., 2007. In order to decipher sexual production scheme of Neodenticula seminae, the dominant diatom taxon in the region, biometrics of this taxon have been measured and discussed for eight years by Kurihara and Takahashi (2002).

Silicoflagellates as primary producers constitute a rather minor group in terms of fluxes of skeletons, especially compared with that of diatoms, but they carry important environmental proxy signals. Onodera and Takahashi (2011) conducted the initial four years of silicoflagellate fluxes and obtained relevant results concerning environmental changes occurred in the region.

Radiolarians as microzooplankton constitute by far the most complex siliceous shell bearing sinking flux assemblage due to highly diversified taxa as well as a wide range in their living depth habitats (Takahashi, 1991). Ikenoue et al. (2012) carried out a fifteen year long time-series fluxes of radiolarians and discussed with the changes of climate indices. Earlier on preliminary results on radiolarians were achieved by Itaki and Takahashi (1995) and Itaki et al. (1997). Takahashi (1995) discussed the fate of biogenic opal particle fluxes together with citing earlier results of Cycladophora davisiana, an intermediate depth dwelling radiolarian.

Furthermore, coccolithophores represent the single most important primary producers among $\mathrm{CaCO}_{3}$ shell bearing sinking plankton groups. The work by Tsutsui et al. (submitted) represents nineteen year long fluxes of major taxa including Emiliania huxeleyi and Coccolithus pelagicus. It is of interest to note that a sign of global warming has been detected with the increase in E. huxeleyi first time in sediment trap records (Tsutsui et al., submitted).

Planktic foraminifers, the last taxonomic group described here but not the least, are quite useful environmental tracers of the region and their fluxes appear to fluctuate depending on the temperature variations based on the nine year long trap results (Asahi and Takahashi, 2007). Utilities of multivariate analyses on planktonic foraminifers are discussed by Asahi and Takahashi $(2003,2008)$ and Asahi et al. (2007). 
Kozo Takahashi, Hirofumi Asahi, Yusuke Okazaki, Jonaotaro Onodera, Hideto Tsutsui,

Takahito Ikenoue, Yoshiyuki Kanematsu, Seiji Tanaka and Shinya Iwasaki

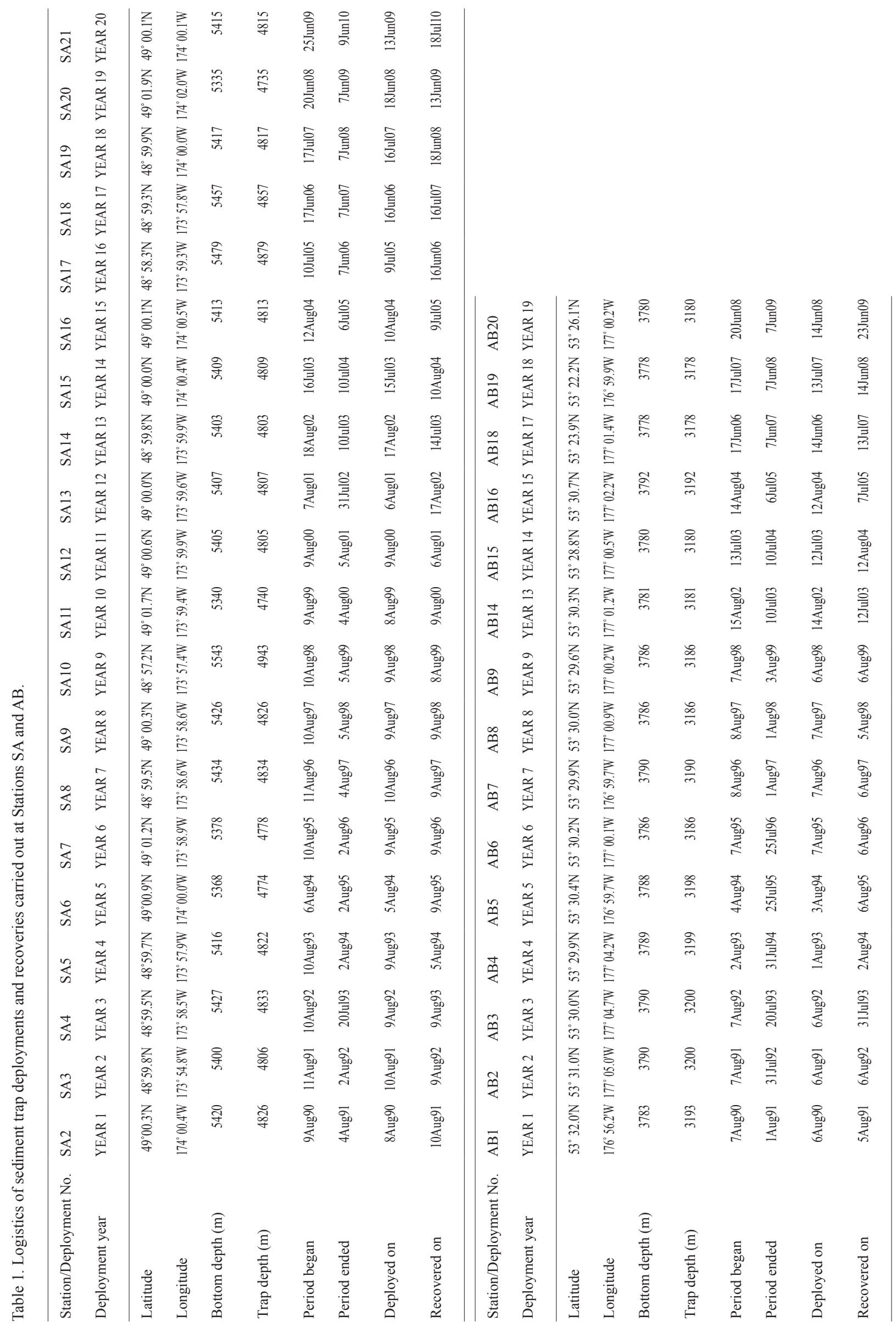




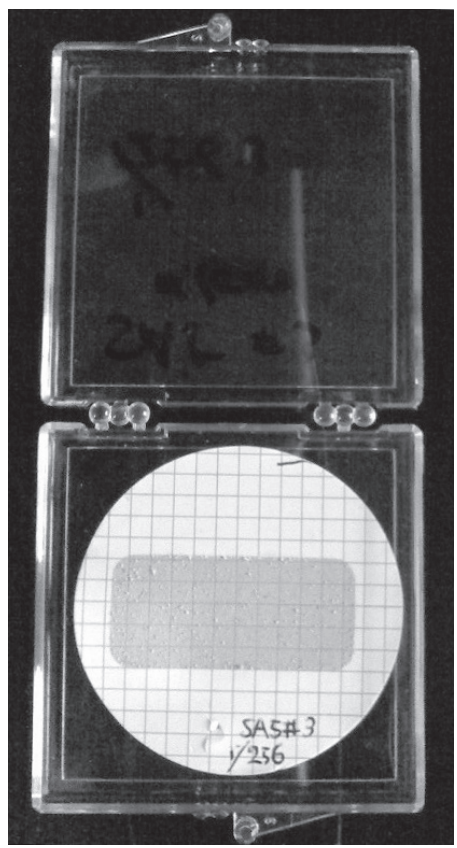

$1 \mathrm{~cm}$

Fig. 2. An example of a dry sample prepared on Gelman ${ }^{\circledR}$ membrane filters together with a hinged plastic box for storage of the sample.

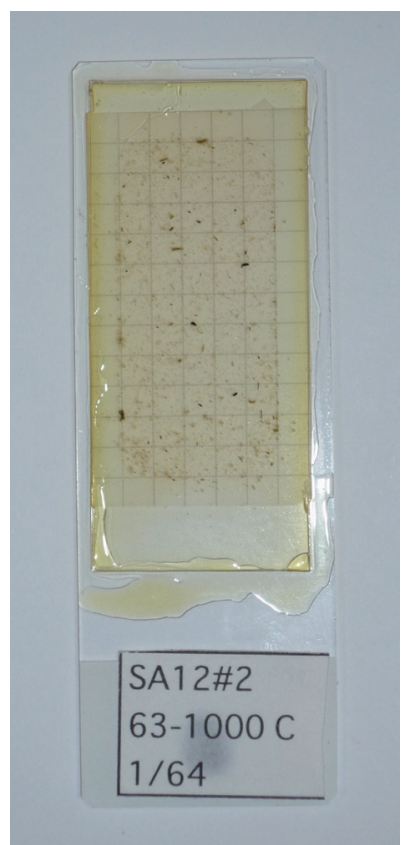

Fig. 3. An example of a microslide.
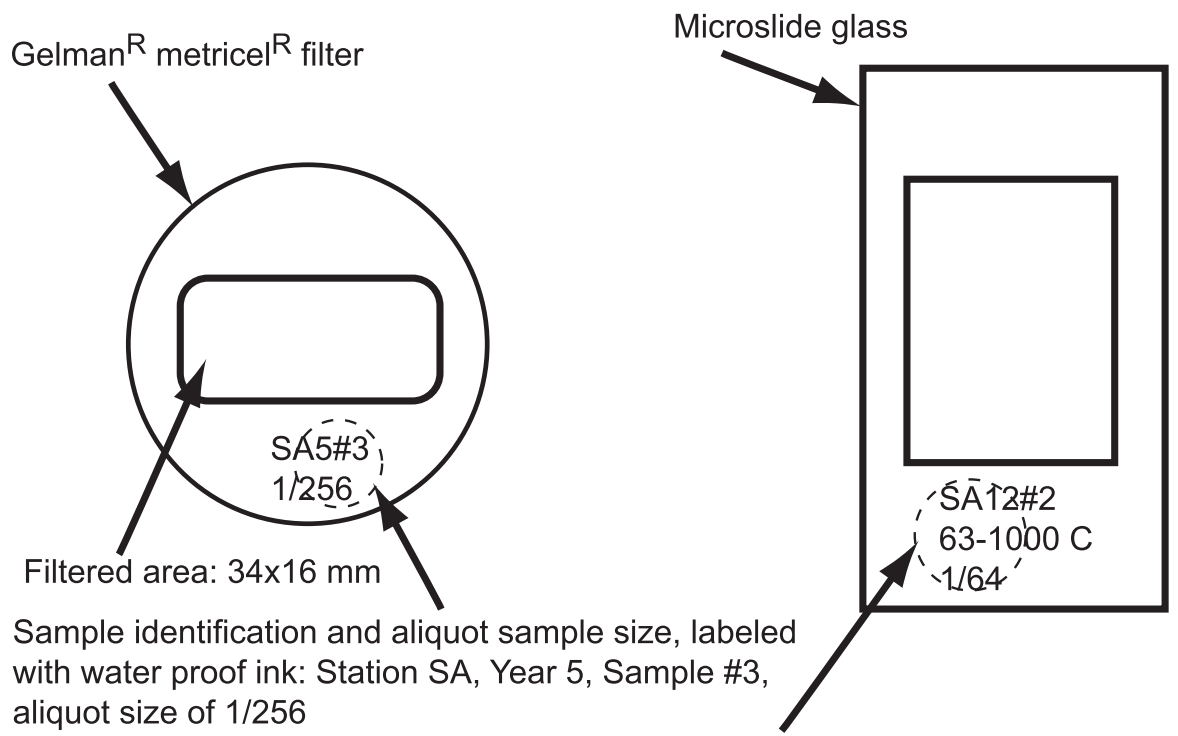

Sample identification and aliquot sample size, labeled with water proof ink: Station SA, Year 5, Sample \#3, aliquot size of $1 / 256$

Sample identification and aliquot size:

Station SA, Year 12, Sample \#2, aliquot size of 1/64

Fig. 4. Illustration showing examples of a label directly written on a Gelman ${ }^{\circledR}$ membrane filter and that placed on a microslide. 


\section{Methods}

\subsection{Sources of samples}

A Honjo type (PARFLUX Mark 7G-13: aperture area: $0.50 \mathrm{~m}^{2} ; 13$ samplers) time-series sediment trap was employed for this study; its full description has been published (Honjo and Doherty, 1988). This trap model had been employed through till the following specified dates at each of the locations: Station SA: for 15 years till June 2005; Station AB: for 12 years till June 2002. Later on the above model had been replaced by PARFLUX Mark 7G-21 which had 21 samplers (see details in Table 1). Thus, with the new model the dates when the new sampling strategy began were the followings: Station SA: for 5 years since July 2005; Station AB: since August 2002 (Table 1).

Deep water samples from 2,000 $\mathrm{m}$ were obtained at each of the stations to prepare preservative solutions to fill the sample bottles. We employed two different preservative solutions depending on the year of the deployments: 5\% glutaraldehyde for initial four years (1990-1994); and 5\% formaldehyde for the last sixteen years (1994-2010). Such solutions were mixed with sodium borate as a buffer (pH 7.6 to 7.8). Our sampling strategy was to target high-resolution sampling such as for 20 days during highly productive spring to fall seasons and a relatively low resolution such as 56 days for the quiescent winter season. The details of exact temporal intervals of the samples are listed (Tables 2-3).

At each of the stations a mooring system with a trap was deployed from T/V Oshoro-Maru of the Hokkaido University and anchored to the sea-floor. Each of the mooring systems was designed to set the trap at approximately $600 \mathrm{~m}$ above the sea-floor. Such a depth of the moored sediment traps was determined in order to avoid possible inclusion of particles supplied by the nepheloid layer up to ca. $400 \mathrm{~m}$ above the seafloor (Ewing and Connary, 1970). The pelagic Station SA $\left(49^{\circ} \mathrm{N}, 174^{\circ} \mathrm{W}\right)$ is located just south of the Aleutian Islands and Aleutian Trench in the central subarctic Pacific and its water depth is 5,406 m (Fig. 1; Table 1). A sediment trap was deployed at 4,812 m. Data for all 1990-2010 are presented here to directly compare with those at Station AB. During the 1989-1990 first year deployment (designated as YEAR minus 1), the bottom end of the trap funnel was plugged with glass fragments due to an accidental implosion of a glass sphere placed approximately $22 \mathrm{~m}$ directly above the trap. Thus, only limited flux samples were obtained during this period and the information is not included in this paper. The information from 1990 to 2010 (designated as YEARs 1-20 in this paper) of the actual trap deployments, is reported here.

The marginal sea Station $\mathrm{AB}\left(53.53^{\circ} \mathrm{N}, 1773^{\circ} \mathrm{W}\right)$ is located in the Aleutian Basin of the Bering Sea at a water depth of 3,788 m (Fig. 1; Table 1). This is a representative station for the Aleutian Basin. The sediment traps of the same types as those used at Station SA were deployed here at 3,198 m from August 1990 through June 2010 (YEARs 1-20). The two trap stations in the subarctic region are $530 \mathrm{~km}$ apart each other and located in distinctively different environments: "pelagic" vs "marginal sea".

The disturbance of the sedimentation of particles by currents was considered. In fact, we deployed a current meter (made by Aanderaa) placed 3 m below the sediment trap at Station SA during August 1989-1994. The results clearly suggested that the currents were weak (maximum of $3 \mathrm{~cm} \mathrm{~s}^{-1}$ : H. Miyake, unpublished data). Based on the current meter measurements, we conclude that the advective currents were negligibly small and they did not significantly affect the fluxes intercepted by the trap.

\subsection{Sample identification numbers}

The identification numbers for the trap samples are given; each table is organized for a single station for simplicity (Tables 2-3). More or less continuous time-series samples are available at Station SA, except for the bulk of samples during 2009-2010. This makes a collection of trap samples at Station SA for full 19 years since August 1990 (Table 2). A more irregular availability of samples is a reality at Station AB. No sample is available for YEARs 10-12, 16, and 20 at Station AB due to hiatuses in trap deployment or unsuccessful trap mooring recovery. Starting from the trap YEAR 13 in 2002 at Station AB the sample identification was designated as AB14, by skipping AB13 (Table 3). This was to synchronize the deployment year identification with that of Station SA, especially with the hiatuses of three years (YEARs 10-12) prior to YEAR 13. Note that SA14 and AB14 coincide for the deployments during 2002-2003. For more details see Tables 2-3.

\subsection{Sample splitting and sieving}

The recovered trap samples were kept refrigerated on board until the ship arrived at Hakodate Port, Japan. First, a whole sample was sieved through a $1 \mathrm{~mm}$ stainless steel mesh and then split with a rotary liquid sample splitter into 1/4 aliquots on board while the ship was in port. An exception is that during the initial three years for 1990-1993 the sieving process through a $63 \mu \mathrm{m}$ stainless steel mesh was applied "prior to splitting". Such a sieving of a fair amount of sample through the fine mesh was, however, determined to be extremely difficult and hence an "initial $63 \mu \mathrm{m}$ sieving" procedure was discontinued at the fourth year recovery. The alternative 
procedure starting from the fourth year and continued on to the successive years was to "sieve after splitting" into $1 / 4$ aliquot size. This is how the two fractions were prepared after the initial three years: coarse fraction: $1 \mathrm{~mm}-63$ $\mu \mathrm{m}$; and fine fraction: $<63 \mu \mathrm{m}$. The buffered $5 \%$ glutardehyde (formalin beginning from YEAR 5) solution was used to split the whole sample. This was not to change the concentration of the buffered preservatives. Two of the 1/4 aliquots were brought to each of the two separate shore laboratories (one lab. initially at Woods Hole Oceanographic Institution, Woods Hole, MA USA, and later moved to Hokkaido Tokai University, Sapporo, and subsequently to Kyushu University, Fukuoka; the other at Hokkaido University, Hakodate, Japan) and the aliquots were kept refrigerated until they were subjected to further splitting and subsequent analyses.

Apparent swimmers were removed by hand-picking under a dissecting microscope. Generally, there were several crustaceans in the $>1 \mathrm{~mm}$ fractions (mega fraction); they were assumed to be swimmers since they were well preserved and sometimes with bright red color inidicating that they were alive at the time of entry to the trap sample bottle. In the $63 \mu \mathrm{m}-1 \mathrm{~mm}$ fraction (coarse fraction), usually no visible crustaceans were spotted during sieving and thus most particles in the samples were left alone. The measured fluxes in the mega fraction turned out to be insignificant in weight compared with those of the coarse and fine $(<63 \mu \mathrm{m})$ fractions. Therefore, the sum of the coarse and fine fractions is reported in Takahashi et al. $(1997,2000,2002,2007)$. The mega fraction contains mainly phaeodarian radiolarians (Takahashi et al., 1983).

\subsection{Examples of microslide preparation and counting procedures for taxon-quantitative assessment of coccolithophores and radiolarians}

It is a good idea to illustrate two examples of microslide preparations and counting procedures for taxonquantitative assessment of the particle fluxes. We chose the procedures for coccolithophores and radiolarians for this paper.

For coccolithophores and coccoliths, generally, an aliquot size of $1 / 4,096$ (one of $4,096^{\text {th }}$ ) of a liquid sample containing particles was filtered though a Gelman ${ }^{\circledR}$ membrane filter and a funnel with a rectangular opening of $34 \times 16$ $\mathrm{mm}$ in size (Figs. 2-4). When a population of coccolithophores and coccoliths on a filter was determined to be too thick or too thin to be able to efficiently count the following alternative aliquot sizes were sometimes used: $1 / 1,024,1 / 256$, $1 / 16,384$ and $1 / 65,536$. The filtered sample was desalted with distilled water and dried in an oven at $50^{\circ} \mathrm{C}$ overnight, and then permanently mounted with Olympus immersion oil Type- $\mathrm{F}^{\circledR}$ on a microslide. The coccolithophores and coccoliths were counted on a compound light microscope, employing the magnification of $x 600$. The calcareous nannoplankton fluxes are expressed as number of coccolithophores. The following conversion for a certain number of placoliths into one coccolithophore was made with the following rule: one coccolithophore of $C$. pelagicus represents 14 placoliths; and one coccolithophore of $E$. huxleyi represents 22 placoliths. The objective of the conversion is to understand accurate coccolithophore fluxes. Such conversions are applied only to the C. pelagicus and E. huxleyi cases, based on the observation of ca. twenty ccoccolithophore specimens for each taxon (Tsutsui et al., submitted).

For radiolarian enumerations, a $1 / 4$ aliquot size sample was split into aliquot sizes of $1 / 16$ to $1 / 1,024$. A split sample was sieved through a stainless steel screen with $63 \mu \mathrm{m}$ mesh first and then filtered through Gelman ${ }^{\circledR}$ membrane filters with a nominal pore size of $0.45 \mu \mathrm{m}$ for retaining all the particles for quantitative counts. The filtered sample was desalted with distilled water and dried in an oven at $50^{\circ} \mathrm{C}$ overnight, and then permanently mounted with Canada Balsam ${ }^{\circledR}$ on microslides. Each sample of a rectangular shape with $34.7 \times 16.1 \mathrm{~mm}$ in size

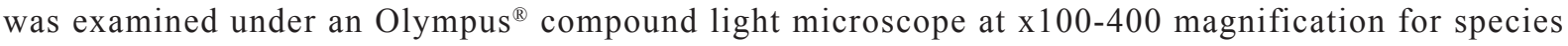
identification and counting (Ikenoue et al., 2012).

\subsection{Examples of long-term fluxes with total mass, biogenic opal, calcium carbonate, Others, and calcareous nannoplankton}

It is of interest to illustrate examples of long-term time-series particle fluxes. The obtained 19 year long timeseries fluxes of total mass, biogenic opal, calcium carbonate and Others are shown (Figs. 5-6) (Takahashi et al., 2011). Among many available data on the time-series fluxes, we have chosen calcareous nannoplankton to demonstrate because that we have up to date flux information for the entire 19 year long data at Stations SA and $\mathrm{AB}$ (Figs. 7-8). The upper illustrations of Figs. 7-8 show the fluxes whereas the lower illustrations show percentages of taxa, at Stations $\mathrm{SA}$ and $\mathrm{AB}$, respectively. At the both sediment trap stations, two major taxa of calcareous nannoplankton are encountered. One is Coccolithus pelagicus and the other is Emiliania huxleyi (Figs. 7-8). Other taxa such as Gephyrocapsa oceanica, Umbilicosphaera sibogae, and Brrarudosphaera bigelowi were also encountered with minor occurrences compared to those of the dominant taxa. The mean flux of coccospheres at Station AB is $115.6 \times 10^{6}$ coccospheres $\mathrm{m}^{-2} \mathrm{~d}^{-1}$ and the Station SA is $91.1 \times 10^{6}$ coccospheres $\mathrm{m}^{-2} \mathrm{~d}^{-1}$. The contribution of C. pelagicus is ca. $55 \%$ in total flux, and that of E. huxleyi is ca. $42 \%$ at both trap stations. The percentage of E. huxleyi at Station SA contributes approximately 50\% during 1990 to 1999 . Furthermore, during 2000 to 2009, Coccolithus pelagicus holds high percentages at Station SA (Tsutsui et al., submitted). 
Kozo Takahashi, Hirofumi Asahi, Yusuke Okazaki, Jonaotaro Onodera, Hideto Tsutsui,

Takahito Ikenoue, Yoshiyuki Kanematsu, Seiji Tanaka and Shinya Iwasaki

Year

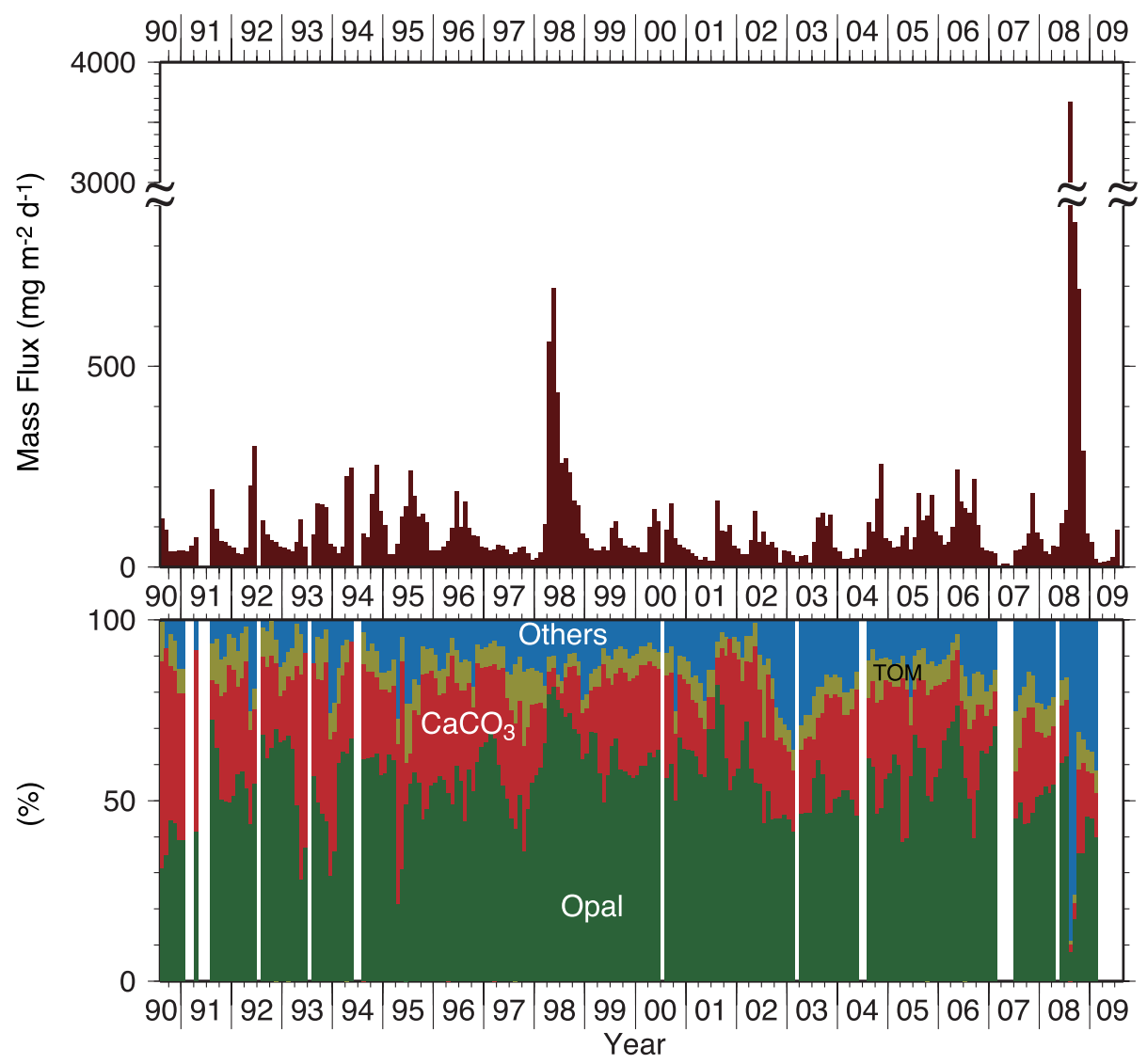

Fig. 5. Time-series fluxes of total mass and \% representations of biogenic opal, $\mathrm{CaCO}_{3}$, organic matter, and Others observed at Station SA during 1990-2009.

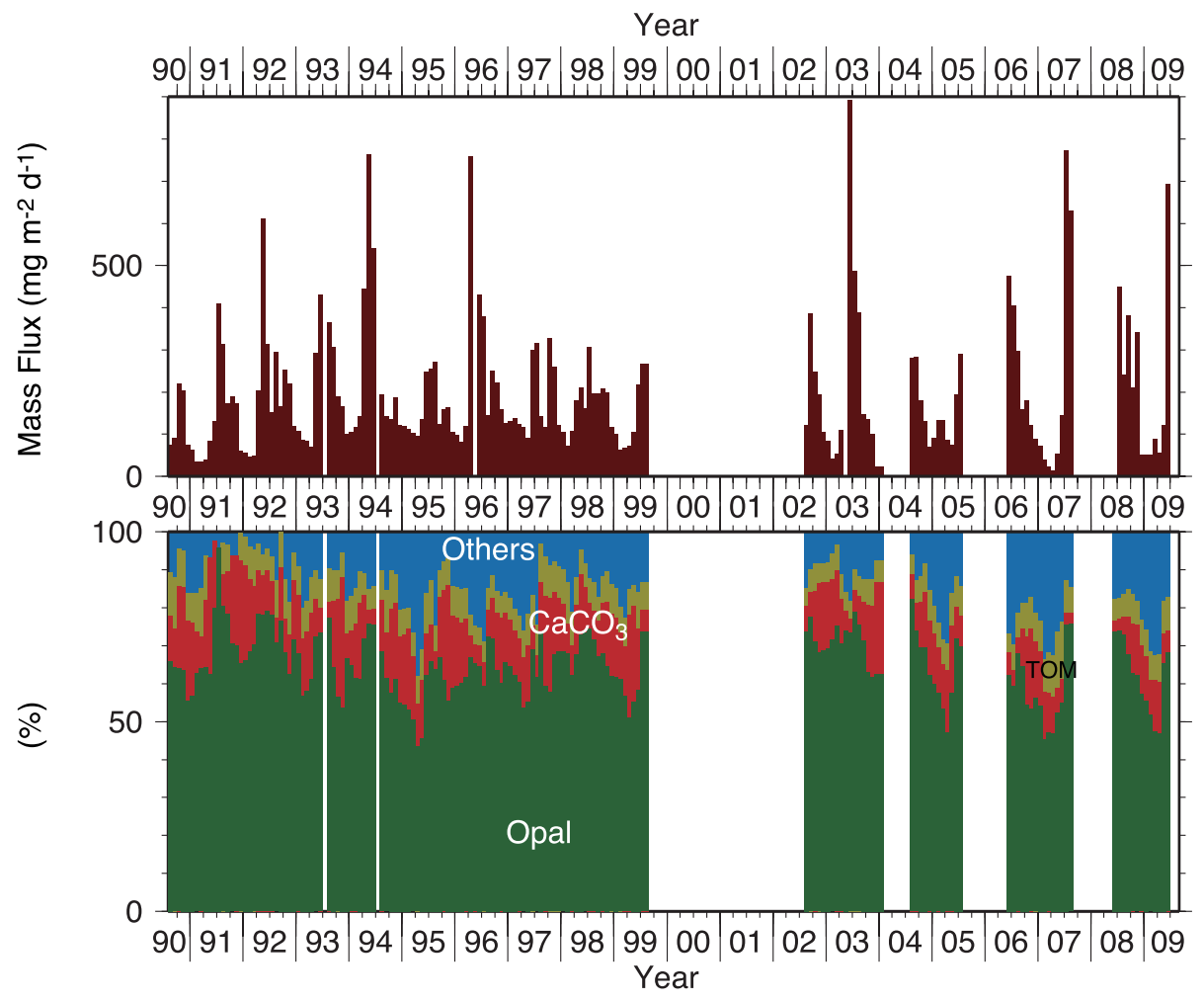

Fig. 6. Time-series fluxes of total mass and \% representations of biogenic opal, $\mathrm{CaCO}_{3}$, organic matter, and Others observed at Station AB during 1990-2009. Note that parts of the samples for 1999-2002, 2004, 2005-2006, 2007-2008, and 2009-2010 (shown with blank space) are missing due to either loss of the deployed mooring or malfunction of the traps (For details see Tables 1,3). 


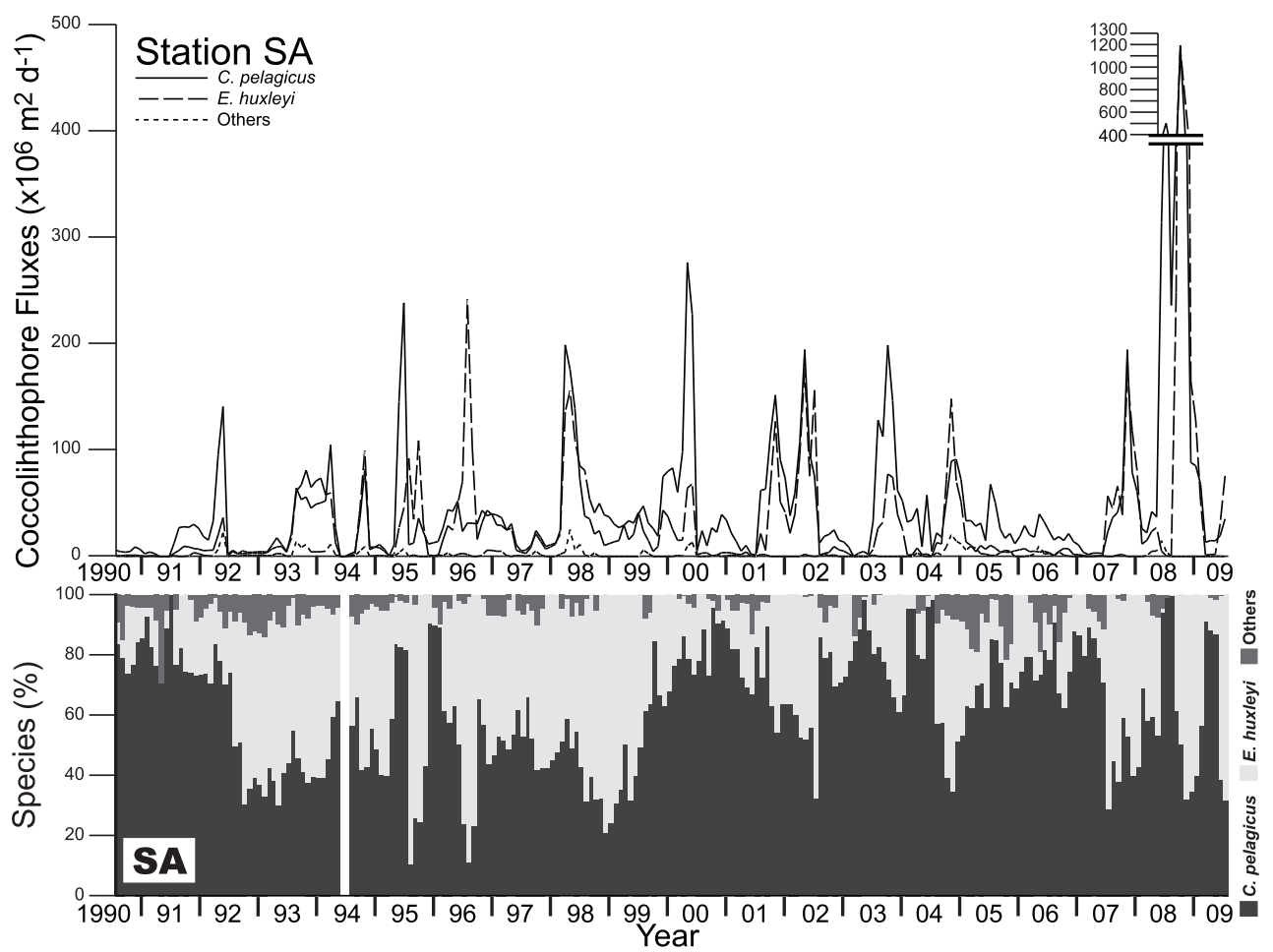

Fig. 7. Time-series fluxes of coccolithophores and species percentages of Coccolithus pelagicus and Emiliniania huxleyi at Station SA during 1990-2009.

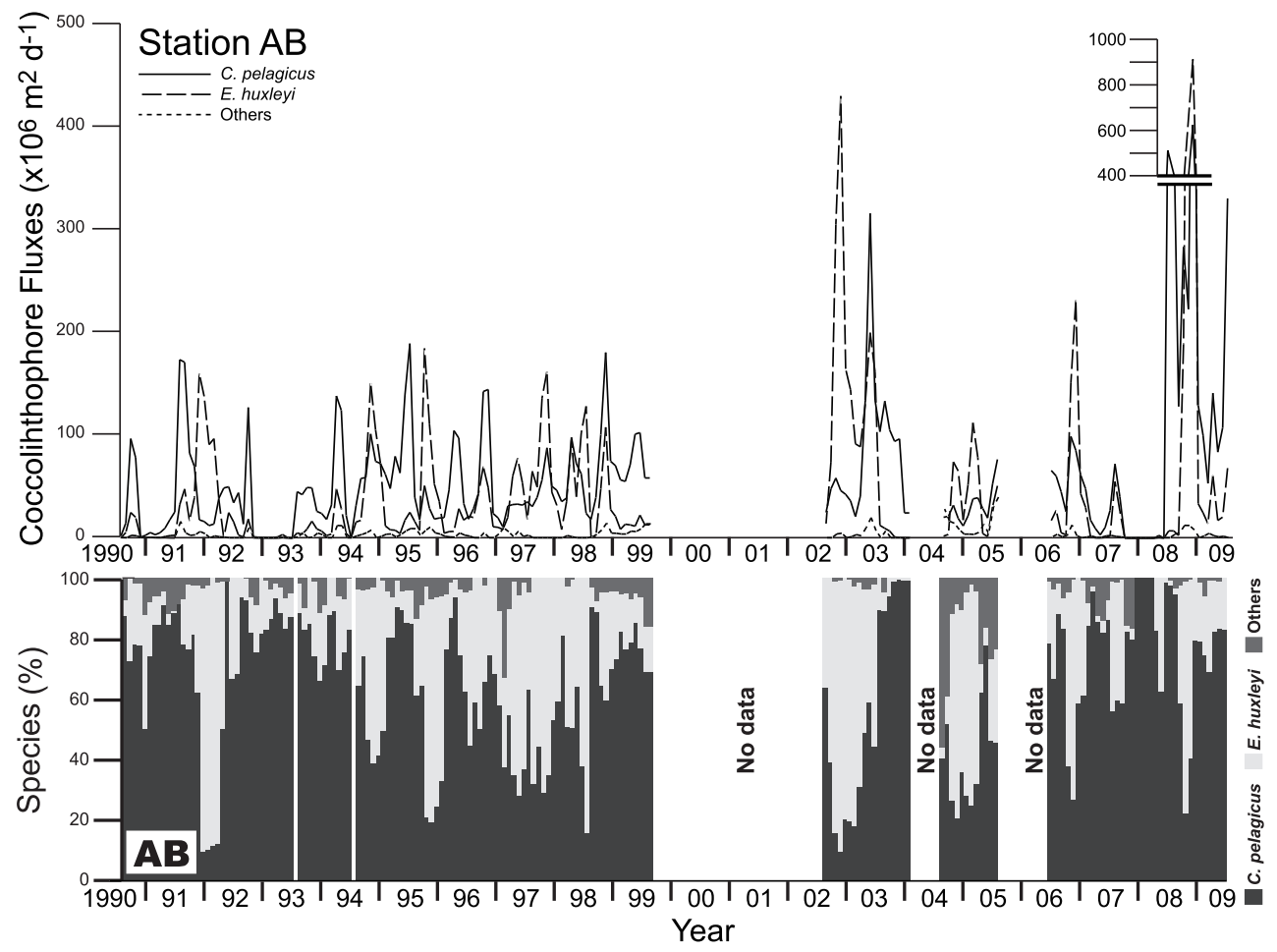

Fig. 8. Time-series fluxes of coccolithophores and species percentages of Coccolithus pelagicus and Emiliniania huxleyi at Station AB during 1990-2009. 


\section{Archival samples}

The trap samples have been processed as: (1) dried membrane filters (Figs. 2, 4); and (2) microslide samples (Figs. 3-4). The curatorial label is directly written in the empty area of a filter (Fig. 2); and a label of a microslide is given with a computer produced printout for legibility (Fig. 3). Examples of labels are illustrated (Fig. 4). The left side of Fig. 4 shows an example of sample identification information with weather resistance oil-based ink for a filtered sample. The right part illustrates a labeling for a microslide.

The valuable archived sediment trap samples, including those in liquid, have thus far been curated in refrigerators at the Laboratory of Paleoenvironmental Science at the Kyushu University. However, it is only possible for the museum to archive dried samples. The archives of the trap samples now have been officially transferred to the Kyushu University Museum we hereby describe the curatorial information of the archived samples with the specific data in the spreadsheets in Microsoft ${ }^{\circledR}$ Excel $^{\mathrm{TM}}$ format (Tables 4-7). The spreadsheets contain the following pieces of information relevant to the trap samples. The information concerning station identification, the deployment and recovery dates, geographic coordinates, water and trap depths are summarized (Table 1). The beginning and ending of each sampled intervals are given in two tables: Table 2 for Station SA and Table 3 for Station AB. The information concerning the museum archival samples are given in Table 4 (Station $\mathrm{SA}$ ) and Table 5 (Station AB) for membrane filters and in Table 6 (Station SA) and Table 7 (Station AB) for microslides.

A total number of the sample membrane filters listed herein is 973 for Station SA (Table 4) and 1,034 for Station AB (Table 5), respectively. Furthermore, the microslides prepared thus far and listed herein sum up to a total number of 835 for Station SA (Table 6) and 710 for Station AB (Table 7), respectively. Therefore, a grand total of the two types of samples listed in this paper extends to 3,552.

While the details of the samples given here represent a part of the archival effort, which is currently proceeding at the Kyushu University. Therefore, additional samples will be added to the lists in the future. Updated information concerning the sample lists will be provided by the planned electronic publications by the Kyusyu University Museum.

\section{Summary}

Description of the long-term time-series PARLUX type sediment trap samples are given in this paper so that the readers to understand the basics of the samples. Briefly, the sediment traps were moored at $600 \mathrm{~m}$ above the sea-floor at Station SA (water depth: 5,460 m) in the pelagic central subarctic Pacific and at hemipelagic Station $\mathrm{AB}$ (water depth: 3,788 m) in the Aleutian Basin of the Bering Sea. The time-series flux samples were obtained during 1990-2010 for nineteen years. Because that the time-series samples of the past can never be obtained again the archives of the existing samples are quite valuable for future studies. They can, for instance, be compared to those may be obtained in the future in the same region and elsewhere. This is particularly significant in the eyes of global warming which is taking place now (e.g., IPCC AR4 SYR, 2007).

The archived sediment trap samples are located at the Kyusyu University Museum for permanent preservation. The details of the samples are given herein and spreadsheet tables of the archives will also be electronically published by the Kyusyu University Museum. This allows future scientists and students, who need to examine the sediment trap samples, to be able to access the samples.

\section{Acknowledgements}

We sincerely thank funding provided by US NSF, MEXT, and JSPS for time-series collection of sediment trap samples at Stations SA and AB during 1989-2010. Regarding this archival effort of the trap samples, specific funds were provided by the Kyusyu University Museum for the past three years, for which we gratefully acknowledge. We thank Professor Emeriti Yoshiaki Maita, Mitsuru Yanada, Hideo Miyake and Dr. Hiroji Onishi of the Hokkaido University for jointly conducting the time-series trap experiment together at Stations SA and AB for such a long period of time as greater than two decades. We also thank other people, whose names are too numerous to mention here, but those who worked on the trap materials as graduate and undergraduate students under the supervision of the senior author, and Captains, officers, crew of T/S Oshoro-maru during the 22 summer cruises of 1989-2010 are acknowledged. A manuscript of this paper was reviewed and benefitted by Dr. Yoshihiro Nakamuta of the Kyusyu University Museum, for which we thank. 


\section{References}

Akagi, T., Fu, F. F., Hongo, Y., and Takahashi, K. (2011) Composition of rare earth elements in settling particles collected in the highly productive North Pacific Ocean and Bering Sea: implications for siliceous-matter dissolution kinetics and formation of two REE-enriched phases. Geochimica et Cosmochimica Acta, in press.

Asahi, H., and Takahashi, K. (2003) Multivariate analyses of planktonic foraminifera from the Bering Sea: a comparative study between the past and the present. Kaiyo Monthly, 35, 420-428. [In Japanese]

Asahi, H., Takahashi, K., and Yanada, M. (2007) Relationships between long time-series seasonal and inter-annual particle fluxes and climate change. Kaiyo Monthly, 39(2), 88-96. [In Japanese]

Asahi, H., and Takahashi, K. (2007) A 9-year time-series of planktonic foraminfer fluxes and environmental change in the Bering sea and the central subarctic Pacific Ocean, 1990-1999. Progress in Oceanography, 72, 343-363.

Asahi, H., and Takahashi, K. (2008) A new insight into oceanography with multivariate and time-series analyses on the 1990-1999 planktonic foraminiferal fluxes in the Bering Sea and the central subarctic Pacific. Memoirs of the Faculty of Sciences, Kyushu University, Series D, Earth and Planetary Sciences, 32(1), 73-96.

Ewing, M., and Connary, S. (1970) Nepheloid layer in the North Pacific. In: J.D. Hays, Editor, Geological investigations of the North Pacific, Geol. Soc. Am. Memoir, 126, 41-82.

Hashimoto, S., Maita, Y., Yanada, M., and Takahashi, K. (1997) Hexosamine contents in sinking particles as an indicator for biological processes in the Bering Sea and the central subarctic Pacific. In: Biogeochemical Processes in the North Pacific, Proc. Int. Mar. Sci. Symp., Mutsu, Japan, 1996. S. Tsunogai, Ed., Japan Marine Science Foundation, 290-299.

Hashimoto, S., Maita, Y., Yanada, M., and Takahashi, K. (1998) Annual and seasonal variations of amino acid and hexosamine fluxes in the deep Bering Sea and the deep central subarctic Pacific. Deep-Sea Res., 45(7), 1029-1051.

Honjo, S., and Doherty, K.W. (1988) Large aperture time-series sediment traps; design objectives, construction and application. Deep-Sea Res., 35, 133-149.

Honjo, S., Manganini, S.J., Krishfield, R.A., and Francois, R. (2008) Particulate organic carbon fluxes to the ocean interior and factors controlling the biological pump: A synthesis of global sediment trap programs since 1983. Progress in Oceanography, 76, 217-285.

Ikenoue, T., Takahashi, K., and Tanaka, S. (2012) Fifteen year time-series of radiolarian fluxes and environmental conditions in the Bering Sea and the central subarctic Pacific, 1990-2005. Deep-Sea Res. II, in press.

IPCC AR4 SYR (2007) Core Writing Team. In: Climate Change 2007: Synthesis Report, Pachauri, R.K., and Reisinger, A., Eds., Contribution of Working Groups I, II and III to the Fourth Assessment Report of the Intergovernmental Panel on Climate Change, IPCC, ISBN 92-9169-122-4.

Itaki, T., and Takahashi, K. (1995) Preliminary results on radiolarian fluxes in the central subarctic Pacific and Bering Sea. Proc. Hokkaido Tokai University Science and Engineering, 7(1994), 37-47. [In Japanese with English Abstract]

Itaki, T., Takahashi, K., and Maita, Y. (1997) Seasonal change of Cycladophora davisiana (Radiolaria): its significance for paleoceanography. News of Osaka Micropaleontologists, Special Volume, No. 10, 293-298. [In Japanese with English Abstract]

Kurihara, M., and Takahashi, K. (2002) Long-term size variation and life cycle patterns of a predominant diatom Neodenticula seminae in the subarctic Pacific and the Bering Sea. Bulletin of the Planktonic Society of Japan, 49(2), 76-86. [In Japanese with English Abstract]

Maita, Y., Yanada, M., Hashimoto, S., and Takahashi, K. (1996) Biological production processes assessed from organic compounds in the particle fluxes in the subarctic Pacific. Kaiyo Monthly, Spec. vol. No. 11, 105-110. [In Japanese]

Maita, Y., Yanada, M., and Takahashi, K. (1999) Seasonal variation in the process of marine organism production based on downward fluxes of organic substances in the Bering Sea. In: Dynamics of the Bering Sea, Loughlin, T.R., and K. Ohtani, Eds., University of Alaska Sea Grant, Fairbanks, Alaska, 341-352.

Ohtani, K. (1965) On the Alaskan Stream in summer. Bull. Fac. Fish., Hokkaido Univ., 15: 260-273. [In Japanese]

Onodera, J., Takahashi, K., and Yanada, M. (2007) Diatom time-series fluxes at Stations AB, SA. Kaiyo Monthly, 39(2), 97-103. [In Japanese]

Onodera, J., and Takahashi, K. (2009) Long-term diatom fluxes in response to oceanographic conditions at Stations AB and SA in the central subarctic Pacific and the Bering Sea, 1990-1998. Deep-Sea Research I, 56(2), 189-211.

Onodera, J., Takahashi, K., Onishi, H., and Yanada, M. (2009) Diatom floral fluxes at Stations AG and SA in the Bering Sea and the northern Subarctic Pacific, 1990-1998. Umi no Kenkyu, Oceanogr. Soc. Japan, 18, 307-322. [In Japanese with English Abstract]

Onodera, J., and Takahashi, K. (2011) Oceanographic conditions influencing silicoflagellate assemblages in the Bering Sea and subarctic Pacific Ocean during 1990-1994. Deep-Sea Res. II, in press. 
Reid, P.C., Johns, D.G., Edwards, M., Starr, M., Poulin, M., and Snoeijs, P. (2007). A biological consequence of reducing Arctic ice cover: arrival of the Pacific diatom Neodenticula seminae in the North Atlantic for the first time in 800000 years. Global Change Biology, 13, 1910-1921.

Stabeno, P.J., Schumacher, J.D., and Ohtani, K. (1999) The physical oceanography of the Ber- ing Sea. In: Dynamics of the Bering Sea: A Summary of Physical, Chemical, and Biological Characteristics, and a Synopsis of Research on the Bering Sea, Loughlin, T.R., and Ohtani, K., Eds., Fairbanks (Univ. Alaska Sea Grant), 1-28.

Takahashi, K. (1986) Seasonal fluxes of pelagic diatoms in the subarctic Pacific, 1982-1983. Deep-Sea Research, 33 : 1225-1251.

Takahashi, K. (1987a) Seasonal Fluxes of Silicoflagellates and Actiniscus in the Subarctic Pacific During 1982-1984, Jour. Mar. Res., 45: 397-425.

Takahashi, K. (1987b) Response of subarctic Pacific diatom fluxes to the 1982-1983 El Nino. Jour. Geophys. Res., 92 (13): 14,387-14,392.

Takahashi, K. (1987c) Radiolarian Flux and Seasonality: Climatic and El Nino Response in the Subarctic Pacific, $1982-$ 1984. Global Biogeochemical Cycles, 1 (3): 213-231.

Takahashi, K. (1991) Radiolaria: flux, ecology, and taxonomy in the Pacific and Atlantic. In: Ocean Biocoenosis, Ed., Honjo, S., Series No. 3, Woods Hole Oceanographic Institution Press, 303 pp.

Takahashi, K. (1994) From modern flux to paleoflux: assessment from sinking assemblages to thanatocoenosis. In: Carbon Cycling in the Glacial Ocean: Constrains on the Ocean's Role in Global Change, R. Zahn, et al., Eds., NATO ASI Series, Vol. 117, 413-424.

Takahashi, K. (1995) Opal particle flux in the subarctic Pacific and Bering Sea and sidocoenosis preservation hypothesis. In: Global fluxes of carbon and its related substances in the coastal sea-ocean-atmosphere system, Proceedings of the 1994 Sapporo IGBP Symposium, S. Tsunogai et al., Eds., M \& J. International, Yokohama, Japan. 458-466.

Takahashi, K. (1999) Paleoceanographic changes and present environment of the Bering Sea. In: Dynamics of the Bering Sea, Loughlin, T.R., and K. Ohtani, Eds., University of Alaska Sea Grant, Fairbanks, Alaska, 365-385.

Takahashi, K. (2005) The Bering Sea and paleoceanography. Deep-Sea Res. II, 52 (16/18), 2080-2091.

Takahashi, K., Hurd, D. C., and Honjo, S. (1983) Phaeodarian skeletons: Their role in silica transport to the deep sea. Science, 222(4624): 616-618.

Takahashi, K., Honjo, S., and Tabata, S. (1989) Siliceous phytoplankton flux: interannual variability and response to hydrographic changes in the northeastern Pacific. In: Aspects of Climate Variability in the Pacific and Western Americas, Ed., D. Peterson, Geophysical Monograph 55, Amer. Geophys. Union, 151-160.

Takahashi, K., Billings, J. D., and Morgan, J. K. (1990) Oceanic province: assessment from the time-series diatom production in the northeastern Pacific. Limnol. Oceanogr., 35 (1), 154-165.

Takahashi, K., Hisamichi, K., Yanada, M., and Maita, Y. (1996) Seasonal change of phytoplankton productivity: results from sediment traps. Kaiyo Monthly, Special volume, No. 10, 109-115. [In Japanese with English fig. \& table captions]

Takahashi, K., Fujitani, N., Yanada, M., and Maita, Y. (1997) Five year long particle fluxes in the central subarctic Pacific and the Bering Sea. In: Biogeochemical Processes in the North Pacific, Proc. Int. Mar. Sci. Symp., Mutsu, Japan, 1996. S. Tsunogai, Ed., Japan Marine Science Foundation, 277-289.

Takahashi, K., Fujitani, N., Yanada, M., and Maita, Y. (2000) Long-term biogenic particle fluxes in the Bering Sea and the central subarctic Pacific Ocean, 1990-1995. Deep-Sea Res. I, 47 (9), 1723-1759.

Takahashi, K., Fujitani, N., and Yanada, M. (2002) Long term monitoring of particle fluxes in the Bering Sea and the central subarctic Pacific Ocean, 1990-2000. Progress in Oceanography, 55 (1-2), 95-112.

Takahashi, K., Yanada, M., Onodera, J., and Kanematsu, Y. (2007) Long time-series flux observation in the Bering Sea and the North Pacific, 1990-2006: Current status and future direction. Kaiyo Monthly, 39(2), 80-87. [In Japanese]

Takahashi, K., Kanematsu, Y., Asahi, H., Onodera, J., Okazaki, Y., Tanaka, S., and Tsutsui, H. (2011) Biological response to the global climate regime shift in the Bering Sea and the central subarctic Pacific: Synthesis of multidecadal long time series sinking particle study. Abstract PP42A-04 presented at 2011 Fall Meeting, AGU, San Francisco, Calif., 5-9 Dec.

Tsutsui, H., Takahashi, K., Asahi, H., Nishida, S., Nishiwaki, N., and Yamamoto, S. Nineteen-year long time-series fluxes of calcareous nannoplankton in the Bering Sea and the central subarctic Pacific Ocean, 1990-2009. Deep-Sea Res. II, submitted. 
Table 2. Sample dates and intervals of the sediment trap materials recovered from Stations SA, 1990-2009.

\begin{tabular}{|c|c|c|c|c|c|c|c|c|c|}
\hline Sequential \# & Sample \# & Open Date & Closed Date & Interval (d) & Sequential \# & Sample \# & Open Date & Closed Date & Interval (d) \\
\hline \multicolumn{5}{|l|}{ YEAR 1} & \multicolumn{5}{|l|}{ YEAR 5} \\
\hline 1 & SA2\#1 & 09Aug1990 & 23Aug1990 & 14 & 53 & SA6\#1 & 06Aug1994 & 23Aug1994 & 17 \\
\hline 2 & $\mathrm{SA} 2 \# 2$ & 23Aug1990 & 12Sep1990 & 20 & 54 & SA6\#2 & 23Aug1994 & 12Sep1994 & 20 \\
\hline 3 & $\mathrm{SA} 2 \# 3$ & 12Sep1990 & 02Oct1990 & 20 & 55 & SA6\#3 & 12Sep1994 & $02 \mathrm{Oct} 1994$ & 20 \\
\hline 4 & SA2\#4 & 02Oct1990 & 27Nov1990 & 56 & 56 & SA6\#4 & 02 Oct1994 & 22Oct1994 & 20 \\
\hline 5 & $\mathrm{SA} 2 \# 5$ & 27Nov1990 & 22Jan1991 & 56 & 57 & SA6\#5 & 22Oct1994 & 27Nov1994 & 36 \\
\hline 6 & SA2\#6 & 22Jan1991 & 19Mar1991 & 56 & 58 & SA6\#6 & 27Nov1994 & 22Jan1995 & 56 \\
\hline 7 & SA2\#7 & 19Mar1991 & 08Apr1991 & 20 & 59 & SA6\#7 & 22Jan1995 & 19Mar1995 & 56 \\
\hline 8 & SA2\#8 & 08Apr1991 & 28Apr1991 & 20 & 60 & SA6\#8 & 19Mar1995 & 08Apr1995 & 20 \\
\hline 9 & SA2\#9 & 28Apr1991 & 18May1991 & 20 & 61 & SA6\#9 & 08Apr1995 & 28Apr1995 & 20 \\
\hline 10 & SA2\#10 & 18May1991 & 07Jun1991 & 20 & 62 & SA6\#10 & 28Apr1995 & 13May1995 & 15 \\
\hline 11 & SA2\#11 & 07Jun1991 & 27Jun1991 & 20 & 63 & SA6\#11 & 13May 1995 & 28May 1995 & 15 \\
\hline 12 & SA2\#12 & 27Jun1991 & 17Jul1991 & 20 & 64 & SA6\#12 & 28May 1995 & 17Jun1995 & 20 \\
\hline 13 & SA2\#13 & 17Jul1991 & 04Aug1991 & 18 & 65 & SA6\#13 & 17Jun1995 & 02Aug1995 & 46 \\
\hline \multicolumn{5}{|l|}{ YEAR 2} & \multicolumn{5}{|l|}{ YEAR 6} \\
\hline 14 & SA3\#1 & 11Aug1991 & 23Aug1991 & 12 & 66 & SA7\#1 & 10Aug1995 & 12Sep1995 & 33 \\
\hline 15 & $\mathrm{SA} 3 \# 2$ & 23Aug1991 & 12Sep1991 & 20 & 67 & SA7\#2 & 12Sep1995 & $02 \mathrm{Oct} 1995$ & 20 \\
\hline 16 & SA3\#3 & 12Sep1991 & 02Oct1991 & 20 & 68 & SA7\#3 & 02 Oct1995 & $22 \mathrm{Oct} 1995$ & 20 \\
\hline 17 & SA3\#4 & 02Oct1991 & 27Nov1991 & 56 & 69 & SA7\#4 & 22Oct1995 & 27Nov1995 & 36 \\
\hline 18 & SA3\#5 & 27Nov1991 & 22Jan1992 & 56 & 70 & SA7\#5 & 27Nov1995 & 22Jan1996 & 56 \\
\hline 19 & SA3\#6 & 22Jan1992 & 19Mar1992 & 57 & 71 & SA7\#6 & 22Jan1996 & 19Mar1996 & 57 \\
\hline 20 & SA3\#7 & 19Mar1992 & 08Apr1992 & 20 & 72 & SA7\#7 & 19Mar1996 & 08Apr1996 & 20 \\
\hline 21 & SA3\#8 & 08Apr1992 & 28Apr1992 & 20 & 73 & SA7\#8 & 08Apr1996 & 28Apr1996 & 20 \\
\hline 22 & SA3\#9 & 28Apr1992 & 18May 1992 & 20 & 74 & SA7\#9 & 28Apr1996 & 18May1996 & 20 \\
\hline 23 & SA3\#10 & 18May1992 & 07Jun1992 & 20 & 75 & SA7\#10 & 18May1996 & 07Jun1996 & 20 \\
\hline 24 & SA3\#11 & 07Jun1992 & 27Jun1992 & 20 & 76 & SA7\#11 & 07Jun1996 & 27Jun1996 & 20 \\
\hline 25 & SA3\#12 & 27Jun1992 & 17Jul1992 & 20 & 77 & SA7\#12 & 27Jun1996 & 17Jul1996 & 20 \\
\hline 26 & SA3\#13 & 17Jul1992 & 02Aug1992 & 16 & 78 & SA7\#13 & 17Jul1996 & 02Aug1996 & 16 \\
\hline \multicolumn{5}{|l|}{ YEAR 3} & \multicolumn{5}{|c|}{ YEAR 7} \\
\hline 27 & SA4\#1 & 10Aug1992 & 23Aug1992 & 13 & 79 & SA8\#1 & 11Aug1996 & 23Aug1996 & 12 \\
\hline 28 & $\mathrm{SA} 4 \# 2$ & 23Aug1992 & 12Sep1992 & 20 & 80 & SA8\#2 & 23Aug1996 & 12Sep1996 & 20 \\
\hline 29 & SA4\#3 & 12Sep1992 & $02 \mathrm{Oct} 1992$ & 20 & 81 & SA8\#3 & 12Sep1996 & $02 \mathrm{Oct} 1996$ & 20 \\
\hline 30 & SA4\#4 & 02Oct1992 & 22Oct1992 & 20 & 82 & SA8\#4 & $02 \mathrm{Oct} 1996$ & $22 \mathrm{Oct} 1996$ & 20 \\
\hline 31 & SA4\#5 & 22Oct1992 & 27Nov1992 & 36 & 83 & SA8\#5 & $22 \mathrm{Oct} 1996$ & 27Nov1996 & 36 \\
\hline 32 & SA4\#6 & 27Nov1992 & 22Jan1993 & 56 & 84 & SA8\#6 & 27Nov1996 & 22Jan1997 & 56 \\
\hline 33 & SA4\#7 & 22Jan1993 & 19Mar1993 & 56 & 85 & SA8\#7 & 22Jan1997 & 19Mar1997 & 56 \\
\hline 34 & SA4\#8 & 19Mar1993 & 08Apr1993 & 20 & 86 & SA8\#8 & 19Mar1997 & 08Apr1997 & 20 \\
\hline 35 & SA4\#9 & 08Apr1993 & 28Apr1993 & 20 & 87 & SA8\#9 & 08Apr1997 & 28Apr1997 & 20 \\
\hline 36 & SA4\#10 & 28Apr1993 & 18May1993 & 20 & 88 & SA8\#10 & 28Apr1997 & 18May1997 & 20 \\
\hline 37 & SA4\#11 & 18May 1993 & 07Jun1993 & 20 & 89 & SA8\#11 & 18May1997 & 07Jun1997 & 20 \\
\hline 38 & SA4\#12 & 07Jun1993 & 27Jun1993 & 20 & 90 & SA8\#12 & 07Jun1997 & 27Jun1997 & 20 \\
\hline 39 & SA4\#13 & 27Jun1993 & 20Jul1993 & 23 & 91 & SA8\#13 & 27Jun1997 & 04Aug1997 & 38 \\
\hline \multicolumn{5}{|l|}{ YEAR 4} & \multicolumn{5}{|l|}{ YEAR 8} \\
\hline 40 & SA5\#1 & 10Aug1993 & 23Aug1993 & 13 & 92 & SA9\#1 & 10Aug1997 & 23Aug1997 & 13 \\
\hline 41 & SA5\#2 & 23Aug1993 & 12Sep1993 & 20 & 93 & SA9\#2 & 23Aug1997 & 12Sep1997 & 20 \\
\hline 42 & SA5\#3 & 12Sep1993 & $02 \mathrm{Oct} 1993$ & 20 & 94 & SA9\#3 & 12Sep1997 & $02 \mathrm{Oct} 1997$ & 20 \\
\hline 43 & SA5\#4 & 02 Oct1993 & $22 \mathrm{Oct} 1993$ & 20 & 95 & SA9\#4 & 02 Oct1997 & $22 \mathrm{Oct} 1997$ & 20 \\
\hline 44 & SA5\#5 & $22 \mathrm{Oct} 1993$ & 27Nov1993 & 36 & 96 & SA9\#5 & $22 \mathrm{Oct} 1997$ & 27Nov1997 & 36 \\
\hline 45 & SA5\#6 & 27Nov1993 & 22Jan1994 & 56 & 97 & SA9\#6 & 27Nov1997 & 22Jan1998 & 56 \\
\hline 46 & SA5\#7 & 22Jan1994 & 19Mar1994 & 56 & 98 & SA9\#7 & 22Jan1998 & 19Mar1998 & 56 \\
\hline 47 & SA5\#8 & 19Mar1994 & 08Apr1994 & 20 & 99 & SA9\#8 & 19Mar1998 & 08Apr1998 & 20 \\
\hline 48 & SA5\#9 & 08Apr1994 & 28Apr1994 & 20 & 100 & SA9\#9 & 08Apr1998 & 28Apr1998 & 20 \\
\hline 49 & SA5\#10 & 28Apr1994 & 18May1994 & 20 & 101 & SA9\#10 & 28Apr1998 & 18May1998 & 20 \\
\hline 50 & SA5\#11 & 18May 1994 & 07Jun1994 & 20 & 102 & SA9\#11 & 18May 1998 & 07Jun1998 & 20 \\
\hline 51 & SA5\#12 & 07Jun1994 & 27Jun1994 & 20 & 103 & SA9\#12 & 07Jun1998 & 27Jun1998 & 20 \\
\hline 52 & SA5\#13 & 27Jun1994 & 02Aug1994 & 36 & 104 & SA9\#13 & 27Jun 1998 & 05Aug1998 & 39 \\
\hline
\end{tabular}


Kozo Takahashi, Hirofumi Asahi, Yusuke Okazaki, Jonaotaro Onodera, Hideto Tsutsui,

Takahito Ikenoue, Yoshiyuki Kanematsu, Seiji Tanaka and Shinya Iwasaki

Table 2 (Cont.)

\begin{tabular}{|c|c|c|c|c|c|c|c|c|c|}
\hline Sequential \# & Sample \# & Open Date & Closed Date & Interval (d) & Sequential \# & Sample \# & Open Date & Closed Date & Interval (d) \\
\hline YEAR 9 & & & & & 158 & SA14\#2 & 23Aug2002 & 12Sep2002 & 20 \\
\hline 105 & SA10\#1 & 10Aug1998 & 23Aug1998 & 13 & 159 & SA14\#3 & 12Sep2002 & $02 \mathrm{Oct} 2002$ & 20 \\
\hline 106 & SA10\#2 & 23Aug1998 & 12Sep1998 & 20 & 160 & SA14\#4 & $02 \mathrm{Oct} 2002$ & $22 \mathrm{Oct} 2002$ & 20 \\
\hline 107 & SA10\#3 & 12Sep1998 & 02 Oct1998 & 20 & 161 & SA14\#5 & $22 \mathrm{Oct} 2002$ & 27 Nov2002 & 36 \\
\hline 108 & SA10\#4 & $02 \mathrm{Oct} 1998$ & $22 \mathrm{Oct} 1998$ & 20 & 162 & SA14\#6 & 27Nov2002 & 22Jan2003 & 56 \\
\hline 109 & SA10\#5 & $22 \mathrm{Oct} 1998$ & 27Nov1998 & 36 & 163 & SA14\#7 & 22Jan2003 & 28Feb2003 & 37 \\
\hline 110 & SA10\#6 & 27Nov1998 & 22Jan1999 & 56 & 164 & SA14\#8 & $28 \mathrm{Feb} 2003$ & 08Apr2003 & 39 \\
\hline 111 & SA10\#7 & 22Jan1999 & 19Mar1999 & 56 & 165 & SA14\#9 & 08Apr2003 & 28Apr2003 & 20 \\
\hline 112 & SA10\#8 & 19Mar1999 & 08Apr1999 & 20 & 166 & SA14\#10 & 28Apr2003 & 18Мay2003 & 20 \\
\hline 113 & SA10\#9 & 08Apr1999 & 28Apr1999 & 20 & 167 & SA14\#11 & 18May2003 & 07Jun2003 & 20 \\
\hline 114 & SA10\#10 & 28Apr1999 & 18May1999 & 20 & 168 & SA14\#12 & 07Jun2003 & 27Jun2003 & 20 \\
\hline 115 & SA10\#11 & 18May 1999 & 07Jun1999 & 20 & 169 & SA14\#13 & 27Jun2003 & 10Jul2003 & 13 \\
\hline 116 & SA10\#12 & 07Jun1999 & 27Jun1999 & 20 & YEAR 14 & & & & \\
\hline 117 & SA10\#13 & 27Jun1999 & 05Aug1999 & 39 & 170 & SA15\#1 & 16Jul2003 & 03Aug2003 & 18 \\
\hline YEAR 10 & & & & & 171 & SA15\#2 & 03Aug2003 & 23Aug2003 & 20 \\
\hline 118 & SA11\#1 & 09Aug1999 & 23Aug1999 & 14 & 172 & SA15\#3 & 23Aug2003 & 12Sep2003 & 20 \\
\hline 119 & SA11\#2 & 23Aug1999 & 12Sep1999 & 20 & 173 & SA15\#4 & 12Sep2003 & $02 \mathrm{Oct} 2003$ & 20 \\
\hline 120 & SA11\#3 & 12Sep1999 & 02Oct1999 & 20 & 174 & SA15\#5 & $02 \mathrm{Oct} 2003$ & $22 \mathrm{Oct} 2003$ & 20 \\
\hline 121 & SA11\#4 & 02Oct1999 & 22Oct1999 & 20 & 175 & SA15\#6 & $22 \mathrm{Oct} 2003$ & 27 Nov2003 & 36 \\
\hline 122 & SA11\#5 & $22 \mathrm{Oct} 1999$ & 27Nov1999 & 36 & 176 & SA15\#7 & 27 Nov2003 & 22Jan2004 & 56 \\
\hline 123 & SA11\#6 & 27Nov1999 & 22Jan2000 & 56 & 177 & SA15\#8 & 22Jan2004 & 08Apr2004 & 77 \\
\hline 124 & SA11\#7 & 22Jan2000 & 18 Mar2000 & 56 & 178 & SA15\#9 & 08Apr2004 & 28Apr2004 & 20 \\
\hline 125 & SA11\#8 & $18 \operatorname{Mar} 2000$ & 07Apr2000 & 20 & 179 & SA15\#10 & 28Apr2004 & 18May2004 & 20 \\
\hline 126 & SA11\#9 & 07Apr2000 & 27Apr2000 & 20 & 180 & SA15\#11 & 18May2004 & 07Jun2004 & 20 \\
\hline 127 & SA11\#10 & $27 \mathrm{Apr} 2000$ & 17May2000 & 20 & 181 & SA15\#12 & 07Jun2004 & 27Jun2004 & 20 \\
\hline 128 & SA11\#11 & 17May2000 & 06Jun2000 & 20 & 182 & SA15\#13 & 27Jun2004 & 10Jul2004 & 13 \\
\hline 129 & SA11\#12 & 06Jun2000 & 26Jun2000 & 20 & YEAR 15 & & & & \\
\hline 130 & SA11\#13 & 26Jun2000 & 04Aug2000 & 39 & 183 & SA16\#1 & 12Aug2004 & 23Aug2004 & 11 \\
\hline YEAR 11 & & & & & 184 & SA16\#2 & 23Aug2004 & 12Sep2004 & 20 \\
\hline 131 & SA12\#1 & 09Aug2000 & 23Aug2000 & 14 & 185 & SA16\#3 & 12Sep2004 & $02 \mathrm{Oct} 2004$ & 20 \\
\hline 132 & SA12\#2 & 23Aug2000 & 12Sep2000 & 20 & 186 & SA16\#4 & 02Oct2004 & $22 \mathrm{Oct} 2004$ & 20 \\
\hline 133 & SA12\#3 & 12Sep2000 & 02Oct2000 & 20 & 187 & SA16\#5 & $22 \mathrm{Oct} 2004$ & 27Nov2004 & 36 \\
\hline 134 & SA12\#4 & 02Oct2000 & $22 \mathrm{Oct} 2000$ & 20 & 188 & SA16\#6 & 27 Nov2004 & 22Jan2005 & 56 \\
\hline 135 & SA12\#5 & $22 \mathrm{Oct} 2000$ & 27 Nov2 2000 & 36 & 189 & SA16\#7 & 22Jan2005 & 19Mar2005 & 56 \\
\hline 136 & SA12\#6 & 27 Nov 2000 & 22Jan2001 & 56 & 190 & SA16\#8 & 19Mar2005 & 08Apr2005 & 20 \\
\hline 137 & SA12\#7 & 22Jan2001 & 19Mar2001 & 56 & 191 & SA16\#9 & 08Apr2005 & 28Apr2005 & 20 \\
\hline 138 & SA12\#8 & 19Mar2001 & 08Apr2001 & 20 & 192 & SA16\#10 & 28Apr2005 & 18May2005 & 20 \\
\hline 139 & SA12\#9 & 08Apr2001 & 28Apr2001 & 20 & 193 & SA16\#11 & 18May2005 & 07Jun2005 & 20 \\
\hline 140 & SA12\#10 & 28Apr2001 & 18May2001 & 20 & 194 & SA16\#12 & 07Jun2005 & 27Jun2005 & 20 \\
\hline 141 & SA12\#11 & 18May2001 & 07Jun2001 & 20 & 195 & SA16\#13 & 27Jun2005 & 06Jul2005 & 9 \\
\hline 142 & SA12\#12 & 07Jun2001 & 27Jun2001 & 20 & YEAR 16 & & & & \\
\hline 143 & SA12\#13 & 27Jun2001 & 05Aug2001 & 39 & 196 & SA17\#1 & 10Jul2005 & 03Aug2005 & 24 \\
\hline YEAR 12 & & & & & 197 & SA17\#2 & 03Aug2005 & 23Aug2005 & 20 \\
\hline 144 & SA13\#1 & 07Aug2001 & 23Aug2001 & 16 & 198 & SA17\#3 & 23Aug2005 & 02Sep2005 & 10 \\
\hline 145 & SA13\#2 & 23Aug2001 & 12Sep2001 & 20 & 199 & SA17\#4 & 02Sep2005 & 12Sep2005 & 10 \\
\hline 146 & SA13\#3 & 12Sep2001 & $02 \mathrm{Oct} 2001$ & 20 & 200 & SA17\#5 & 12Sep2005 & 22Sep2005 & 10 \\
\hline 147 & SA13\#4 & 02Oct2001 & $22 \mathrm{Oct} 2001$ & 20 & 201 & SA17\#6 & 22Sep2005 & $02 \mathrm{Oct} 2005$ & 10 \\
\hline 148 & SA13\#5 & $22 \mathrm{Oct} 2001$ & 27Nov2001 & 36 & 202 & SA17\#7 & $02 \mathrm{Oct} 2005$ & $12 \mathrm{Oct} 2005$ & 10 \\
\hline 149 & SA13\#6 & 27 Nov2001 & 22Jan2002 & 56 & 203 & SA17\#8 & $12 \mathrm{Oct} 2005$ & $22 \mathrm{Oct} 2005$ & 10 \\
\hline 150 & SA13\#7 & 22Jan2002 & 19Mar2002 & 56 & 204 & SA17\#9 & $22 \mathrm{Oct} 2005$ & 09Nov2005 & 18 \\
\hline 151 & SA13\#8 & 19Mar2002 & 08Apr2002 & 20 & 205 & SA17\#10 & 09Nov2005 & 27Nov2005 & 18 \\
\hline 152 & SA13\#9 & 08Apr2002 & 28Apr2002 & 20 & 206 & SA17\#11 & 27 Nov2005 & 22Jan2006 & 56 \\
\hline 153 & SA13\#10 & 28Apr2002 & 18May2002 & 20 & 207 & SA17\#12 & 22Jan2006 & $28 \mathrm{Feb} 2006$ & 37 \\
\hline 154 & SA13\#11 & 18May2002 & 07Jun2002 & 20 & 208 & SA17\#13 & 28Feb2006 & 19Mar2006 & 19 \\
\hline 155 & SA13\#12 & 07Jun2002 & 27Jun2002 & 20 & 209 & SA17\#14 & 19Mar2006 & 29Mar2006 & 10 \\
\hline 156 & SA13\#13 & 27Jun2002 & 31Jul2002 & 34 & 210 & SA17\#15 & 29Mar2006 & 08Apr2006 & 10 \\
\hline YEAR 13 & & & & & 211 & SA17\#16 & 08Apr2006 & 18Apr2006 & 10 \\
\hline 157 & SA14\#1 & 18Aug2002 & 23Aug2002 & 5 & 212 & SA17\#17 & 18Apr2006 & 28Apr2006 & 10 \\
\hline
\end{tabular}


Table 2 (Cont.)

\begin{tabular}{|c|c|c|c|c|c|c|c|c|c|}
\hline Sequential \# & Sample \# & Open Date & Closed Date & Interval (d) & Sequential \# & Sample \# & Open Date & Closed Date & Interval (d) \\
\hline 213 & SA17\#18 & 28Apr2006 & 08Мау2006 & 10 & 249 & SA19\#12 & 22Jan2008 & 28Feb2008 & 37 \\
\hline 214 & SA17\#19 & 08May2006 & 18May2006 & 10 & 250 & SA19\#13 & $28 \mathrm{Feb} 2008$ & 19Mar2008 & 20 \\
\hline 215 & SA17\#20 & 18May2006 & 28May2006 & 10 & 251 & SA19\#14 & 19Mar2008 & 29Mar2008 & 10 \\
\hline 216 & SA17\#21 & 28May2006 & 07Jun2006 & 10 & 252 & SA19\#15 & 29Mar2008 & 08Apr2008 & 10 \\
\hline YEAR 17 & & & & & 253 & SA19\#16 & 08Apr2008 & 18Apr2008 & 10 \\
\hline 217 & SA18\#1 & 17Jun2006 & 27Jun2006 & 10 & 254 & SA19\#17 & 18Apr2008 & 28Apr2008 & 10 \\
\hline 218 & SA18\#2 & 27Jun2006 & 17Jul2006 & 20 & 255 & SA19\#18 & 28Apr2008 & 08May2008 & 10 \\
\hline 219 & SA18\#3 & 17Jul2006 & 03Aug2006 & 17 & 256 & SA19\#19 & 08May2008 & 18May2008 & 10 \\
\hline 220 & SA18\#4 & 03Aug2006 & 23Aug2006 & 20 & 257 & SA19\#20 & 18May2008 & 28May2008 & 10 \\
\hline 221 & SA18\#5 & 23Aug2006 & 02Sep2006 & 10 & 258 & SA19\#21 & 28May2008 & 07Jun2008 & 10 \\
\hline 222 & SA18\#6 & 02Sep2006 & 12Sep2006 & 10 & YEAR 19 & & & & \\
\hline 223 & SA18\#7 & 12Sep2006 & 22Sep2006 & 10 & 259 & SA20\#1 & 20Jun2008 & 27Jun2008 & 7 \\
\hline 224 & SA18\#8 & 22Sep2006 & $02 \mathrm{Oct} 2006$ & 10 & 260 & SA20\#2 & 27Jun2008 & 07Jul2008 & 10 \\
\hline 225 & SA18\#9 & $02 \mathrm{Oct} 2006$ & $22 \mathrm{Oct} 2006$ & 20 & 261 & SA20\#3 & 07Jul2008 & 17Jul2008 & 10 \\
\hline 226 & SA18\#10 & $22 \mathrm{Oct} 2006$ & 09Nov2006 & 18 & 262 & SA20\#4 & 17Jul2008 & 27Jul2008 & 10 \\
\hline 227 & SA18\#11 & 09Nov2006 & 27Nov2006 & 18 & 263 & SA20\#5 & 27Jul2008 & 03Aug2008 & 7 \\
\hline 228 & SA18\#12 & 27Nov2006 & 22Jan2007 & 56 & 264 & SA20\#6 & 03Aug2008 & 23Aug2008 & 20 \\
\hline 229 & SA18\#13 & 22Jan2007 & 28Feb2007 & 37 & 265 & SA20\#7 & 23Aug2008 & 02Sep2008 & 10 \\
\hline 230 & SA18\#14 & $28 \mathrm{Feb} 2007$ & 19Mar2007 & 19 & 266 & SA20\#8 & 02Sep2008 & 12Sep2008 & 10 \\
\hline 231 & SA18\#15 & 19Mar2007 & 08Apr2007 & 20 & 267 & SA20\#9 & 12Sep2008 & 22Sep2008 & 10 \\
\hline 232 & SA18\#16 & 08Apr2007 & 18Apr2007 & 10 & 268 & SA20\#10 & 22Sep2008 & 02Oct2008 & 10 \\
\hline 233 & SA18\#17 & 18Apr2007 & 28Apr2007 & 10 & 269 & SA20\#11 & $02 \mathrm{Oct} 2008$ & $22 \mathrm{Oct} 2008$ & 20 \\
\hline 234 & SA18\#18 & 28Apr2007 & 08May2007 & 10 & 270 & SA20\#12 & $22 \mathrm{Oct} 2008$ & 09Nov2008 & 18 \\
\hline 235 & SA18\#19 & 08Мay2007 & 18Мay2007 & 10 & 271 & SA20\#13 & 09Nov2008 & 27Nov2008 & 18 \\
\hline 236 & SA18\#20 & 18May2007 & 28May2007 & 10 & 272 & SA20\#14 & 27Nov2008 & 22Jan2009 & 56 \\
\hline 237 & SA18\#21 & 28May2007 & 07Jun2007 & 10 & 273 & SA20\#15 & 22Jan2009 & 28Feb2009 & 37 \\
\hline YEAR 18 & & & & & 274 & SA20\#16 & 28Feb2009 & 19Mar2009 & 19 \\
\hline 238 & SA19\#1 & 17Jul2007 & 27Jul2007 & 10 & 275 & SA20\#17 & 19Mar2009 & 08Apr2009 & 20 \\
\hline 239 & SA19\#2 & 27Jul2007 & 03Aug2007 & 7 & 276 & SA20\#18 & 08Apr2009 & 28Apr2009 & 20 \\
\hline 240 & SA19\#3 & 03Aug2007 & 23Aug2007 & 20 & 277 & SA20\#19 & 28Apr2009 & 08May2009 & 10 \\
\hline 241 & SA19\#4 & 23Aug2007 & 02Sep2007 & 10 & 278 & SA20\#20 & 08Мау2009 & 28Мay2009 & 20 \\
\hline 242 & SA19\#5 & 02Sep2007 & 12Sep2007 & 10 & 279 & SA20\#21 & 28May2009 & 07Jun2009 & 10 \\
\hline 243 & SA19\#6 & 12Sep2007 & 22Sep2007 & 10 & YEAR 20 & & & & \\
\hline 244 & SA19\#7 & 22Sep2007 & $02 \mathrm{Oct} 2007$ & 10 & 280 & SA21\#01 & 25Jun2009 & 05Jul2009 & 10 \\
\hline 245 & SA19\#8 & 02Oct2007 & $22 \mathrm{Oct} 2007$ & 20 & 281 & SA21\#02 & 05Jul2009 & 15Jul2009 & 10 \\
\hline 246 & SA19\#9 & $22 \mathrm{Oct} 2007$ & 09Nov2007 & 18 & 282 & SA21\#03 & 15Jul2009 & 25Jul2009 & 10 \\
\hline 247 & SA19\#10 & 09Nov2007 & 27Nov2007 & 18 & 283 & SA21\#04 & 25Jul2009 & 04Aug2009 & 10 \\
\hline 248 & SA19\#11 & 27 Nov2007 & 22Jan2008 & 56 & & & & & \\
\hline
\end{tabular}

Table 3. Sample dates and intervals of the sediment trap materials recovered from Stations AB, 1990-2009.

\begin{tabular}{|c|c|c|c|c|c|c|c|c|c|}
\hline Sequential \# & Sample \# & Open Date & Closed Date & Interval (d) & Sequential \# & Sample \# & Open Date & Closed Date & Interval (d) \\
\hline YEAR 1 & & & & & 15 & $\mathrm{AB} 2 \# 2$ & 23Aug1991 & 12Sep1991 & 20 \\
\hline 1 & $\mathrm{AB} 1 \# 1$ & 07Aug1990 & 23Aug 1990 & 16 & 16 & $\mathrm{AB} 2 \# 3$ & 12Sep1991 & $02 \mathrm{Oct} 1991$ & 20 \\
\hline 2 & $\mathrm{AB} 1 \# 2$ & 23Aug1990 & 12Sep1990 & 20 & 17 & $\mathrm{AB} 2 \# 4$ & 02 Oct1991 & 27Nov1991 & 56 \\
\hline 3 & $\mathrm{AB} 1 \# 3$ & 12Sep1990 & 02 Oct1990 & 20 & 18 & $\mathrm{AB} 2 \# 5$ & 27Nov1991 & 22Jan1992 & 56 \\
\hline 4 & $\mathrm{AB} 1 \# 4$ & 02Oct1990 & 27Nov1990 & 56 & 19 & $\mathrm{AB} 2 \# 6$ & 22Jan1992 & 19Mar1992 & 57 \\
\hline 5 & $\mathrm{AB} 1 \# 5$ & 27 Nov 1990 & 22Jan1991 & 56 & 20 & $\mathrm{AB} 2 \# 7$ & 19Mar1992 & 08Apr1992 & 20 \\
\hline 6 & $\mathrm{AB} 1 \# 6$ & 22Jan1991 & 19Mar1991 & 56 & 21 & AB2\#8 & 08Apr1992 & 28Apr1992 & 20 \\
\hline 7 & $\mathrm{AB} 1 \# 7$ & 19Mar1991 & 08Apr1991 & 20 & 22 & AB2\#9 & 28Apr1992 & 18May1992 & 20 \\
\hline 8 & $\mathrm{AB} 1 \# 8$ & 08Apr1991 & 28Apr1991 & 20 & 23 & $\mathrm{AB} 2 \# 10$ & 18May1992 & 07Jun1992 & 20 \\
\hline 9 & $\mathrm{AB} 1 \# 9$ & 28Apr1991 & 18May1991 & 20 & 24 & AB2\#11 & 07Jun1992 & 27Jun1992 & 20 \\
\hline 10 & AB1\#10 & 18Мay1991 & 07Jun1991 & 20 & 25 & AB2\#12 & 27Jun1992 & 17Jul1992 & 20 \\
\hline 11 & AB1\#11 & 07Jun1991 & 27Jun1991 & 20 & 26 & AB2\#13 & 17Jul1992 & 31Jul1992 & 14 \\
\hline 12 & $\mathrm{AB} 1 \# 12$ & 27Jun1991 & 17Jul1991 & 20 & YEAR 3 & & & & \\
\hline 13 & AB1\#13 & 17Jul1991 & 01Aug1991 & 15 & 27 & AB3\#1 & 07Aug1992 & 23Aug1992 & 16 \\
\hline YEAR 2 & & & & & 28 & AB3\#2 & 23Aug1992 & 12Sep1992 & 20 \\
\hline 14 & AB2\#1 & 07Aug1991 & 23Aug1991 & 16 & 29 & $\mathrm{AB} 3 \# 3$ & 12Sep1992 & 02Oct1992 & 20 \\
\hline
\end{tabular}


Kozo Takahashi, Hirofumi Asahi, Yusuke Okazaki, Jonaotaro Onodera, Hideto Tsutsui,

Takahito Ikenoue, Yoshiyuki Kanematsu, Seiji Tanaka and Shinya Iwasaki

Table 3 (Cont.)

\begin{tabular}{|c|c|c|c|c|c|c|c|c|c|}
\hline Sequential \# & Sample \# & Open Date & Closed Date & Interval (d) & Sequential \# & Sample \# & Open Date & Closed Date & Interval (d) \\
\hline 30 & $\mathrm{AB} 3 \# 4$ & 02Oct1992 & 22Oct1992 & 20 & 84 & $\mathrm{AB} 7 \# 6$ & 27Nov1996 & 22Jan1997 & 56 \\
\hline 31 & $\mathrm{AB} 3 \# 5$ & 22Oct1992 & 27Nov1992 & 36 & 85 & AB7\#7 & 22Jan1997 & 19Mar1997 & 56 \\
\hline 32 & AB3\#6 & 27Nov1992 & 22Jan1993 & 56 & 86 & $\mathrm{AB} 7 \# 8$ & 19Mar1997 & 08Apr1997 & 20 \\
\hline 33 & AB3\#7 & 22Jan1993 & 19Mar1993 & 56 & 87 & $\mathrm{AB} 7 \# 9$ & 08Apr1997 & 28Apr1997 & 20 \\
\hline 34 & AB3\#8 & 19Mar1993 & 08Apr1993 & 20 & 88 & AB7\#10 & 28Apr1997 & 18May1997 & 20 \\
\hline 35 & AB3\#9 & 08Apr1993 & 28Apr1993 & 20 & 89 & AB7\#11 & 18May1997 & 07Jun1997 & 20 \\
\hline 36 & AB3\#10 & 28Apr1993 & 18May1993 & 20 & 90 & $\mathrm{AB} 7 \# 12$ & 07Jun1997 & 27Jun1997 & 20 \\
\hline 37 & AB3\#11 & 18May1993 & 07Jun1993 & 20 & 91 & $\mathrm{AB} 7 \# 13$ & 27Jun1997 & 01Aug1997 & 35 \\
\hline 38 & AB3\#12 & 07Jun1993 & 27Jun1993 & 20 & YEAR 8 & & & & \\
\hline 39 & AB3\#13 & 27Jun1993 & 20Jul1993 & 23 & 92 & AB8\#1 & 08Aug1997 & 23Aug1997 & 15 \\
\hline YEAR 4 & & & & & 93 & AB8\#2 & 23Aug1997 & 12Sep1997 & 20 \\
\hline 40 & AB4\#1 & 02Aug1993 & 23Aug1993 & 21 & 94 & $\mathrm{AB} 8 \# 3$ & 12Sep1997 & $02 \mathrm{Oct} 1997$ & 20 \\
\hline 41 & $\mathrm{AB} 4 \# 2$ & 23Aug1993 & 12Sep1993 & 20 & 95 & AB8\#4 & 02 Oct1997 & 22Oct1997 & 20 \\
\hline 42 & AB4\#3 & 12Sep1993 & 02 Oct1993 & 20 & 96 & AB8\#5 & 22Oct1997 & 27Nov1997 & 36 \\
\hline 43 & AB4\#4 & 02 Oct1993 & 22 Oct1993 & 20 & 97 & AB8\#6 & 27Nov1997 & 22Jan1998 & 56 \\
\hline 44 & $\mathrm{AB} 4 \# 5$ & 22 Oct1993 & 27Nov1993 & 36 & 98 & AB8\#7 & 22Jan1998 & 19Mar1998 & 56 \\
\hline 45 & AB4\#6 & 27Nov1993 & 22Jan1994 & 56 & 99 & $\mathrm{AB} 8 \# 8$ & 19Mar1998 & 08Apr1998 & 20 \\
\hline 46 & AB4\#7 & 22Jan1994 & 19Mar1994 & 56 & 100 & AB8\#9 & 08Apr1998 & 28Apr1998 & 20 \\
\hline 47 & $\mathrm{AB} 4 \# 8$ & 19Mar1994 & 08Apr1994 & 20 & 101 & AB8\#10 & 28Apr1998 & 18May1998 & 20 \\
\hline 48 & AB4\#9 & 08Apr1994 & 28Apr1994 & 20 & 102 & AB8\#11 & 18May1998 & 07Jun1998 & 20 \\
\hline 49 & AB4\#10 & 28Apr1994 & 18May1994 & 20 & 103 & AB8\#12 & 07Jun1998 & 27Jun1998 & 20 \\
\hline 50 & AB4\#11 & 18May1994 & 07Jun1994 & 20 & 104 & AB8\#13 & 27Jun1998 & 01Aug1998 & 35 \\
\hline 51 & AB4\#12 & 07Jun1994 & 27Jun1994 & 20 & YEAR 9 & & & & \\
\hline 52 & AB4\#13 & 27Jun1994 & 25Jul1994 & 28 & 105 & AB9\#1 & 07Aug1998 & 23Aug1998 & 16 \\
\hline YEAR 5 & & & & & 106 & AB9\#2 & 23Aug1998 & 12Sep1998 & 20 \\
\hline 53 & AB5\#1 & 04Aug1994 & 23Aug1994 & 19 & 107 & AB9\#3 & 12Sep1998 & 02Oct1998 & 20 \\
\hline 54 & AB5\#2 & 23Aug1994 & 12Sep1994 & 20 & 108 & AB9\#4 & 02 Oct1998 & 22Oct1998 & 20 \\
\hline 55 & AB5\#3 & 12Sep1994 & $02 \mathrm{Oct} 1994$ & 20 & 109 & AB9\#5 & 22Oct1998 & 27Nov1998 & 36 \\
\hline 56 & AB5\#4 & 02Oct1994 & 22Oct1994 & 20 & 110 & AB9\#6 & 27Nov1998 & 22Jan1999 & 56 \\
\hline 57 & $\mathrm{AB} 5 \# 5$ & 22 Oct1994 & 27Nov1994 & 36 & 111 & AB9\#7 & 22Jan1999 & 19Mar1999 & 56 \\
\hline 58 & AB5\#6 & 27Nov1994 & 22Jan1995 & 56 & 112 & AB9\#8 & 19Mar1999 & 08Apr1999 & 20 \\
\hline 59 & AB5\#7 & 22Jan1995 & 19Mar1995 & 56 & 113 & AB9\#9 & 08Apr1999 & 28Apr1999 & 20 \\
\hline 60 & AB5\#8 & 19Mar1995 & 08Apr1995 & 20 & 114 & AB9\#10 & 28Apr1999 & 18May1999 & 20 \\
\hline 61 & AB5\#9 & 08Apr1995 & 28Apr1995 & 20 & 115 & AB9\#11 & 18May1999 & 07Jun1999 & 20 \\
\hline 62 & AB5\#10 & 28Apr1995 & 13May1995 & 15 & 116 & AB9\#12 & 07Jun1999 & 27Jun1999 & 20 \\
\hline 63 & AB5\#11 & 13Маy1995 & 28May1995 & 15 & 117 & AB9\#13 & 27Jun1999 & 03Aug1999 & 37 \\
\hline 64 & AB5\#12 & 28May1995 & 17Jun1995 & 20 & YEAR 13* & & & & \\
\hline 65 & AB5\#13 & 17Jun1995 & 25Jul1995 & 38 & 118 & AB14\#1 & 15Aug2002 & 23Aug2002 & 8 \\
\hline YEAR 6 & & & & & 119 & $\mathrm{AB} 14 \# 2$ & 23Aug2002 & 02Sep2002 & 10 \\
\hline 66 & AB6\#1 & 07Aug1995 & 23Aug1995 & 16 & 120 & AB14\#3 & 02Sep2002 & 12Sep2002 & 10 \\
\hline 67 & AB6\#2 & 23Aug1995 & 12Sep1995 & 20 & 121 & $\mathrm{AB} 14 \# 4$ & 12Sep2002 & 22Sep2002 & 10 \\
\hline 68 & $\mathrm{AB} 6 \# 3$ & 12Sep1995 & $02 \mathrm{Oct} 1995$ & 20 & 122 & AB14\#5 & 22Sep2002 & $02 \mathrm{Oct} 2002$ & 10 \\
\hline 69 & AB6\#4 & 02Oct1995 & 22Oct1995 & 20 & 123 & AB14\#6 & $02 \mathrm{Oct} 2002$ & $12 \mathrm{Oct} 2002$ & 10 \\
\hline 70 & $\mathrm{AB} 6 \# 5$ & 22 Oct1995 & 27Nov1995 & 36 & 124 & AB14\#7 & $12 \mathrm{Oct} 2002$ & $22 \mathrm{Oct} 2002$ & 10 \\
\hline 71 & AB6\#6 & 27 Nov1995 & 22Jan1996 & 56 & 125 & AB14\#8 & $22 \mathrm{Oct} 2002$ & 27Nov2002 & 36 \\
\hline 72 & $\mathrm{AB} 6 \# 7$ & 22Jan1996 & 19Mar1996 & 57 & 126 & $\mathrm{AB} 14 \# 9$ & 27 Nov2002 & 22Jan2003 & 56 \\
\hline 73 & $\mathrm{AB} 6 \# 8$ & 19Mar1996 & 08Apr1996 & 20 & 127 & $\mathrm{AB} 14 \# 10$ & 22Jan2003 & 19Mar2003 & 56 \\
\hline 74 & AB6\#9 & 08Apr1996 & 28Apr1996 & 20 & 128 & AB14\#11 & 19Mar2003 & 29Mar2003 & 10 \\
\hline 75 & AB6\#10 & 28Apr1996 & 18May1996 & 20 & 129 & $\mathrm{AB} 14 \# 12$ & 29Mar2003 & 08Apr2003 & 10 \\
\hline 76 & AB6\#11 & 18May1996 & 07Jun1996 & 20 & 130 & AB14\#13 & 08Apr2003 & 18Apr2003 & 10 \\
\hline 77 & AB6\#12 & 07Jun1996 & 27Jun1996 & 20 & 131 & AB14\#14 & 18Apr2003 & 28Apr2003 & 10 \\
\hline 78 & AB6\#13 & 27Jun1996 & 25Jul1996 & 28 & 132 & AB14\#15 & 28Apr2003 & 08May2003 & 10 \\
\hline YEAR 7 & & & & & 133 & AB14\#16 & 08May2003 & 18May2003 & 10 \\
\hline 79 & $\mathrm{AB} 7 \# 1$ & 08Aug1996 & 23Aug1996 & 15 & 134 & AB14\#17 & 18May2003 & 28May2003 & 10 \\
\hline 80 & $\mathrm{AB} 7 \# 2$ & 23Aug1996 & 12Sep1996 & 20 & 135 & AB14\#18 & 28May2003 & 07Jun2003 & 10 \\
\hline 81 & $\mathrm{AB} 7 \# 3$ & 12Sep1996 & $02 \mathrm{Oct} 1996$ & 20 & 136 & AB14\#19 & 07Jun2003 & 17Jun2003 & 10 \\
\hline 82 & $\mathrm{AB} 7 \# 4$ & 02Oct1996 & 22 Oct1996 & 20 & 137 & $\mathrm{AB} 14 \# 20$ & 17Jun2003 & 27Jun2003 & 10 \\
\hline 83 & $\mathrm{AB} 7 \# 5$ & $22 \mathrm{Oct} 1996$ & 27Nov1996 & 36 & 138 & AB14\#21 & 27Jun2003 & 10Jul2003 & 13 \\
\hline
\end{tabular}


Table 3 (Cont.)

\begin{tabular}{|c|c|c|c|c|c|c|c|c|c|}
\hline Sequential \# & Sample \# & Open Date & Closed Date & Interval (d) & Sequential \# & Sample \# & Open Date & Closed Date & Interval (d) \\
\hline YEAR 14 & & & & & 186 & AB18\#17 & 18Apr2007 & 28Apr2007 & 10 \\
\hline 139 & AB15\#1 & 13Jul2003 & 03Aug2003 & 21 & 187 & AB18\#18 & 28Apr2007 & 08Мay2007 & 10 \\
\hline 140 & AB15\#2 & 03Aug2003 & 23Aug2003 & 20 & 188 & AB18\#19 & 08Мау2007 & 18Мay2007 & 10 \\
\hline 141 & AB15\#3 & 23Aug2003 & 02Sep2003 & 10 & 189 & AB18\#20 & 18May2007 & 28May2007 & 10 \\
\hline 142 & AB15\#4 & 02Sep2003 & 12Sep2003 & 10 & 190 & AB18\#21 & 28Мay2007 & 07Jun2007 & 10 \\
\hline 143 & AB15\#5 & 12Sep2003 & 22Sep2003 & 10 & YEAR 18 & & & & \\
\hline 144 & AB15\#6 & 22Sep2003 & $02 \mathrm{Oct} 2003$ & 10 & 191 & AB19\#1 & 17Jul2007 & 27Jul2007 & 10 \\
\hline 145 & $\mathrm{AB} 15 \# 7$ & $02 \mathrm{Oct} 2003$ & $12 \mathrm{Oct} 2003$ & 10 & 192 & AB19\#2 & 27Jul2007 & 03Aug2007 & 7 \\
\hline 146 & $\mathrm{AB} 15 \# 8$ & $12 \mathrm{Oct} 2003$ & $22 \mathrm{Oct} 2003$ & 10 & 193 & AB19\#3 & 03Aug2007 & 23Aug2007 & 20 \\
\hline 147 & AB15\#9 & $22 \mathrm{Oct} 2003$ & 27 Nov2003 & 36 & 194 & AB19\#4 & 23Aug2007 & 02Sep2007 & 10 \\
\hline 148 & AB15\#10 & 27 Nov2003 & 22Jan2004 & 56 & 195 & AB19\#5 & 02Sep2007 & 12Sep2007 & 10 \\
\hline YEAR 15 & & & & & 196 & AB19\#6 & 12Sep2007 & 22Sep2007 & 10 \\
\hline 149 & AB16\#1 & 14Aug2004 & 23Aug2004 & 9 & 197 & AB19\#7 & 22Sep2007 & $02 \mathrm{Oct} 2007$ & 10 \\
\hline 150 & AB16\#2 & 23Aug2004 & 02Sep2004 & 10 & 198 & AB19\#8 & $02 \mathrm{Oct} 2007$ & $22 \mathrm{Oct} 2007$ & 20 \\
\hline 151 & AB16\#3 & 02Sep2004 & 12Sep2004 & 10 & 199 & AB19\#9 & $22 \mathrm{Oct} 2007$ & 09Nov2007 & 18 \\
\hline 152 & AB16\#4 & 12Sep2004 & 22Sep2004 & 10 & 200 & AB19\#10 & 09Nov2007 & 27 Nov2007 & 18 \\
\hline 153 & AB16\#5 & 22Sep2004 & $02 \mathrm{Oct} 2004$ & 10 & 201 & AB19\#11 & 27 Nov2007 & 22Jan2008 & 56 \\
\hline 154 & AB16\#6 & $02 \mathrm{Oct} 2004$ & $12 \mathrm{Oct} 2004$ & 10 & 202 & AB19\#12 & 22Jan2008 & 28Feb2008 & 37 \\
\hline 155 & AB16\#7 & $12 \mathrm{Oct} 2004$ & $22 \mathrm{Oct} 2004$ & 10 & 203 & AB19\#13 & $28 \mathrm{Feb} 2008$ & 19Mar2008 & 20 \\
\hline 156 & AB16\#8 & $22 \mathrm{Oct} 2004$ & 27 Nov2004 & 36 & 204 & AB19\#14 & 19Mar2008 & 29Mar2008 & 10 \\
\hline 157 & AB16\#9 & 27 Nov2004 & 22Jan2005 & 56 & 205 & AB19\#15 & 29Mar2008 & 08Apr2008 & 10 \\
\hline 158 & AB16\#10 & 22Jan2005 & 19Mar2005 & 56 & 206 & AB19\#16 & 08Apr2008 & 18Apr2008 & 10 \\
\hline 159 & AB16\#11 & 19Mar2005 & 29Mar2005 & 10 & 207 & AB19\#17 & 18Apr2008 & 28Apr2008 & 10 \\
\hline 160 & AB16\#12 & 29Mar2005 & 08Apr2005 & 10 & 208 & AB19\#18 & 28Apr2008 & 08May2008 & 10 \\
\hline 161 & AB16\#13 & 08Apr2005 & 18Apr2005 & 10 & 209 & AB19\#19 & 08May2008 & 18May2008 & 10 \\
\hline 162 & AB16\#14 & 18Apr2005 & 28Apr2005 & 10 & 210 & AB19\#20 & 18May2008 & 28May2008 & 10 \\
\hline 163 & AB16\#15 & 28Apr2005 & 08Мay2005 & 10 & 211 & AB19\#21 & 28Мay2008 & 07Jun2008 & 10 \\
\hline 164 & AB16\#16 & 08May2005 & 18May2005 & 10 & YEAR 19 & & & & \\
\hline 165 & AB16\#17 & 18May2005 & 28May2005 & 10 & 212 & AB20\#1 & 20Jun2008 & 27Jun2008 & 7 \\
\hline 166 & AB16\#18 & 28May2005 & 07Jun2005 & 10 & 213 & AB20\#2 & 27Jun2008 & 07Jul2008 & 10 \\
\hline 167 & AB16\#19 & 07Jun2005 & 17Jun2005 & 10 & 214 & AB20\#3 & 07Jul2008 & 17Jul2008 & 10 \\
\hline 168 & $\mathrm{AB} 16 \# 20$ & 17Jun2005 & 27Jun2005 & 10 & 215 & AB20\#4 & 17Jul2008 & 27Jul2008 & 10 \\
\hline 169 & $\mathrm{AB} 16 \# 21$ & 27Jun2005 & 06Jul2005 & 9 & 216 & AB20\#5 & 27Jul2008 & 03Aug2008 & 7 \\
\hline YEAR $17 *$ & & & & & 217 & AB20\#6 & 03Aug2008 & 23Aug2008 & 20 \\
\hline 170 & AB18\#1 & 17Jun2006 & 27Jun2006 & 10 & 218 & $\mathrm{AB} 20 \# 7$ & 23Aug2008 & 02Sep2008 & 10 \\
\hline 171 & $\mathrm{AB} 18 \# 2$ & 27Jun2006 & 17Jul2006 & 20 & 219 & AB20\#8 & 02Sep2008 & 12Sep2008 & 10 \\
\hline 172 & AB18\#3 & 17Jul2006 & 03Aug2006 & 17 & 220 & AB20\#9 & 12Sep2008 & 22Sep2008 & 10 \\
\hline 173 & AB18\#4 & 03Aug2006 & 23Aug2006 & 20 & 221 & AB20\#10 & 22Sep2008 & $02 \mathrm{Oct} 2008$ & 10 \\
\hline 174 & AB18\#5 & 23Aug2006 & 02Sep2006 & 10 & 222 & AB20\#11 & $02 \mathrm{Oct} 2008$ & $22 \mathrm{Oct} 2008$ & 20 \\
\hline 175 & AB18\#6 & 02Sep2006 & 12Sep2006 & 10 & 223 & AB20\#12 & $22 \mathrm{Oct} 2008$ & 09Nov2008 & 18 \\
\hline 176 & AB18\#7 & 12Sep2006 & 22Sep2006 & 10 & 224 & AB20\#13 & 09Nov2008 & 27 Nov2008 & 18 \\
\hline 177 & AB18\#8 & 22Sep2006 & $02 \mathrm{Oct} 2006$ & 10 & 225 & AB20\#14 & 27 Nov2008 & 22Jan2009 & 56 \\
\hline 178 & $\mathrm{AB} 18 \# 9$ & $02 \mathrm{Oct} 2006$ & $22 \mathrm{Oct} 2006$ & 20 & 226 & AB20\#15 & 22Jan2009 & 28Feb2009 & 37 \\
\hline 179 & AB18\#10 & $22 \mathrm{Oct} 2006$ & 09Nov2006 & 18 & 227 & AB20\#16 & 28Feb2009 & 19Mar2009 & 19 \\
\hline 180 & AB18\#11 & 09Nov2006 & 27Nov2006 & 18 & 228 & AB20\#17 & 19Mar2009 & 08Apr2009 & 20 \\
\hline 181 & AB18\#12 & 27 Nov2006 & 22Jan2007 & 56 & 229 & AB20\#18 & 08Apr2009 & 28Apr2009 & 20 \\
\hline 182 & AB18\#13 & 22Jan2007 & $28 \mathrm{Feb} 2007$ & 37 & 230 & AB20\#19 & 28Apr2009 & 08Мay2009 & 10 \\
\hline 183 & AB18\#14 & 28Feb2007 & 19Mar2007 & 19 & 231 & AB20\#20 & 08May2009 & 28May2009 & 20 \\
\hline 184 & AB18\#15 & 19Mar2007 & 08Apr2007 & 20 & 232 & AB20\#21 & 28May2009 & 07Jun2009 & 10 \\
\hline 185 & AB18\#16 & 08Apr2007 & 18Apr2007 & 10 & & & & & \\
\hline
\end{tabular}

No sample is available for YEARs 10-12, 16, and 20 at Station AB due to hiatuses in trap deployment or unsuccessful trap mooring recovery. Starting from the trap YEAR 13 in 2002 at Station AB the sample identification was designated as AB14 in order to synchronize with that of Station SA, by skipping AB13 (Table 4). 
Kozo Takahashi, Hirofumi Asahi, Yusuke Okazaki, Jonaotaro Onodera, Hideto Tsutsui,

Takahito Ikenoue, Yoshiyuki Kanematsu, Seiji Tanaka and Shinya Iwasaki

Table 4. List of archival filter samples from Station SA.

\begin{tabular}{|c|c|c|c|c|c|c|c|c|}
\hline Sample ID & Size $(\mu \mathrm{m})$ & $\begin{array}{l}\text { Aliquot } \\
\text { size \& ID }\end{array}$ & Sample ID & Size $(\mu \mathrm{m})$ & $\begin{array}{l}\text { Aliquot } \\
\text { size \& ID }\end{array}$ & Sample ID & Size $(\mu \mathrm{m})$ & $\begin{array}{l}\text { Aliquot } \\
\text { size \& ID }\end{array}$ \\
\hline SA2\#1 & $63-1000$ & $1 / 256$ & $\overline{\mathrm{SA} 2 \# 7}$ & $<1000$ & $1 / 16384 \mathrm{C}$ & SA3\#3 & $<63$ & $1 / 1024 \mathrm{~A}$ \\
\hline SA2\#1 & $<1000$ & $1 / 1024 \mathrm{C}$ & $\mathrm{SA} 2 \# 7$ & $<1000$ & $1 / 16384 \mathrm{D}$ & SA3\#3 & $<63$ & $1 / 1024 \mathrm{C}$ \\
\hline SA2\#1 & $<1000$ & 1/4096B & SA2\#7 & $<63$ & $1 / 1024 \mathrm{~A}$ & SA3\#4 & 63-1000 & $1 / 256 \mathrm{~B}$ \\
\hline $\mathrm{SA} 2 \# 1$ & $<1000$ & $1 / 16384 \mathrm{~A}$ & $\mathrm{SA} 2 \# 7$ & $<63$ & 1/1024B & SA3\#4 & $63-1000$ & 1/1024B \\
\hline SA2\#1 & $<1000$ & 1/16384B & SA2\#7 & $<63$ & 1/1024D & SA3\#4 & $63-1000$ & $1 / 1024 \mathrm{C}$ \\
\hline SA2\#1 & $<1000$ & $1 / 16384 \mathrm{C}$ & $\mathrm{SA} 2 \# 8$ & $63-1000$ & $1 / 256 \mathrm{~A}$ & SA3\#4 & $<1000$ & 1/1024B \\
\hline SA2\#1 & $<1000$ & $1 / 16384 \mathrm{D}$ & SA2\#8 & $<1000$ & 1/1024B & SA3\#4 & $<1000$ & $1 / 1024 \mathrm{C}$ \\
\hline $\mathrm{SA} 2 \# 2$ & $63-1000$ & $1 / 256 \mathrm{~A}$ & $\mathrm{SA} 2 \# 8$ & $<1000$ & $1 / 1024 \mathrm{C}$ & SA3\#4 & $<1000$ & $1 / 4096 \mathrm{C}$ \\
\hline $\mathrm{SA} 2 \# 2$ & $63-1000$ & $1 / 256 \mathrm{~B}$ & SA2\#8 & $<1000$ & 1/16384B & SA3\#4 & $<63$ & $1 / 64$ \\
\hline $\mathrm{SA} 2 \# 2$ & $63-1000$ & $1 / 1024$ & $\mathrm{SA} 2 \# 8$ & $<1000$ & $1 / 16384 \mathrm{C}$ & $\mathrm{SA} 3 \# 4$ & $<63$ & $1 / 256 \mathrm{~A}$ \\
\hline $\mathrm{SA} 2 \# 2$ & $<1000$ & 1/1024B & SA2\#8 & $<1000$ & $1 / 16384 \mathrm{D}$ & $\mathrm{SA} 3 \# 4$ & $<63$ & $1 / 256 \mathrm{~B}$ \\
\hline $\mathrm{SA} 2 \# 2$ & $<1000$ & $1 / 1024 \mathrm{C}$ & SA2\#8 & $<63$ & $1 / 64(1)$ & SA3\#4 & $<63$ & 1/1024B \\
\hline $\mathrm{SA} 2 \# 2$ & $<1000$ & 1/4096B & SA2\#8 & $<63$ & $1 / 64(2)$ & $\mathrm{SA} 3 \# 4$ & $<63$ & $1 / 1024 \mathrm{C}$ \\
\hline $\mathrm{SA} 2 \# 2$ & $<1000$ & $1 / 16384 \mathrm{D}$ & SA2\#8 & $<63$ & $1 / 1024 \mathrm{~A}$ & $\mathrm{SA} 3 \# 5$ & $63-1000$ & $1 / 256 \mathrm{~B}$ \\
\hline SA2\#2 & $<63$ & $1 / 64$ & SA2\#8 & $<63$ & 1/1024B & SA3\#5 & $63-1000$ & 1/1024B \\
\hline SA2\#3 & $63-1000$ & $1 / 256 \mathrm{~A}$ & SA2\#8 & $<63$ & $1 / 1024 \mathrm{D}$ & SA3\#5 & $63-1000$ & $1 / 1024 \mathrm{C}$ \\
\hline $\mathrm{SA} 2 \# 3$ & $<1000$ & 1/1024B & $\overline{\text { SA3\#1 }}$ & $63-1000$ & $1 / 256 \mathrm{~B}$ & SA3\#5 & $<1000$ & 1/1024B \\
\hline $\mathrm{SA} 2 \# 3$ & $<1000$ & $1 / 1024 \mathrm{C}$ & SA3\#1 & $63-1000$ & $1 / 1024 \mathrm{C}$ & SA3\#5 & $<1000$ & $1 / 1024 \mathrm{C}$ \\
\hline $\mathrm{SA} 2 \# 3$ & $<1000$ & 1/16384D & SA3\#1 & $<1000$ & 1/1024B & SA3\#5 & $<1000$ & $1 / 4096 \mathrm{C}$ \\
\hline $\mathrm{SA} 2 \# 4$ & $63-1000$ & $1 / 256 \mathrm{~A}$ & SA3\#1 & $<1000$ & $1 / 1024 \mathrm{C}$ & SA3\#5 & $<1000$ & 1/16384D \\
\hline $\mathrm{SA} 2 \# 4$ & $<1000$ & $1 / 1024 \mathrm{~B}$ & SA3\#1 & $<1000$ & $1 / 16384 \mathrm{C}$ & SA3\#5 & $<63$ & $1 / 64$ \\
\hline $\mathrm{SA} 2 \# 4$ & $<1000$ & $1 / 1024 \mathrm{C}$ & SA3\#1 & $<1000$ & $1 / 16384 \mathrm{D}$ & SA3\#5 & $<63$ & $1 / 256 \mathrm{~A}$ \\
\hline $\mathrm{SA} 2 \# 4$ & $<1000$ & $1 / 4096 \mathrm{~B}$ & SA3\#1 & $<63$ & $1 / 256$ & SA3\#5 & $<63$ & $1 / 256 \mathrm{~B}$ \\
\hline $\mathrm{SA} 2 \# 4$ & $<1000$ & $1 / 16384 \mathrm{C}$ & SA3\#1 & $<63$ & $1 / 256$ & SA3\#5 & $<63$ & $1 / 1024 \mathrm{~A}$ \\
\hline $\mathrm{SA} 2 \# 4$ & $<1000$ & $1 / 16384 \mathrm{D}$ & SA3\#1 & $<63$ & $1 / 256$ & SA3\#5 & $<63$ & $1 / 1024 \mathrm{C}$ \\
\hline $\mathrm{SA} 2 \# 4$ & $<63$ & $1 / 64$ & SA3\#1 & $<63$ & $1 / 256 \mathrm{~A}$ & SA3\#6 & $63-1000$ & 1/1024B \\
\hline $\mathrm{SA} 2 \# 5$ & $63-1000$ & $1 / 256 \mathrm{~A}$ & SA3\#1 & $<63$ & $1 / 256 \mathrm{~B}$ & SA3\#6 & $63-1000$ & $1 / 1024 \mathrm{C}$ \\
\hline $\mathrm{SA} 2 \# 5$ & $63-1000$ & $1 / 256 \mathrm{~B}$ & SA3\#1 & $<63$ & $1 / 1024$ & SA3\#6 & $63-1000$ & \\
\hline $\mathrm{SA} 2 \# 5$ & $63-1000$ & $1 / 1024 \mathrm{~A}$ & SA3\#1 & $<63$ & $1 / 1024$ & SA3\#6 & $<1000$ & 1/1024B \\
\hline $\mathrm{SA} 2 \# 5$ & $63-1000$ & $1 / 1024 \mathrm{~B}$ & SA3\#1 & $<63$ & $1 / 1024$ & SA3\#6 & $<1000$ & $1 / 1024 \mathrm{C}$ \\
\hline $\mathrm{SA} 2 \# 5$ & $<1000$ & 1/1024B & SA3\#1 & $<63$ & $1 / 1024 \mathrm{~A}$ & SA3\#6 & $<1000$ & $1 / 4096 \mathrm{C}$ \\
\hline $\mathrm{SA} 2 \# 5$ & $<1000$ & $1 / 1024 \mathrm{C}$ & SA3\#2 & $63-1000$ & $1 / 256 \mathrm{~B}$ & SA3\#6 & $<63$ & $1 / 256 \mathrm{~A}$ \\
\hline $\mathrm{SA} 2 \# 5$ & $<1000$ & $1 / 4096 \mathrm{C}$ & SA3\#2 & $63-1000$ & $1 / 1024 \mathrm{~A}$ & SA3\#6 & $<63$ & $1 / 256 \mathrm{~B}$ \\
\hline $\mathrm{SA} 2 \# 5$ & $<1000$ & $1 / 16384 \mathrm{~A}$ & SA3\#2 & $63-1000$ & 1/1024B & SA3\#6 & $<63$ & $1 / 1024 \mathrm{~A}$ \\
\hline $\mathrm{SA} 2 \# 5$ & $<1000$ & 1/16384B & SA3\#2 & $<1000$ & 1/1024B & SA3\#6 & $<63$ & 1/1024B \\
\hline $\mathrm{SA} 2 \# 5$ & $<1000$ & $1 / 16384 \mathrm{C}$ & SA3\#2 & $<1000$ & $1 / 1024 \mathrm{C}$ & SA3\#7 & $63-1000$ & 1/1024B \\
\hline $\mathrm{SA} 2 \# 5$ & $<1000$ & $1 / 16384 \mathrm{D}$ & SA3\#2 & $<1000$ & 1/16384B & SA3\#7 & $63-1000$ & $1 / 1024 \mathrm{C}$ \\
\hline $\mathrm{SA} 2 \# 5$ & $<63$ & $1 / 64$ & SA3\#2 & $<1000$ & $1 / 16384 \mathrm{C}$ & SA3\#7 & $<1000$ & $1 / 256 \mathrm{~A}$ \\
\hline $\mathrm{SA} 2 \# 5$ & $<63$ & $1 / 256 \mathrm{~A}$ & $\mathrm{SA} 3 \# 2$ & $<1000$ & $1 / 16384 \mathrm{D}$ & SA3\#7 & $<1000$ & $1 / 256 \mathrm{~B}$ \\
\hline SA2\#6 & $63-1000$ & $1 / 256 \mathrm{~B}$ & SA3\#2 & $<63$ & $1 / 64$ & SA3\#7 & $<1000$ & $1 / 256 \mathrm{C}$ \\
\hline SA2\#6 & $<1000$ & $1 / 1024 \mathrm{~B}$ & SA3\#2 & $<63$ & $1 / 256 \mathrm{~A}$ & $\mathrm{SA} 3 \# 7$ & $<1000$ & 1/1024B \\
\hline SA2\#6 & $<1000$ & $1 / 1024 \mathrm{C}$ & SA3\#2 & $<63$ & $1 / 256 \mathrm{~B}$ & SA3\#7 & $<1000$ & $1 / 1024 \mathrm{C}$ \\
\hline SA2\#6 & $<1000$ & 1/4096B & SA3\#2 & $<63$ & $1 / 1024 \mathrm{~B}$ & $\mathrm{SA} 3 \# 7$ & $<1000$ & 1/16384B \\
\hline SA2\#6 & $<1000$ & $1 / 16384 \mathrm{~A}$ & SA3\#2 & $<63$ & $1 / 1024 \mathrm{C}$ & SA3\#7 & $<1000$ & $1 / 16384 \mathrm{C}$ \\
\hline SA2\#6 & $<1000$ & 1/16384B & SA3\#3 & $63-1000$ & $1 / 256 \mathrm{~B}$ & SA3\#7 & $<1000$ & 1/16384D \\
\hline $\mathrm{SA} 2 \# 6$ & $<1000$ & $1 / 16384 \mathrm{C}$ & SA3\#3 & $63-1000$ & $1 / 1024 \mathrm{~A}$ & SA3\#7 & $<63$ & $1 / 256 \mathrm{~A}$ \\
\hline SA2\#6 & $<1000$ & $1 / 16384 \mathrm{D}$ & SA3\#3 & $63-1000$ & 1/1024B & SA3\#7 & $<63$ & $1 / 256 \mathrm{~B}$ \\
\hline SA2\#6 & $<63$ & $1 / 64$ & SA3\#3 & $<1000$ & 1/1024B & SA3\#7 & $<63$ & $1 / 256 \mathrm{C}$ \\
\hline SA2\#6 & $<63$ & $1 / 1024 \mathrm{~A}$ & SA3\#3 & $<1000$ & $1 / 1024 \mathrm{C}$ & SA3\#7 & $<63$ & $1 / 1024 \mathrm{~A}$ \\
\hline $\mathrm{SA} 2 \# 6$ & $<63$ & $1 / 1024 \mathrm{~B}$ & SA3\#3 & $<1000$ & $1 / 4096 \mathrm{C}$ & SA3\#7 & $<63$ & $1 / 1024 \mathrm{C}$ \\
\hline $\mathrm{SA} 2 \# 6$ & $<63$ & $1 / 1024 \mathrm{D}$ & SA3\#3 & $<1000$ & 1/16384B & SA3\#8 & $63-1000$ & $1 / 256 \mathrm{~B}$ \\
\hline SA2\#7 & $63-1000$ & $1 / 256 \mathrm{~A}$ & SA3\#3 & $<1000$ & $1 / 16384 C$ & SA3\#8 & $63-1000$ & $1 / 1024 \mathrm{~A}$ \\
\hline $\mathrm{SA} 2 \# 7$ & $<1000$ & $1 / 1024 \mathrm{~B}$ & SA3\#3 & $<1000$ & 1/16384D & SA3\#8 & $63-1000$ & $1 / 1024 \mathrm{C}$ \\
\hline $\mathrm{SA} 2 \# 7$ & $<1000$ & $1 / 1024 \mathrm{C}$ & SA3\#3 & $<63$ & $1 / 64$ & SA3\#8 & $<1000$ & $1 / 256 \mathrm{~A}$ \\
\hline $\mathrm{SA} 2 \# 7$ & $<1000$ & 1/4096B & SA3\#3 & $<63$ & $1 / 256 \mathrm{~A}$ & $\mathrm{SA} 3 \# 8$ & $<1000$ & $1 / 256 \mathrm{~B}$ \\
\hline $\mathrm{SA} 2 \# 7$ & $<1000$ & 1/16384B & $\mathrm{SA} 3 \# 3$ & $<63$ & $1 / 256 \mathrm{~B}$ & SA3\#8 & $<1000$ & $1 / 256 \mathrm{C}$ \\
\hline
\end{tabular}


Table 4 (Cont.)

\begin{tabular}{|c|c|c|c|c|c|c|c|c|}
\hline Sample ID & Size $(\mu \mathrm{m})$ & $\begin{array}{l}\text { Aliquot } \\
\text { size \& ID }\end{array}$ & Sample ID & Size $(\mu \mathrm{m})$ & $\begin{array}{l}\text { Aliquot } \\
\text { size \& ID }\end{array}$ & Sample ID & Size $(\mu \mathrm{m})$ & $\begin{array}{l}\text { Aliquot } \\
\text { size \& ID }\end{array}$ \\
\hline SA3\#8 & $<1000$ & $1 / 256 \mathrm{C}$ & $\overline{\text { SA4\#3 }}$ & $<1000$ & $1 / 16384 \mathrm{D}$ & SA4\#7 & $<63$ & $1 / 1024 \mathrm{~B}$ \\
\hline SA3\#8 & $<1000$ & $1 / 1024 \mathrm{~B}$ & SA4\#3 & $<63$ & $1 / 256 \mathrm{~B}$ & SA4\#7 & $<63$ & $1 / 4096 \mathrm{~B}$ \\
\hline SA3\#8 & $<1000$ & $1 / 1024 \mathrm{C}$ & SA4\#3 & $<63$ & $1 / 1024 \mathrm{~A}$ & SA4\#8 & $63-1000$ & $1 / 1024 \mathrm{~A}$ \\
\hline SA3\#8 & $<1000$ & $1 / 16384 \mathrm{C}$ & SA4\#3 & $<63$ & 1/1024B & SA4\#8 & $63-1000$ & $1 / 1024 \mathrm{D}$ \\
\hline SA3\#8 & $<1000$ & 1/16384D & SA4\#3 & $<63$ & $1 / 1024 \mathrm{C}$ & SA4\#8 & $<1000$ & 1/1024B \\
\hline SA3\#8 & $<63$ & $1 / 64$ & SA4\#3 & $<63$ & $1 / 4096 \mathrm{~A}$ & SA4\#8 & $<1000$ & $1 / 1024 \mathrm{C}$ \\
\hline SA3\#8 & $<63$ & $1 / 256 \mathrm{~A}$ & SA4\#3 & $<63$ & $1 / 4096 \mathrm{~B}$ & SA4\#8 & $<1000$ & $1 / 16384 \mathrm{C}$ \\
\hline SA3\#8 & $<63$ & $1 / 256 \mathrm{~B}$ & SA4\#3 & $<63$ & $1 / 4096 \mathrm{D}$ & SA4\#8 & $<1000$ & $1 / 16384 \mathrm{D}$ \\
\hline SA3\#8 & $<63$ & $1 / 256 \mathrm{C}$ & SA4\#4 & $<1000$ & 1/1024B & SA4\#8 & $<63$ & $1 / 64$ \\
\hline SA3\#8 & $<63$ & $1 / 1024 \mathrm{~B}$ & SA4\#4 & $<1000$ & $1 / 1024 \mathrm{C}$ & SA4\#8 & $<63$ & $1 / 256 \mathrm{~B}$ \\
\hline SA3\#8 & $<63$ & $1 / 1024 \mathrm{C}$ & SA4\#4 & $<1000$ & 1/4096B & SA4\#8 & $<63$ & $1 / 1024 \mathrm{~A}$ \\
\hline SA3\#9 & $<1000$ & $1 / 256 \mathrm{~A}$ & SA4\#4 & $<1000$ & $1 / 16384 C$ & SA4\#8 & $<63$ & 1/1024B \\
\hline SA3\#9 & $<1000$ & $1 / 1024 \mathrm{~A}$ & SA4\#4 & $<1000$ & 1/16384D & $\mathrm{SA} 4 \# 8$ & $<63$ & $1 / 1024 \mathrm{C}$ \\
\hline SA3\#9 & $<1000$ & $1 / 1024 A^{\prime}$ & SA4\#4 & $<63$ & $1 / 64$ & SA4\#8 & $<63$ & $1 / 4096 \mathrm{~A}$ \\
\hline SA3\#9 & $<1000$ & 1/1024B' & SA4\#4 & $<63$ & $1 / 256 \mathrm{~B}$ & SA4\#8 & $<63$ & $1 / 4096 \mathrm{~B}$ \\
\hline SA3\#9 & $<1000$ & 1/16384B & SA4\#4 & $<63$ & $1 / 1024 \mathrm{~A}$ & SA4\#8 & $<63$ & 1/4096D \\
\hline SA3\#9 & $<1000$ & $1 / 16384 C$ & SA4\#4 & $<63$ & 1/1024B & SA4\#9 & $<1000$ & 1/1024B \\
\hline SA3\#9 & $<1000$ & $1 / 16384 \mathrm{D}$ & SA4\#4 & $<63$ & $1 / 1024 \mathrm{C}$ & SA4\#9 & $<1000$ & $1 / 4096 \mathrm{~B}$ \\
\hline SA3\#10 & $<1000$ & $1 / 1024 \mathrm{~A}$ & SA4\#4 & $<63$ & $1 / 4096 \mathrm{~A}$ & SA4\#9 & $<63$ & $1 / 64$ \\
\hline SA3\#10 & $<1000$ & $1 / 1024 A^{\prime}$ & SA4\#4 & $<63$ & $1 / 4096 \mathrm{~B}$ & SA4\#9 & $<63$ & $1 / 256 \mathrm{~B}$ \\
\hline SA3\#10 & $<1000$ & $1 / 1024 \mathrm{~B}$ & SA4\#4 & $<63$ & $1 / 4096 \mathrm{D}$ & SA4\#9 & $<63$ & $1 / 1024 \mathrm{~A}$ \\
\hline SA3\#10 & $<1000$ & $1 / 1024 \mathrm{C}$ & SA4\#5 & $63-1000$ & $1 / 256 \mathrm{~B}$ & SA4\#9 & $<63$ & $1 / 1024 B$ \\
\hline SA3\#10 & $<1000$ & $1 / 4096 \mathrm{C}(5)$ & SA4\#5 & $63-1000$ & $1 / 1024 \mathrm{~B}$ & SA4\#9 & $<63$ & $1 / 1024 \mathrm{C}$ \\
\hline SA3\#10 & $<63$ & $1 / 256 \mathrm{~A}$ & SA4\#5 & $63-1000$ & $1 / 1024 \mathrm{D}$ & SA4\#9 & $<63$ & $1 / 4096 \mathrm{~A}$ \\
\hline SA3\#10 & $<63$ & $1 / 256 \mathrm{C}$ & SA4\#5 & $<1000$ & $1 / 1024 \mathrm{~B}$ & SA4\#9 & $<63$ & $1 / 4096 \mathrm{~A}$ \\
\hline SA3\#11 & $<1000$ & $1 / 256 \mathrm{~A}$ & SA4\#5 & $<1000$ & $1 / 1024 \mathrm{C}$ & SA4\#9 & $<63$ & 1/4096B \\
\hline SA3\#11 & $<1000$ & $1 / 16384 \mathrm{D}$ & SA4\#5 & $<1000$ & 1/4096B & SA4\#9 & $<63$ & 1/4096D \\
\hline SA3\#11 & $<63$ & $1 / 64$ & SA4\#5 & $<1000$ & 1/16384D & SA4\#9 & $<63$ & 1/4096D \\
\hline SA3\#11 & $<63$ & $1 / 256 \mathrm{C}$ & SA4\#5 & $<63$ & $1 / 256 \mathrm{~B}$ & SA4\#10 & $63-1000$ & $1 / 256 \mathrm{~A}$ \\
\hline SA3\#12 & $<63$ & $1 / 256 \mathrm{~B}$ & SA4\#5 & $<63$ & $1 / 1024 \mathrm{~A}$ & SA4\#10 & $<1000$ & $1 / 256 \mathrm{~A}$ \\
\hline SA3\#12 & $<63$ & $1 / 1024 \mathrm{~A}$ & SA4\#5 & $<63$ & 1/1024B & SA4\#10 & $<1000$ & $1 / 256 \mathrm{~B}$ \\
\hline SA4\#1 & $<1000$ & 1/1024B & SA4\#5 & $<63$ & $1 / 1024 \mathrm{C}$ & SA4\#10 & $<1000$ & $1 / 256 \mathrm{C}$ \\
\hline SA4\#1 & $<1000$ & 1/16384B & SA4\#5 & $<63$ & $1 / 4096 \mathrm{~A}$ & SA4\#10 & $<1000$ & $1 / 1024 \mathrm{~B}$ \\
\hline SA4\#1 & $<1000$ & $1 / 16384 C$ & SA4\#5 & $<63$ & $1 / 4096 \mathrm{~B}$ & SA4\#10 & $<1000$ & $1 / 1024 \mathrm{C}$ \\
\hline SA4\#1 & $<1000$ & $1 / 16384 \mathrm{D}$ & SA4\#5 & $<63$ & $1 / 4096 \mathrm{D}$ & SA4\#10 & $<1000$ & 1/4096B \\
\hline SA4\#1 & $<63$ & $1 / 64$ & SA4\#6 & $63-1000$ & $1 / 256 \mathrm{~A}(4)$ & SA4\#10 & $<1000$ & 1/16384B \\
\hline SA4\#1 & $<63$ & $1 / 256 \mathrm{~B}$ & SA4\#6 & $<1000$ & $1 / 1024 \mathrm{~B}$ & SA4\#10 & $<1000$ & $1 / 16384 \mathrm{C}$ \\
\hline SA4\#1 & $<63$ & $1 / 1024 \mathrm{~A}$ & SA4\#6 & $<1000$ & 1/4096B & SA4\#10 & $<1000$ & $1 / 16384 \mathrm{D}$ \\
\hline SA4\#1 & $<63$ & $1 / 1024 \mathrm{~B}$ & SA4\#6 & $<1000$ & 1/16384B & SA4\#10 & $<63$ & $1 / 64$ \\
\hline SA4\#1 & $<63$ & $1 / 1024 \mathrm{C}$ & SA4\#6 & $<1000$ & $1 / 16384 \mathrm{C}$ & SA4\#10 & $<63$ & $1 / 256 \mathrm{~A}$ \\
\hline SA4\#1 & $<63$ & $1 / 4096 \mathrm{~A}$ & SA4\#6 & $<1000$ & $1 / 16384 \mathrm{D}$ & SA4\#10 & $<63$ & $1 / 256 \mathrm{~B}$ \\
\hline SA4\#1 & $<63$ & $1 / 4096 \mathrm{~B}$ & SA4\#6 & $<63$ & $1 / 64$ & SA4\#10 & $<63$ & $1 / 1024 \mathrm{~A}$ \\
\hline SA4\#1 & $<63$ & $1 / 4096 \mathrm{D}$ & SA4\#6 & $<63$ & $1 / 256 \mathrm{~B}$ & SA4\#10 & $<63$ & 1/1024B \\
\hline $\mathrm{SA} 4 \# 2$ & $<1000$ & 1/1024B & SA4\#6 & $<63$ & $1 / 256 \mathrm{C}$ & SA4\#10 & $<63$ & $1 / 1024 \mathrm{C}$ \\
\hline SA4\#2 & $<1000$ & 1/16384B & SA4\#6 & $<63$ & $1 / 1024 \mathrm{~A}$ & SA4\#10 & $<63$ & $1 / 4096 \mathrm{~A}$ \\
\hline SA4\#2 & $<1000$ & $1 / 16384 \mathrm{C}$ & SA4\#6 & $<63$ & $1 / 1024 \mathrm{C}$ & SA4\#10 & $<63$ & 1/4096B \\
\hline SA4\#2 & $<1000$ & 1/16384D & SA4\#6 & $<63$ & $1 / 4096 \mathrm{~A}$ & SA4\#10 & $<63$ & $1 / 4096 \mathrm{D}$ \\
\hline SA4\#2 & $<63$ & $1 / 256 \mathrm{~B}$ & SA4\#6 & $<63$ & 1/4096B & SA4\#11 & $<1000$ & $1 / 1024 \mathrm{~B}$ \\
\hline SA4\#2 & $<63$ & $1 / 1024 \mathrm{~A}$ & SA4\#6 & $<63$ & $1 / 4096 \mathrm{D}$ & SA4\#11 & $<1000$ & $1 / 1024 \mathrm{C}$ \\
\hline SA4\#2 & $<63$ & $1 / 1024 \mathrm{~B}$ & SA4\#7 & $<1000$ & $1 / 1024 \mathrm{~B}$ & SA4\#11 & $<1000$ & $1 / 16384 \mathrm{~A}$ \\
\hline SA4\#2 & $<63$ & $1 / 1024 \mathrm{C}$ & SA4\#7 & $<1000$ & $1 / 1024 \mathrm{C}$ & SA4\#11 & $<1000$ & 1/16384B \\
\hline SA4\#2 & $<63$ & $1 / 4096 \mathrm{~A}$ & SA4\#7 & $<1000$ & 1/4096B & SA4\#11 & $<1000$ & $1 / 16384 \mathrm{C}$ \\
\hline SA4\#2 & $<63$ & 1/4096B & SA4\#7 & $<1000$ & $1 / 16384 C$ & SA4\#11 & $<1000$ & $1 / 16384 \mathrm{D}$ \\
\hline SA4\#2 & $<63$ & $1 / 4096 \mathrm{D}$ & SA4\#7 & $<1000$ & $1 / 16384 \mathrm{D}$ & SA4\#11 & $<63$ & $1 / 64$ \\
\hline SA4\#3 & $<1000$ & $1 / 1024 \mathrm{~B}$ & SA4\#7 & $<63$ & $1 / 256 \mathrm{~B}$ & SA4\#11 & $<63$ & $1 / 256 \mathrm{~B}$ \\
\hline SA4\#3 & $<1000$ & $1 / 4096 \mathrm{C}$ & SA4\#7 & $<63$ & $1 / 256 \mathrm{C}$ & SA4\#11 & $<63$ & $1 / 1024 \mathrm{~A}$ \\
\hline SA4\#3 & $<1000$ & $1 / 16384 \mathrm{C}$ & SA4\#7 & $<63$ & $1 / 1024 \mathrm{~A}$ & SA4\#11 & $<63$ & $1 / 1024 \mathrm{~B}$ \\
\hline
\end{tabular}


Kozo Takahashi, Hirofumi Asahi, Yusuke Okazaki, Jonaotaro Onodera, Hideto Tsutsui,

Takahito Ikenoue, Yoshiyuki Kanematsu, Seiji Tanaka and Shinya Iwasaki

Table 4 (Cont.)

\begin{tabular}{|c|c|c|c|c|c|c|c|c|}
\hline Sample ID & Size $(\mu \mathrm{m})$ & $\begin{array}{l}\text { Aliquot } \\
\text { size \& ID }\end{array}$ & Sample ID & Size $(\mu \mathrm{m})$ & $\begin{array}{l}\text { Aliquot } \\
\text { size \& ID }\end{array}$ & Sample ID & Size $(\mu \mathrm{m})$ & $\begin{array}{l}\text { Aliquot } \\
\text { size \& ID }\end{array}$ \\
\hline SA4\#11 & $<63$ & $1 / 1024 \mathrm{C}$ & $\overline{\text { SA5\#3 }}$ & $<1000$ & $1 / 256 \mathrm{C}$ & $\overline{\text { SA5\#9 }}$ & $<63$ & $1 / 64$ \\
\hline SA4\#11 & $<63$ & $1 / 4096 \mathrm{~A}$ & SA5\#3 & $<1000$ & $1 / 1024 \mathrm{~A}$ & SA5\#9 & $<63$ & $1 / 256 \mathrm{~A}$ \\
\hline SA4\#11 & $<63$ & $1 / 4096 \mathrm{~B}$ & SA5\#3 & $<1000$ & $1 / 1024 \mathrm{~B}$ & SA5\#10 & $<1000$ & $1 / 256 \mathrm{~B}$ \\
\hline SA4\#11 & $<63$ & $1 / 4096 \mathrm{D}$ & SA5\#3 & $<1000$ & 1/1024B & SA5\#10 & $<1000$ & $1 / 256 \mathrm{C}$ \\
\hline SA4\#12 & $<1000$ & 1/1024B & SA5\#3 & $<1000$ & $1 / 1024 \mathrm{C}$ & SA5\#10 & $<1000$ & $1 / 1024 \mathrm{~A}$ \\
\hline SA4\#12 & $<1000$ & $1 / 1024 \mathrm{C}$ & SA5\#3 & $<1000$ & $1 / 4096 \mathrm{~A}$ & SA5\#10 & $<1000$ & $1 / 4096 \mathrm{C}$ \\
\hline SA4\#12 & $<1000$ & $1 / 4096 \mathrm{C}$ & SA5\#3 & $<1000$ & $1 / 4096 \mathrm{~B}$ & SA5\#10 & $<63$ & $1 / 64$ \\
\hline SA4\#12 & $<1000$ & $1 / 16384 \mathrm{~A}$ & SA5\#3 & $<1000$ & $1 / 4096 \mathrm{C}$ & SA5\#11 & $63-1000$ & $1 / 1024 \mathrm{D}$ \\
\hline SA4\#12 & $<1000$ & 1/16384B & SA5\#3 & $<1000$ & $1 / 4096 \mathrm{C}$ & SA6\#1 & $<1000$ & $1 / 1024 \mathrm{C}$ \\
\hline SA4\#12 & $<1000$ & $1 / 16384 \mathrm{C}$ & SA5\#3 & $<1000$ & $1 / 16384 \mathrm{~A}$ & SA6\#1 & $<1000$ & $1 / 4096 \mathrm{~B}$ \\
\hline SA4\#12 & $<1000$ & $1 / 16384 \mathrm{D}$ & SA5\#3 & $<1000$ & 1/16384B & SA6\#1 & $<1000$ & $1 / 16384 \mathrm{~B}$ \\
\hline SA4\#12 & $<63$ & $1 / 256 \mathrm{~B}$ & SA5\#3 & $<1000$ & $1 / 16384 C$ & SA6\#1 & $<1000$ & $1 / 16384 C$ \\
\hline SA4\#12 & $<63$ & $1 / 256 \mathrm{~B}$ & SA5\#3 & $<1000$ & 1/16384D & SA6\#1 & $<1000$ & 1/16384D \\
\hline SA4\#12 & $<63$ & $1 / 256 \mathrm{C}$ & SA5\#3 & $<63$ & $1 / 64$ & SA6\#2 & $<1000$ & $1 / 1024 \mathrm{C}$ \\
\hline SA4\#12 & $<63$ & $1 / 256 \mathrm{C}$ & SA5\#3 & $<63$ & $1 / 256 \mathrm{~A}$ & SA6\#2 & $<1000$ & $1 / 4096 \mathrm{~B}$ \\
\hline SA4\#12 & $<63$ & $1 / 256 \mathrm{D}$ & SA5\#4 & $<1000$ & $1 / 256 \mathrm{~B}$ & SA6\#2 & $<1000$ & 1/16384B \\
\hline SA4\#12 & $<63$ & $1 / 1024 \mathrm{~A}$ & SA5\#4 & $<1000$ & $1 / 256 \mathrm{C}$ & SA6\#2 & $<1000$ & $1 / 16384 \mathrm{C}$ \\
\hline SA4\#12 & $<63$ & $1 / 1024 \mathrm{C}$ & SA5\#4 & $<1000$ & $1 / 1024 \mathrm{~A}$ & SA6\#2 & $<1000$ & 1/16384D \\
\hline SA4\#12 & $<63$ & $1 / 4096 \mathrm{~A}$ & SA5\#4 & $<1000$ & $1 / 4096 \mathrm{C}$ & SA6\#3 & $<1000$ & $1 / 1024 \mathrm{C}$ \\
\hline SA4\#12 & $<63$ & $1 / 4096 \mathrm{C}$ & SA5\#4 & $<63$ & $1 / 64$ & SA6\#3 & $<1000$ & 1/4096B \\
\hline SA4\#12 & $<63$ & $1 / 4096 \mathrm{D}$ & SA5\#4 & $<63$ & $1 / 256 \mathrm{~A}$ & SA6\#3 & $<1000$ & $1 / 16384 \mathrm{~B}$ \\
\hline SA4\#13 & $63-1000$ & $1 / 256 \mathrm{~A}$ & SA5\#5 & $<1000$ & $1 / 256 \mathrm{~A}$ & SA6\#3 & $<1000$ & $1 / 16384 \mathrm{C}$ \\
\hline SA4\#13 & $63-1000$ & $1 / 256 \mathrm{~B}$ & SA5\#5 & $<1000$ & $1 / 256 \mathrm{~B}$ & SA6\#3 & $<1000$ & $1 / 16384 \mathrm{D}$ \\
\hline SA4\#13 & $<1000$ & $1 / 1024 \mathrm{C}$ & SA5\#5 & $<1000$ & $1 / 256 \mathrm{~B}$ & SA6\#4 & $<1000$ & $1 / 1024 \mathrm{C}$ \\
\hline SA4\#13 & $<1000$ & $1 / 1024 \mathrm{~B}$ & SA5\#5 & $<1000$ & $1 / 256 \mathrm{C}$ & SA6\#4 & $<1000$ & $1 / 4096 \mathrm{~B}$ \\
\hline SA4\#13 & $<63$ & $1 / 256 \mathrm{~A}$ & SA5\#5 & $<1000$ & $1 / 256 \mathrm{C}$ & SA6\#4 & $<1000$ & 1/16384B \\
\hline SA4\#13 & $<63$ & $1 / 256 \mathrm{~B}$ & SA5\#5 & $<1000$ & $1 / 1024 \mathrm{~A}$ & SA6\#4 & $<1000$ & $1 / 16384 C$ \\
\hline SA4\#13 & $<63$ & $1 / 256 \mathrm{~B}$ & SA5\#5 & $<1000$ & 1/1024B & SA6\#4 & $<1000$ & 1/16384D \\
\hline SA4\#13 & $<63$ & $1 / 256 \mathrm{C}$ & SA5\#5 & $<1000$ & 1/1024B & SA6\#4 & $<63$ & $2 / 1024$ \\
\hline SA4\#13 & $<63$ & $1 / 256 \mathrm{C}$ & SA5\#5 & $<1000$ & $1 / 1024 \mathrm{C}$ & SA6\#5 & $<1000$ & $1 / 1024 \mathrm{~B}$ \\
\hline SA4\#13 & $<63$ & $1 / 1024 \mathrm{~A}$ & SA5\#5 & $<1000$ & $1 / 4096 \mathrm{~A}$ & SA6\#5 & $<1000$ & $1 / 1024 \mathrm{C}$ \\
\hline SA4\#13 & $<63$ & $1 / 1024 \mathrm{C}$ & SA5\#5 & $<1000$ & 1/4096B & SA6\#5 & $<1000$ & 1/16384B \\
\hline SA4\#13 & $<63$ & $1 / 4096 \mathrm{~A}$ & SA5\#5 & $<1000$ & 1/16384B & SA6\#5 & $<1000$ & $1 / 16384 C$ \\
\hline SA4\#13 & $<63$ & $1 / 4096 \mathrm{C}$ & SA5\#5 & $<1000$ & $1 / 16384 C$ & SA6\#5 & $<1000$ & $1 / 16384 \mathrm{D}$ \\
\hline SA4\#13 & $<63$ & 1/4096D & SA5\#5 & $<1000$ & $1 / 16384 \mathrm{D}$ & SA6\#5 & $<63$ & $1 / 1024$ \\
\hline SA5\#1 & $63-1000$ & $1 / 256 \mathrm{C}(2)$ & SA5\#5 & $<63$ & $1 / 64$ & SA6\#5 & $<63$ & $1 / 1024$ \\
\hline SA5\#1 & $<1000$ & $1 / 256 \mathrm{~A}$ & SA5\#5 & $<63$ & $1 / 256 \mathrm{~A}$ & SA6\#5 & $<63$ & $1 / 1024$ \\
\hline SA5\#1 & $<1000$ & $1 / 256 \mathrm{~B}$ & SA5\#6 & $<1000$ & $1 / 256 \mathrm{~B}$ & SA6\#5 & $<63$ & $2 / 4096$ \\
\hline SA5\#1 & $<1000$ & $1 / 256 \mathrm{C}$ & SA5\#6 & $<1000$ & $1 / 256 \mathrm{C}$ & SA6\#6 & $<1000$ & $1 / 1024 \mathrm{C}$ \\
\hline SA5\#1 & $<1000$ & $1 / 1024 \mathrm{C}$ & SA5\#6 & $<1000$ & $1 / 1024 \mathrm{~A}$ & SA6\#6 & $<1000$ & 1/16384B \\
\hline SA5\#1 & $<1000$ & $1 / 4096 \mathrm{~B}$ & SA5\#6 & $<1000$ & 1/4096B & SA6\#6 & $<1000$ & $1 / 16384 C$ \\
\hline SA5\#1 & $<1000$ & $1 / 4096 \mathrm{C}$ & SA5\#6 & $<63$ & $1 / 64$ & SA6\#6 & $<1000$ & $1 / 16384 \mathrm{D}$ \\
\hline SA5\#1 & $<1000$ & $1 / 16384 \mathrm{~A}$ & SA5\#6 & $<63$ & $1 / 256 \mathrm{~A}$ & SA6\#7 & $<1000$ & $1 / 1024 \mathrm{C}$ \\
\hline SA5\#1 & $<1000$ & 1/16384B & SA5\#7 & $<1000$ & $1 / 256 \mathrm{~B}$ & SA6\#7 & $<1000$ & $1 / 4096 \mathrm{~B}$ \\
\hline SA5\#1 & $<1000$ & $1 / 16384 C$ & SA5\#7 & $<1000$ & $1 / 256 \mathrm{C}$ & SA6\#7 & $<1000$ & 1/16384B \\
\hline SA5\#1 & $<1000$ & $1 / 16384 \mathrm{D}$ & SA5\#7 & $<1000$ & $1 / 1024 \mathrm{~A}$ & SA6\#7 & $<1000$ & $1 / 16384 \mathrm{C}$ \\
\hline SA5\#1 & $<63$ & $1 / 64$ & SA5\#7 & $<1000$ & $1 / 4096 \mathrm{~B}$ & SA6\#7 & $<1000$ & 1/16384D \\
\hline SA5\#1 & $<63$ & $1 / 256 \mathrm{C}$ & SA5\#7 & $<63$ & $1 / 64$ & SA6\#8 & $<1000$ & $1 / 1024 \mathrm{C}$ \\
\hline SA5\#2 & $<1000$ & $1 / 256 \mathrm{~B}$ & SA5\#8 & $<1000$ & $1 / 256 \mathrm{~B}$ & SA6\#8 & $<1000$ & 1/4096B \\
\hline SA5\#2 & $<1000$ & $1 / 256 \mathrm{C}$ & SA5\#8 & $<1000$ & $1 / 256 \mathrm{C}$ & SA6\#8 & $<1000$ & 1/16384B \\
\hline SA5\#2 & $<1000$ & $1 / 1024 \mathrm{~B}$ & SA5\#8 & $<1000$ & $1 / 1024 \mathrm{~B}$ & SA6\#8 & $<1000$ & $1 / 16384 C$ \\
\hline SA5\#2 & $<1000$ & $1 / 4096 \mathrm{C}$ & SA5\#8 & $<1000$ & $1 / 4096 \mathrm{~B}$ & SA6\#8 & $<1000$ & $1 / 16384 \mathrm{D}$ \\
\hline SA5\#2 & $<63$ & $1 / 64$ & SA5\#8 & $<63$ & $1 / 64$ & SA6\#9 & $<1000$ & $1 / 1024 \mathrm{C}$ \\
\hline SA5\#2 & $<63$ & $1 / 256 \mathrm{~A}$ & SA5\#9 & $<1000$ & $1 / 256 \mathrm{~B}$ & SA6\#9 & $<1000$ & $1 / 4096 \mathrm{~B}$ \\
\hline SA5\#3 & $<1000$ & $1 / 256 \mathrm{~A}$ & SA5\#9 & $<1000$ & $1 / 256 \mathrm{C}$ & SA6\#9 & $<1000$ & $1 / 16384 \mathrm{~B}$ \\
\hline SA5\#3 & $<1000$ & $1 / 256 \mathrm{~B}$ & SA5\#9 & $<1000$ & $1 / 1024 \mathrm{~A}$ & SA6\#9 & $<1000$ & $1 / 16384 C$ \\
\hline SA5\#3 & $<1000$ & $1 / 256 \mathrm{C}$ & SA5\#9 & $<1000$ & $1 / 4096 \mathrm{C}$ & SA6\#9 & $<1000$ & $1 / 16384 \mathrm{D}$ \\
\hline
\end{tabular}


Table 4 (Cont.)

\begin{tabular}{|c|c|c|c|c|c|c|c|c|}
\hline Sample ID & Size $(\mu \mathrm{m})$ & $\begin{array}{l}\text { Aliquot } \\
\text { size \& ID }\end{array}$ & Sample ID & Size $(\mu \mathrm{m})$ & $\begin{array}{l}\text { Aliquot } \\
\text { size \& ID }\end{array}$ & Sample ID & Size $(\mu \mathrm{m})$ & $\begin{array}{l}\text { Aliquot } \\
\text { size \& ID }\end{array}$ \\
\hline SA6\#10 & $<1000$ & $1 / 1024 \mathrm{C}$ & $\overline{\text { SA7\#6 }}$ & $63-1000$ & 1/1024B & SA7\#12 & $<63$ & $1 / 256 \mathrm{~B}$ \\
\hline SA6\#10 & $<1000$ & $1 / 4096 \mathrm{C}$ & SA7\#6 & $63-1000$ & 1/4096B & SA7\#12 & $<63$ & 1/4096B \\
\hline SA6\#10 & $<1000$ & 1/16384B & SA7\#6 & $<1000$ & $1 / 1024 \mathrm{~A}$ & SA7\#13 & $63-1000$ & 1/1024B \\
\hline SA6\#10 & $<1000$ & $1 / 16384 \mathrm{C}$ & SA7\#6 & $<1000$ & $1 / 4096 \mathrm{C}$ & SA7\#13 & $63-1000$ & 1/4096B \\
\hline SA6\#10 & $<1000$ & 1/16384D & SA7\#6 & $<1000$ & 1/16384B & SA7\#13 & $<1000$ & $1 / 1024 \mathrm{~A}$ \\
\hline SA6\#11 & $<1000$ & $1 / 1024 \mathrm{C}$ & SA7\#6 & $<1000$ & $1 / 16384 \mathrm{C}$ & SA7\#13 & $<1000$ & $1 / 4096 \mathrm{C}$ \\
\hline SA6\#11 & $<1000$ & $1 / 4096 \mathrm{C}$ & SA7\#6 & $<1000$ & $1 / 16384 \mathrm{D}$ & SA7\#13 & $<1000$ & 1/16384B \\
\hline SA6\#11 & $<1000$ & 1/16384B & SA7\#6 & $<63$ & $1 / 4096 \mathrm{~B}$ & SA7\#13 & $<1000$ & $1 / 16384 \mathrm{C}$ \\
\hline SA6\#11 & $<1000$ & $1 / 16384 \mathrm{C}$ & SA7\#7 & $63-1000$ & 1/1024B & SA7\#13 & $<1000$ & 1/16384D \\
\hline SA6\#11 & $<1000$ & 1/16384D & SA7\#7 & $63-1000$ & 1/4096B & SA7\#13 & $<63$ & $1 / 256 \mathrm{~B}$ \\
\hline SA6\#12 & $<1000$ & $1 / 4096 \mathrm{C}$ & SA7\#7 & $<1000$ & $1 / 1024 \mathrm{~A}$ & SA7\#13 & $<63$ & $1 / 4096 \mathrm{~B}$ \\
\hline SA6\#12 & $<1000$ & 1/16384D & SA7\#7 & $<1000$ & $1 / 4096 \mathrm{C}$ & SA8\#1 & $63-1000$ & 1/1024B \\
\hline SA6\#13 & $<1000$ & $1 / 1024 \mathrm{C}$ & SA7\#7 & $<1000$ & 1/16384B & SA8\#1 & $63-1000$ & 1/4096B \\
\hline SA6\#13 & $<1000$ & 1/16384B & SA7\#7 & $<1000$ & $1 / 16384 \mathrm{C}$ & SA8\#1 & $<1000$ & $1 / 1024 \mathrm{~A}$ \\
\hline SA6\#13 & $<1000$ & $1 / 16384 \mathrm{C}$ & SA7\#7 & $<1000$ & 1/16384D & SA8\#1 & $<1000$ & $1 / 4096 \mathrm{C}$ \\
\hline SA6\#13 & $<1000$ & 1/16384D & SA7\#7 & $<63$ & 1/4096B & SA8\#1 & $<1000$ & 1/16384B \\
\hline SA6\#13 & $<63$ & $2 / 1024$ & SA7\#8 & $63-1000$ & 1/1024B & SA8\#1 & $<1000$ & $1 / 16384 \mathrm{C}$ \\
\hline SA7\#1 & $63-1000$ & 1/1024B & SA7\#8 & $63-1000$ & 1/4096B & SA8\#1 & $<1000$ & 1/16384D \\
\hline SA7\#1 & $63-1000$ & $1 / 4096 \mathrm{~B}$ & SA7\#8 & $<1000$ & $1 / 1024 \mathrm{~A}$ & SA8\#1 & $<63$ & $1 / 256 \mathrm{~B}$ \\
\hline SA7\#1 & $<1000$ & $1 / 1024 \mathrm{~A}$ & SA7\#8 & $<1000$ & $1 / 4096 \mathrm{C}$ & SA8\#1 & $<63$ & 1/4096B \\
\hline SA7\#1 & $<1000$ & $1 / 4096 \mathrm{C}$ & SA7\#8 & $<1000$ & $1 / 16384 \mathrm{~A}$ & SA8\#2 & $63-1000$ & 1/1024B \\
\hline SA7\#1 & $<1000$ & 1/16384B & SA7\#8 & $<1000$ & $1 / 16384 \mathrm{C}$ & SA8\#2 & $63-1000$ & 1/4096B \\
\hline SA7\#1 & $<1000$ & $1 / 16384 \mathrm{C}$ & SA7\#8 & $<1000$ & $1 / 16384 \mathrm{D}$ & SA8\#2 & $<1000$ & $1 / 1024 \mathrm{~A}$ \\
\hline SA7\#1 & $<1000$ & $1 / 16384 \mathrm{D}$ & SA7\#8 & $<63$ & 1/4096B & SA8\#2 & $<1000$ & $1 / 4096 \mathrm{C}$ \\
\hline SA7\#1 & $<63$ & 1/4096B & SA7\#9 & $63-1000$ & $1 / 1024 \mathrm{~B}$ & SA8\#2 & $<1000$ & $1 / 16384 \mathrm{~B}$ \\
\hline $\mathrm{SA} 7 \# 2$ & $63-1000$ & 1/1024B & SA7\#9 & $63-1000$ & $1 / 4096 \mathrm{~B}$ & SA8\#2 & $<1000$ & $1 / 16384 \mathrm{C}$ \\
\hline $\mathrm{SA} 7 \# 2$ & $63-1000$ & 1/4096B & SA7\#9 & $<1000$ & $1 / 1024 \mathrm{~A}$ & SA8\#2 & $<1000$ & $1 / 16384 \mathrm{D}$ \\
\hline SA7\#2 & $<1000$ & $1 / 1024 \mathrm{~A}$ & SA7\#9 & $<1000$ & $1 / 4096 \mathrm{C}$ & SA8\#2 & $<63$ & 1/256B \\
\hline $\mathrm{SA} 7 \# 2$ & $<1000$ & $1 / 4096 \mathrm{C}$ & SA7\#9 & $<1000$ & 1/16384B & SA8\#2 & $<63$ & 1/4096B \\
\hline $\mathrm{SA} 7 \# 2$ & $<1000$ & 1/16384B & SA7\#9 & $<1000$ & $1 / 16384 \mathrm{C}$ & SA8\#3 & $63-1000$ & 1/1024B \\
\hline $\mathrm{SA} 7 \# 2$ & $<1000$ & $1 / 16384 C$ & SA7\#9 & $<1000$ & $1 / 16384 \mathrm{D}$ & SA8\#3 & $63-1000$ & 1/4096B \\
\hline SA7\#2 & $<1000$ & 1/16384D & SA7\#9 & $<63$ & 1/4096B & SA8\#3 & $<1000$ & $1 / 1024 \mathrm{~A}$ \\
\hline $\mathrm{SA} 7 \# 2$ & $<63$ & 1/4096B & $\mathrm{SA} 7 \# 10$ & $63-1000$ & 1/1024B & SA8\#3 & $<1000$ & $1 / 4096 \mathrm{C}$ \\
\hline $\mathrm{SA} 7 \# 3$ & $63-1000$ & $1 / 1024 \mathrm{~B}$ & $\mathrm{SA} 7 \# 10$ & $63-1000$ & $1 / 4096 \mathrm{~B}$ & SA8\#3 & $<1000$ & 1/16384B \\
\hline $\mathrm{SA} 7 \# 3$ & $63-1000$ & $1 / 4096 \mathrm{~B}$ & $\mathrm{SA} 7 \# 10$ & $<1000$ & $1 / 1024 \mathrm{~A}$ & SA8\#3 & $<1000$ & $1 / 16384 C$ \\
\hline SA7\#3 & $<1000$ & $1 / 1024 \mathrm{~A}$ & SA7\#10 & $<1000$ & $1 / 4096 \mathrm{C}$ & SA8\#3 & $<1000$ & 1/16384D \\
\hline $\mathrm{SA} 7 \# 3$ & $<1000$ & $1 / 4096 \mathrm{C}$ & $\mathrm{SA} 7 \# 10$ & $<1000$ & 1/16384B & SA8\#3 & $<63$ & $1 / 256 \mathrm{~B}$ \\
\hline $\mathrm{SA} 7 \# 3$ & $<1000$ & $1 / 16384 \mathrm{~B}$ & $\mathrm{SA} 7 \# 10$ & $<1000$ & $1 / 16384 \mathrm{C}$ & SA8\#3 & $<63$ & 1/4096B \\
\hline SA7\#3 & $<1000$ & $1 / 16384 \mathrm{C}$ & SA7\#10 & $<1000$ & 1/16384D & SA8\#4 & $63-1000$ & 1/1024B \\
\hline SA7\#3 & $<1000$ & 1/16384D & SA7\#10 & $<63$ & $1 / 256 \mathrm{~B}$ & SA8\#4 & $63-1000$ & 1/4096B \\
\hline $\mathrm{SA} 7 \# 3$ & $<63$ & 1/4096B & $\mathrm{SA} 7 \# 10$ & $<63$ & 1/4096B & SA8\#4 & $<1000$ & $1 / 1024 \mathrm{~A}$ \\
\hline $\mathrm{SA} 7 \# 4$ & $63-1000$ & 1/1024B & SA7\#11 & $63-1000$ & 1/1024B & SA8\#4 & $<1000$ & $1 / 4096 \mathrm{C}$ \\
\hline $\mathrm{SA} 7 \# 4$ & $63-1000$ & 1/4096B & SA7\#11 & $63-1000$ & 1/4096B & SA8\#4 & $<1000$ & 1/16384B \\
\hline SA7\#4 & $<1000$ & $1 / 1024 \mathrm{~A}$ & SA7\#11 & $<1000$ & $1 / 1024 \mathrm{~A}$ & SA8\#4 & $<1000$ & $1 / 16384 \mathrm{C}$ \\
\hline $\mathrm{SA} 7 \# 4$ & $<1000$ & $1 / 4096 \mathrm{C}$ & SA7\#11 & $<1000$ & $1 / 4096 \mathrm{C}$ & SA8\#4 & $<1000$ & 1/16384D \\
\hline $\mathrm{SA} 7 \# 4$ & $<1000$ & 1/16384B & SA7\#11 & $<1000$ & 1/16384B & SA8\#4 & $<63$ & $1 / 256 \mathrm{~B}$ \\
\hline $\mathrm{SA} 7 \# 4$ & $<1000$ & $1 / 16384 \mathrm{C}$ & SA7\#11 & $<1000$ & $1 / 16384 \mathrm{C}$ & SA8\#4 & $<63$ & 1/4096B \\
\hline SA7\#4 & $<1000$ & $1 / 16384 \mathrm{D}$ & SA7\#11 & $<1000$ & 1/16384D & SA8\#5 & $63-1000$ & $1 / 1024 \mathrm{~B}$ \\
\hline $\mathrm{SA} 7 \# 4$ & $<63$ & 1/4096B & SA7\#11 & $<63$ & $1 / 256 \mathrm{~B}$ & SA8\#5 & $63-1000$ & 1/4096B \\
\hline SA7\#5 & $63-1000$ & $1 / 1024 \mathrm{~B}$ & SA7\#11 & $<63$ & $1 / 4096 \mathrm{~B}$ & SA8\#5 & $<1000$ & $1 / 1024 \mathrm{~A}$ \\
\hline $\mathrm{SA} 7 \# 5$ & $63-1000$ & $1 / 4096 \mathrm{C}$ & SA7\#12 & $63-1000$ & 1/1024B & SA8\#5 & $<1000$ & $1 / 4096 \mathrm{C}$ \\
\hline $\mathrm{SA} 7 \# 5$ & $<1000$ & $1 / 1024 \mathrm{~A}$ & SA7\#12 & $63-1000$ & $1 / 4096 \mathrm{~B}$ & SA8\#5 & $<1000$ & $1 / 16384 \mathrm{~B}$ \\
\hline $\mathrm{SA} 7 \# 5$ & $<1000$ & $1 / 4096 \mathrm{C}$ & $\mathrm{SA} 7 \# 12$ & $<1000$ & $1 / 1024 \mathrm{~A}$ & SA8\#5 & $<1000$ & $1 / 16384 \mathrm{C}$ \\
\hline SA7\#5 & $<1000$ & $1 / 16384 \mathrm{~B}$ & SA7\#12 & $<1000$ & $1 / 4096 \mathrm{C}$ & SA8\#5 & $<1000$ & $1 / 16384 \mathrm{D}$ \\
\hline SA7\#5 & $<1000$ & $1 / 16384 \mathrm{C}$ & SA7\#12 & $<1000$ & 1/16384B & SA8\#5 & $<63$ & $1 / 256 \mathrm{~B}$ \\
\hline $\mathrm{SA} 7 \# 5$ & $<1000$ & $1 / 16384 \mathrm{D}$ & $\mathrm{SA} 7 \# 12$ & $<1000$ & $1 / 16384 \mathrm{C}$ & SA8\#5 & $<63$ & $1 / 4096 \mathrm{~B}$ \\
\hline SA7\#5 & $<63$ & 1/4096B & SA7\#12 & $<1000$ & $1 / 16384 \mathrm{D}$ & SA8\#6 & $63-1000$ & 1/1024B \\
\hline
\end{tabular}


Kozo Takahashi, Hirofumi Asahi, Yusuke Okazaki, Jonaotaro Onodera, Hideto Tsutsui,

Takahito Ikenoue, Yoshiyuki Kanematsu, Seiji Tanaka and Shinya Iwasaki

Table 4 (Cont.)

\begin{tabular}{|c|c|c|c|c|c|c|c|c|}
\hline Sample ID & Size $(\mu \mathrm{m})$ & $\begin{array}{l}\text { Aliquot } \\
\text { size \& ID }\end{array}$ & Sample ID & Size $(\mu \mathrm{m})$ & $\begin{array}{l}\text { Aliquot } \\
\text { size \& ID }\end{array}$ & Sample ID & Size $(\mu \mathrm{m})$ & $\begin{array}{l}\text { Aliquot } \\
\text { size \& ID }\end{array}$ \\
\hline SA8\#6 & $63-1000$ & 1/4096B & SA8\#12 & $<1000$ & 1/16384B & SA9\#5 & $<63$ & $1 / 1024 \mathrm{~B}$ \\
\hline SA8\#6 & $<1000$ & $1 / 1024 \mathrm{~A}$ & SA8\#12 & $<1000$ & $1 / 16384 \mathrm{C}$ & SA9\#6 & 63-1000 & 1/1024B \\
\hline SA8\#6 & $<1000$ & $1 / 4096 \mathrm{C}$ & SA8\#12 & $<1000$ & $1 / 16384 \mathrm{D}$ & SA9\#6 & $63-1000$ & $1 / 4096 \mathrm{~B}$ \\
\hline SA8\#6 & $<1000$ & 1/16384B & SA8\#12 & $<63$ & $1 / 256 \mathrm{~B}$ & SA9\#6 & $<1000$ & $1 / 1024 \mathrm{~A}$ \\
\hline SA8\#6 & $<1000$ & $1 / 16384 \mathrm{C}$ & SA8\#12 & $<63$ & 1/4096B & SA9\#6 & $<1000$ & $1 / 4096 \mathrm{C}$ \\
\hline SA8\#6 & $<1000$ & $1 / 16384 \mathrm{D}$ & SA8\#13 & $63-1000$ & 1/1024B & SA9\#6 & $<1000$ & 1/16384B \\
\hline SA8\#6 & $<63$ & $1 / 256 \mathrm{~B}$ & SA8\#13 & $63-1000$ & 1/4096B & SA9\#6 & $<1000$ & $1 / 16384 \mathrm{C}$ \\
\hline SA8\#6 & $<63$ & 1/4096B & SA8\#13 & $<1000$ & $1 / 1024 \mathrm{~A}$ & SA9\#6 & $<1000$ & 1/16384D \\
\hline SA8\#7 & $63-1000$ & 1/1024B & SA8\#13 & $<1000$ & $1 / 4096 \mathrm{C}$ & SA9\#6 & $<63$ & $1 / 256 \mathrm{C}$ \\
\hline SA8\#7 & $63-1000$ & $1 / 4096 \mathrm{~B}$ & SA8\#13 & $<1000$ & 1/16384B & SA9\#6 & $<63$ & $1 / 4096 \mathrm{~B}$ \\
\hline SA8\#7 & $<1000$ & $1 / 1024 \mathrm{~A}$ & SA8\#13 & $<1000$ & $1 / 16384 \mathrm{C}$ & SA9\#7 & $63-1000$ & 1/1024B \\
\hline SA8\#7 & $<1000$ & $1 / 4096 \mathrm{C}$ & SA8\#13 & $<1000$ & 1/16384D & SA9\#7 & $63-1000$ & 1/4096B \\
\hline SA8\#7 & $<1000$ & 1/16384B & SA8\#13 & $<63$ & $1 / 256 \mathrm{~B}$ & SA9\#7 & $<1000$ & $1 / 1024 \mathrm{~A}$ \\
\hline SA8\#7 & $<1000$ & $1 / 16384 \mathrm{C}$ & SA8\#13 & $<63$ & $1 / 4096 \mathrm{~B}$ & SA9\#7 & $<1000$ & $1 / 4096 \mathrm{C}$ \\
\hline SA8\#7 & $<1000$ & $1 / 16384 \mathrm{D}$ & SA9\#1 & $63-1000$ & 1/1024B & SA9\#7 & $<1000$ & 1/16384B \\
\hline SA8\#7 & $<63$ & $1 / 256 \mathrm{~B}$ & SA9\#1 & $63-1000$ & 1/4096B & SA9\#7 & $<1000$ & $1 / 16384 \mathrm{C}$ \\
\hline SA8\#7 & $<63$ & 1/4096B & SA9\#1 & $<1000$ & $1 / 1024 \mathrm{~A}$ & SA9\#7 & $<1000$ & 1/16384D \\
\hline SA8\#8 & $63-1000$ & $1 / 1024 \mathrm{~B}$ & SA9\#1 & $<1000$ & $1 / 4096 \mathrm{C}$ & SA9\#7 & $<63$ & $1 / 256 \mathrm{C}$ \\
\hline SA8\#8 & $63-1000$ & $1 / 4096 \mathrm{~B}$ & SA9\#1 & $<1000$ & 1/16384B & SA9\#7 & $<63$ & 1/1024B \\
\hline SA8\#8 & $<1000$ & $1 / 1024 \mathrm{~A}$ & SA9\#1 & $<1000$ & $1 / 16384 \mathrm{C}$ & SA9\#8 & $63-1000$ & 1/1024B \\
\hline SA8\#8 & $<1000$ & $1 / 4096 \mathrm{C}$ & SA9\#1 & $<1000$ & $1 / 16384 \mathrm{D}$ & SA9\#8 & $63-1000$ & 1/4096B \\
\hline SA8\#8 & $<1000$ & 1/16384B & SA9\#1 & $<63$ & $1 / 256 \mathrm{C}$ & SA9\#8 & $<1000$ & $1 / 1024 \mathrm{~A}$ \\
\hline SA8\#8 & $<1000$ & $1 / 16384 \mathrm{C}$ & SA9\#1 & $<63$ & 1/4096B & SA9\#8 & $<1000$ & $1 / 4096 \mathrm{C}$ \\
\hline SA8\#8 & $<1000$ & $1 / 16384 \mathrm{D}$ & SA9\#2 & $63-1000$ & $1 / 1024 \mathrm{~B}$ & SA9\#8 & $<1000$ & 1/16384B \\
\hline SA8\#8 & $<63$ & $1 / 256 \mathrm{~B}$ & SA9\#2 & $63-1000$ & $1 / 4096 \mathrm{~B}$ & SA9\#8 & $<1000$ & $1 / 16384 \mathrm{C}$ \\
\hline SA8\#8 & $<63$ & $1 / 4096 \mathrm{~B}$ & SA9\#2 & $<1000$ & $1 / 1024 \mathrm{~A}$ & SA9\#8 & $<1000$ & 1/16384D \\
\hline SA8\#9 & $63-1000$ & 1/1024B & SA9\#2 & $<1000$ & $1 / 4096 \mathrm{C}$ & SA9\#8 & $<63$ & $1 / 256 \mathrm{C}$ \\
\hline SA8\#9 & $63-1000$ & 1/4096B & SA9\#2 & $<1000$ & 1/16384B & SA9\#8 & $<63$ & 1/1024B \\
\hline SA8\#9 & $<1000$ & $1 / 1024 \mathrm{~A}$ & SA9\#2 & $<1000$ & $1 / 16384 C$ & SA9\#9 & $63-1000$ & 1/1024B \\
\hline SA8\#9 & $<1000$ & $1 / 4096 \mathrm{C}$ & SA9\#2 & $<1000$ & $1 / 16384 \mathrm{D}$ & SA9\#9 & $63-1000$ & 1/4096B \\
\hline SA8\#9 & $<1000$ & 1/16384B & SA9\#2 & $<63$ & $1 / 256 \mathrm{C}$ & SA9\#9 & $<1000$ & $1 / 1024 \mathrm{~A}$ \\
\hline SA8\#9 & $<1000$ & $1 / 16384 \mathrm{C}$ & SA9\#2 & $<63$ & 1/4096B & SA9\#9 & $<1000$ & $1 / 4096 \mathrm{C}$ \\
\hline SA8\#9 & $<1000$ & 1/16384D & SA9\#3 & $63-1000$ & 1/1024B & SA9\#9 & $<1000$ & 1/16384B \\
\hline SA8\#9 & $<63$ & $1 / 256 \mathrm{~B}$ & SA9\#3 & $63-1000$ & $1 / 4096 \mathrm{~B}$ & SA9\#9 & $<1000$ & $1 / 16384 \mathrm{C}$ \\
\hline SA8\#9 & $<63$ & 1/4096B & SA9\#3 & $<1000$ & $1 / 1024 \mathrm{~A}$ & SA9\#9 & $<1000$ & 1/16384D \\
\hline SA8\#10 & 63-1000 & 1/1024B & SA9\#3 & $<1000$ & $1 / 4096 \mathrm{C}$ & SA9\#9 & $<63$ & $1 / 256 \mathrm{C}$ \\
\hline SA8\#10 & $63-1000$ & $1 / 4096 \mathrm{~B}$ & SA9\#3 & $<1000$ & 1/16384B & SA9\#9 & $<63$ & 1/1024B \\
\hline SA8\#10 & $<1000$ & $1 / 1024 \mathrm{~A}$ & SA9\#3 & $<1000$ & $1 / 16384 C$ & SA9\#10 & $63-1000$ & 1/1024B \\
\hline SA8\#10 & $<1000$ & $1 / 4096 \mathrm{C}$ & SA9\#3 & $<1000$ & $1 / 16384 \mathrm{D}$ & SA9\#10 & $63-1000$ & $1 / 1024 \mathrm{C}$ \\
\hline SA8\#10 & $<1000$ & 1/16384B & SA9\#3 & $<63$ & $1 / 256 \mathrm{C}$ & SA9\#10 & $63-1000$ & 1/4096B \\
\hline SA8\#10 & $<1000$ & $1 / 16384 \mathrm{C}$ & SA9\#3 & $<63$ & 1/4096B & SA9\#10 & $63-1000$ & $1 / 4096 \mathrm{C}$ \\
\hline SA8\#10 & $<1000$ & $1 / 16384 \mathrm{D}$ & SA9\#4 & $63-1000$ & 1/1024B & SA9\#10 & $<1000$ & $1 / 1024 \mathrm{~A}$ \\
\hline SA8\#10 & $<63$ & $1 / 256 \mathrm{~B}$ & SA9\#4 & $63-1000$ & 1/4096B & SA9\#10 & $<1000$ & 1/16384B \\
\hline SA8\#10 & $<63$ & 1/4096B & SA9\#4 & $<1000$ & $1 / 1024 \mathrm{~A}$ & SA9\#10 & $<1000$ & $1 / 16384 \mathrm{C}$ \\
\hline SA8\#11 & $63-1000$ & $1 / 1024 \mathrm{~B}$ & SA9\#4 & $<1000$ & $1 / 4096 \mathrm{C}$ & SA9\#10 & $<1000$ & 1/16384D \\
\hline SA8\#11 & $63-1000$ & $1 / 4096 \mathrm{~B}$ & SA9\#4 & $<1000$ & 1/16384B & SA9\#10 & $<63$ & $1 / 256 \mathrm{C}$ \\
\hline SA8\#11 & $<1000$ & $1 / 1024 \mathrm{~A}$ & SA9\#4 & $<1000$ & $1 / 16384 \mathrm{C}$ & SA9\#10 & $<63$ & 1/1024B \\
\hline SA8\#11 & $<1000$ & $1 / 4096 \mathrm{C}$ & SA9\#4 & $<1000$ & $1 / 16384 \mathrm{D}$ & SA9\#10 & $<63$ & $1 / 1024 \mathrm{C}$ \\
\hline SA8\#11 & $<1000$ & 1/16384B & SA9\#4 & $<63$ & 1/4096B & SA9\#11 & $63-1000$ & $1 / 1024 \mathrm{~B}$ \\
\hline SA8\#11 & $<1000$ & $1 / 16384 \mathrm{C}$ & SA9\#5 & $63-1000$ & 1/1024B & SA9\#11 & $63-1000$ & $1 / 4096 \mathrm{~B}$ \\
\hline SA8\#11 & $<1000$ & 1/16384D & SA9\#5 & $63-1000$ & 1/4096B & SA9\#11 & $<1000$ & $1 / 1024 \mathrm{~A}$ \\
\hline SA8\#11 & $<63$ & $1 / 256 \mathrm{~B}$ & SA9\#5 & $<1000$ & $1 / 1024 \mathrm{~A}$ & SA9\#11 & $<1000$ & $1 / 4096 \mathrm{C}$ \\
\hline SA8\#11 & $<63$ & $1 / 4096 \mathrm{~B}$ & SA9\#5 & $<1000$ & $1 / 4096 \mathrm{C}$ & SA9\#11 & $<1000$ & 1/16384B \\
\hline SA8\#12 & $63-1000$ & $1 / 1024 \mathrm{~B}$ & SA9\#5 & $<1000$ & 1/16384B & SA9\#11 & $<1000$ & $1 / 16384 \mathrm{C}$ \\
\hline SA8\#12 & $63-1000$ & 1/4096B & SA9\#5 & $<1000$ & $1 / 16384 \mathrm{C}$ & SA9\#11 & $<1000$ & 1/16384D \\
\hline SA8\#12 & $<1000$ & $1 / 1024 \mathrm{~A}$ & SA9\#5 & $<1000$ & $1 / 16384 \mathrm{D}$ & SA9\#11 & $<63$ & $1 / 256 \mathrm{C}$ \\
\hline SA8\#12 & $<1000$ & $1 / 4096 \mathrm{C}$ & SA9\#5 & $<63$ & $1 / 256 \mathrm{C}$ & SA9\#11 & $<63$ & 1/1024B \\
\hline
\end{tabular}


Table 4 (Cont.)

\begin{tabular}{|c|c|c|c|c|c|c|c|c|}
\hline Sample ID & Size $(\mu \mathrm{m})$ & $\begin{array}{l}\text { Aliquot } \\
\text { size \& ID }\end{array}$ & Sample ID & Size $(\mu \mathrm{m})$ & $\begin{array}{l}\text { Aliquot } \\
\text { size \& ID }\end{array}$ & Sample ID & Size $(\mu \mathrm{m})$ & $\begin{array}{l}\text { Aliquot } \\
\text { size \& ID }\end{array}$ \\
\hline SA9\#12 & $63-1000$ & 1/1024B & SA10\#4 & $63-1000$ & $1 / 1024 \mathrm{C}$ & SA10\#9 & $63-1000$ & $1 / 4096 \mathrm{C}$ \\
\hline SA9\#12 & $63-1000$ & 1/4096B & SA10\#4 & $63-1000$ & $1 / 4096 \mathrm{C}$ & SA10\#9 & $<1000$ & $1 / 4096 \mathrm{~A}$ \\
\hline SA9\#12 & $<1000$ & $1 / 1024 \mathrm{~A}$ & SA10\#4 & $<1000$ & $1 / 4096 \mathrm{~A}$ & SA10\#9 & $<1000$ & $1 / 4096 \mathrm{~B}$ \\
\hline SA9\#12 & $<1000$ & $1 / 4096 \mathrm{C}$ & SA10\#4 & $<1000$ & 1/4096B & SA10\#9 & $<1000$ & $1 / 16384 \mathrm{~A}$ \\
\hline SA9\#12 & $<1000$ & 1/16384B & SA10\#4 & $<1000$ & $1 / 16384 \mathrm{~A}$ & SA10\#9 & $<1000$ & 1/16384B \\
\hline SA9\#12 & $<1000$ & $1 / 16384 \mathrm{C}$ & SA10\#4 & $<1000$ & 1/16384B & SA10\#9 & $<1000$ & $1 / 16384 C$ \\
\hline SA9\#12 & $<1000$ & $1 / 16384 \mathrm{D}$ & SA10\#4 & $<1000$ & $1 / 16384 \mathrm{C}$ & SA10\#9 & $<1000$ & $1 / 16384 \mathrm{D}$ \\
\hline SA9\#12 & $<63$ & $1 / 256 \mathrm{C}$ & SA10\#4 & $<1000$ & 1/16384D & SA10\#10 & $63-1000$ & $1 / 1024 \mathrm{C}$ \\
\hline SA9\#12 & $<63$ & 1/1024B & SA10\#5 & $63-1000$ & $1 / 1024 \mathrm{C}$ & SA10\#10 & $63-1000$ & $1 / 4096 \mathrm{C}$ \\
\hline SA9\#13 & $63-1000$ & $1 / 1024 \mathrm{C}$ & SA10\#5 & $63-1000$ & $1 / 4096 \mathrm{C}$ & SA10\#10 & $63-1000$ & $1 / 4096 \mathrm{C}$ \\
\hline SA9\#13 & $63-1000$ & $1 / 4096 \mathrm{~B}$ & SA10\#5 & $<1000$ & $1 / 4096 \mathrm{~A}$ & SA10\#10 & $<1000$ & $1 / 4096 \mathrm{~A}$ \\
\hline SA9\#13 & $<1000$ & $1 / 1024 \mathrm{~A}$ & SA10\#5 & $<1000$ & 1/4096B & SA10\#10 & $<1000$ & 1/4096B \\
\hline SA9\#13 & $<1000$ & $1 / 4096 \mathrm{C}$ & SA10\#5 & $<1000$ & $1 / 16384 \mathrm{~A}$ & SA10\#10 & $<1000$ & $1 / 16384 \mathrm{~A}$ \\
\hline SA9\#13 & $<1000$ & 1/16384B & SA10\#5 & $<1000$ & 1/16384B & SA10\#10 & $<1000$ & 1/16384B \\
\hline SA9\#13 & $<1000$ & $1 / 16384 \mathrm{C}$ & SA10\#5 & $<1000$ & $1 / 16384 \mathrm{C}$ & SA10\#10 & $<1000$ & $1 / 16384 \mathrm{C}$ \\
\hline SA9\#13 & $<1000$ & 1/16384D & SA10\#5 & $<1000$ & 1/16384D & SA10\#10 & $<1000$ & $1 / 16384 \mathrm{D}$ \\
\hline SA9\#13 & $<63$ & $1 / 256 \mathrm{C}$ & SA10\#6 & $63-1000$ & $1 / 1024 \mathrm{C}$ & SA10\#11 & $63-1000$ & $1 / 1024 \mathrm{C}$ \\
\hline SA9\#13 & $<63$ & $1 / 1024 \mathrm{~B}$ & SA10\#6 & $63-1000$ & $1 / 4096 \mathrm{C}$ & SA10\#11 & $63-1000$ & $1 / 4096 \mathrm{C}$ \\
\hline SA10\#1 & $63-1000$ & $1 / 1024 \mathrm{C}$ & SA10\#6 & $<1000$ & $1 / 4096 \mathrm{~A}$ & SA10\#11 & $<1000$ & $1 / 4096 \mathrm{~A}$ \\
\hline SA10\#1 & $63-1000$ & $1 / 4096 \mathrm{C}$ & SA10\#6 & $<1000$ & 1/4096B & SA10\#11 & $<1000$ & 1/4096B \\
\hline SA10\#1 & $<1000$ & $1 / 4096 \mathrm{~A}$ & SA10\#6 & $<1000$ & $1 / 16384 \mathrm{~A}$ & SA10\#11 & $<1000$ & $1 / 16384 \mathrm{~A}$ \\
\hline SA10\#1 & $<1000$ & 1/4096B & SA10\#6 & $<1000$ & 1/16384B & SA10\#11 & $<1000$ & 1/16384B \\
\hline SA10\#1 & $<1000$ & $1 / 16384 \mathrm{~A}$ & SA10\#6 & $<1000$ & $1 / 16384 \mathrm{C}$ & SA10\#11 & $<1000$ & $1 / 16384 \mathrm{C}$ \\
\hline SA10\#1 & $<1000$ & 1/16384B & SA10\#6 & $<1000$ & $1 / 16384 \mathrm{D}$ & SA10\#11 & $<1000$ & 1/16384D \\
\hline SA10\#1 & $<1000$ & $1 / 16384 \mathrm{C}$ & SA10\#7 & $63-1000$ & $1 / 1024 \mathrm{C}$ & SA10\#12 & $63-1000$ & $1 / 1024 \mathrm{C}$ \\
\hline SA10\#1 & $<1000$ & $1 / 16384 \mathrm{D}$ & SA10\#7 & $63-1000$ & $1 / 4096 \mathrm{C}$ & SA10\#12 & $63-1000$ & $1 / 4096 \mathrm{C}$ \\
\hline SA10\#2 & $63-1000$ & $1 / 4096 \mathrm{C}$ & SA10\#7 & $<1000$ & $1 / 4096 \mathrm{~A}$ & SA10\#12 & $<1000$ & $1 / 4096 \mathrm{~A}$ \\
\hline SA10\#2 & $<1000$ & $1 / 4096 \mathrm{~A}$ & SA10\#7 & $<1000$ & 1/4096B & SA10\#12 & $<1000$ & 1/4096B \\
\hline SA10\#2 & $<1000$ & 1/4096B & SA10\#7 & $<1000$ & $1 / 16384 \mathrm{~A}$ & SA10\#12 & $<1000$ & $1 / 16384 \mathrm{~A}$ \\
\hline SA10\#2 & $<1000$ & $1 / 16384 \mathrm{~A}$ & SA10\#7 & $<1000$ & 1/16384B & SA10\#12 & $<1000$ & 1/16384B \\
\hline SA10\#2 & $<1000$ & 1/16384B & SA10\#7 & $<1000$ & $1 / 16384 \mathrm{C}$ & SA10\#12 & $<1000$ & $1 / 16384 \mathrm{C}$ \\
\hline SA10\#2 & $<1000$ & $1 / 16384 \mathrm{C}$ & SA10\#7 & $<1000$ & 1/16384D & SA10\#12 & $<1000$ & 1/16384D \\
\hline SA10\#2 & $<1000$ & $1 / 16384 \mathrm{D}$ & SA10\#8 & $63-1000$ & $1 / 1024 \mathrm{C}$ & SA10\#13 & $63-1000$ & $1 / 1024 \mathrm{C}$ \\
\hline SA10\#2 & $<63$ & $1 / 1024 \mathrm{C}$ & SA10\#8 & $63-1000$ & $1 / 4096 \mathrm{C}$ & SA10\#13 & $63-1000$ & $1 / 4096 \mathrm{C}$ \\
\hline SA10\#3 & $63-1000$ & $1 / 1024 \mathrm{C}$ & SA10\#8 & $<1000$ & $1 / 4096 \mathrm{~A}$ & SA10\#13 & $<1000$ & $1 / 4096 \mathrm{~A}$ \\
\hline SA10\#3 & $<1000$ & $1 / 4096 \mathrm{~A}$ & SA10\#8 & $<1000$ & 1/4096B & SA10\#13 & $<1000$ & 1/4096B \\
\hline SA10\#3 & $<1000$ & 1/4096B & SA10\#8 & $<1000$ & $1 / 16384 \mathrm{~A}$ & SA10\#13 & $<1000$ & $1 / 16384 \mathrm{~A}$ \\
\hline SA10\#3 & $<1000$ & $1 / 16384 \mathrm{~A}$ & SA10\#8 & $<1000$ & 1/16384B & SA10\#13 & $<1000$ & $1 / 16384 \mathrm{~B}$ \\
\hline SA10\#3 & $<1000$ & 1/16384B & SA10\#8 & $<1000$ & $1 / 16384 C$ & SA10\#13 & $<1000$ & $1 / 16384 C$ \\
\hline SA10\#3 & $<1000$ & $1 / 16384 \mathrm{C}$ & SA10\#8 & $<1000$ & $1 / 16384 \mathrm{D}$ & SA10\#13 & $<1000$ & 1/16384D \\
\hline SA10\#3 & $<1000$ & $1 / 16384 \mathrm{D}$ & SA10\#9 & $63-1000$ & $1 / 1024 \mathrm{C}$ & & & \\
\hline
\end{tabular}

Table 5. List of archival filter samples from Station AB.

\begin{tabular}{|c|c|c|c|c|c|c|c|c|}
\hline Sample ID & Size $(\mu \mathrm{m})$ & $\begin{array}{l}\text { Aliquot } \\
\text { size \& ID }\end{array}$ & Sample ID & Size $(\mu \mathrm{m})$ & $\begin{array}{l}\text { Aliquot } \\
\text { size \& ID }\end{array}$ & Sample ID & Size $(\mu \mathrm{m})$ & $\begin{array}{l}\text { Aliquot } \\
\text { size \& ID }\end{array}$ \\
\hline$\overline{\mathrm{AB} 1 \# 1}$ & $63-1000$ & $1 / 256 a$ & $\overline{\mathrm{AB} 1 \# 1}$ & $<63$ & $1 / 1024$ & $\overline{\mathrm{AB} 1 \# 2}$ & $63-1000$ & $1 / 4096 \mathrm{D}$ \\
\hline $\mathrm{AB} 1 \# 1$ & $63-1000$ & $1 / 256 b$ & $\mathrm{AB} 1 \# 1$ & $<63$ & $1 / 1024 \mathrm{~A}$ & $\mathrm{AB} 1 \# 2$ & $<1000$ & $1 / 1024 \mathrm{~A}$ \\
\hline $\mathrm{AB} 1 \# 1$ & $63-1000$ & $1 / 1024 \mathrm{C}$ & $\mathrm{AB} 1 \# 1$ & $<63$ & $1 / 4096$ & $\mathrm{AB} 1 \# 2$ & $<1000$ & 1/1024B \\
\hline $\mathrm{AB} 1 \# 1$ & $63-1000$ & $1 / 4096 \mathrm{~A}$ & $\mathrm{AB} 1 \# 1$ & $<63$ & $1 / 4096$ & $\mathrm{AB} 1 \# 2$ & $<1000$ & 1/16384B \\
\hline $\mathrm{AB} 1 \# 1$ & $63-1000$ & $1 / 4096 \mathrm{C}$ & $\mathrm{AB} 1 \# 1$ & $<63$ & 1/4096A & $\mathrm{AB} 1 \# 2$ & $<1000$ & $1 / 16384 \mathrm{C}$ \\
\hline $\mathrm{AB} 1 \# 1$ & 63-1000 & $1 / 4096 \mathrm{D}$ & $\mathrm{AB} 1 \# 2$ & $63-1000$ & $1 / 64$ & $\mathrm{AB} 1 \# 2$ & $<1000$ & 1/16384D \\
\hline $\mathrm{AB} 1 \# 1$ & $<1000$ & 1/1024B & $\mathrm{AB} 1 \# 2$ & $63-1000$ & $1 / 256 a$ & $\mathrm{AB} 1 \# 2$ & $<63$ & $1 / 256 a$ \\
\hline $\mathrm{AB} 1 \# 1$ & $<1000$ & 1/16384B & $\mathrm{AB} 1 \# 2$ & $63-1000$ & $1 / 1024 \mathrm{~A}$ & $\mathrm{AB} 1 \# 2$ & $<63$ & $1 / 256 b$ \\
\hline $\mathrm{AB} 1 \# 1$ & $<1000$ & $1 / 16384 \mathrm{C}$ & $\mathrm{AB} 1 \# 2$ & $63-1000$ & 1/4096A & $\mathrm{AB} 1 \# 2$ & $<63$ & $1 / 1024$ \\
\hline $\mathrm{AB} 1 \# 1$ & $<1000$ & 1/16384D & $\mathrm{AB} 1 \# 2$ & $63-1000$ & $1 / 4096 \mathrm{C}$ & $\mathrm{AB} 1 \# 2$ & $<63$ & $1 / 1024 \mathrm{~A}$ \\
\hline
\end{tabular}


Kozo Takahashi, Hirofumi Asahi, Yusuke Okazaki, Jonaotaro Onodera, Hideto Tsutsui,

Takahito Ikenoue, Yoshiyuki Kanematsu, Seiji Tanaka and Shinya Iwasaki

Table 5 (Cont.)

\begin{tabular}{|c|c|c|c|c|c|c|c|c|}
\hline Sample ID & Size $(\mu \mathrm{m})$ & $\begin{array}{l}\text { Aliquot } \\
\text { size \& ID }\end{array}$ & Sample ID & Size $(\mu \mathrm{m})$ & $\begin{array}{l}\text { Aliquot } \\
\text { size \& ID }\end{array}$ & Sample ID & Size $(\mu \mathrm{m})$ & $\begin{array}{l}\text { Aliquot } \\
\text { size \& ID }\end{array}$ \\
\hline $\mathrm{AB} 1 \# 2$ & $<63$ & $1 / 4096$ & $\overline{\mathrm{AB} 1 \# 6}$ & $63-1000$ & $1 / 256 b$ & $\overline{\mathrm{AB} 1 \# 8}$ & $<1000$ & 1/4096B \\
\hline $\mathrm{AB} 1 \# 2$ & $<63$ & $1 / 4096$ & AB1\#6 & $63-1000$ & $1 / 1024 \mathrm{C}$ & $\mathrm{AB} 1 \# 8$ & $<63$ & $1 / 64$ \\
\hline $\mathrm{AB} 1 \# 2$ & $<63$ & $1 / 4096 \mathrm{~A}$ & AB1\#6 & $63-1000$ & $1 / 4096 \mathrm{~A}$ & $\mathrm{AB} 1 \# 8$ & $<63$ & $1 / 256 a$ \\
\hline $\mathrm{AB} 1 \# 3$ & $63-1000$ & $1 / 64$ & $\mathrm{AB} 1 \# 6$ & $63-1000$ & $1 / 4096 \mathrm{~B}$ & $\mathrm{AB} 1 \# 8$ & $<63$ & $1 / 1024 \mathrm{~A}$ \\
\hline $\mathrm{AB} 1 \# 3$ & $63-1000$ & $1 / 256 a$ & AB1\#6 & $63-1000$ & $1 / 4096 \mathrm{D}$ & AB1\#8 & $<63$ & $1 / 4096$ \\
\hline $\mathrm{AB} 1 \# 3$ & $63-1000$ & $1 / 256 b$ & AB1\#6 & $<1000$ & $1 / 1024 \mathrm{~A}$ & $\mathrm{AB} 1 \# 8$ & $<63$ & $1 / 4096$ \\
\hline $\mathrm{AB} 1 \# 3$ & $63-1000$ & $1 / 1024 \mathrm{~A}$ & AB1\#6 & $<1000$ & $1 / 1024 \mathrm{~B}$ & $\mathrm{AB} 1 \# 8$ & $<63$ & $1 / 4096 \mathrm{~A}$ \\
\hline $\mathrm{AB} 1 \# 3$ & $63-1000$ & $1 / 1024 b$ & $\mathrm{AB} 1 \# 6$ & $<1000$ & $1 / 1024 \mathrm{C}$ & AB1\#9 & $63-1000$ & $1 / 256 \mathrm{a}$ \\
\hline $\mathrm{AB} 1 \# 3$ & $63-1000$ & $1 / 1024 \mathrm{C}$ & $\mathrm{AB} 1 \# 6$ & $<1000$ & 1/16384B & $\mathrm{AB} 1 \# 9$ & $63-1000$ & $1 / 256 b$ \\
\hline $\mathrm{AB} 1 \# 3$ & $63-1000$ & $1 / 4096 \mathrm{~A}$ & $\mathrm{AB} 1 \# 6$ & $<1000$ & $1 / 16384 \mathrm{C}$ & $\mathrm{AB} 1 \# 9$ & $63-1000$ & $1 / 1024 \mathrm{~A}$ \\
\hline $\mathrm{AB} 1 \# 3$ & $63-1000$ & $1 / 4096 \mathrm{C}$ & AB1\#6 & $<1000$ & $1 / 16384 \mathrm{D}$ & $\mathrm{AB} 1 \# 9$ & $63-1000$ & $1 / 4096 \mathrm{~A}$ \\
\hline $\mathrm{AB} 1 \# 3$ & $63-1000$ & $1 / 4096 \mathrm{D}$ & AB1\#6 & $<63$ & $1 / 64$ & $\mathrm{AB} 1 \# 9$ & $63-1000$ & $1 / 4096 \mathrm{C}$ \\
\hline $\mathrm{AB} 1 \# 3$ & $<1000$ & $1 / 1024 \mathrm{~A}$ & AB1\#6 & $<63$ & $1 / 256 a$ & $\mathrm{AB} 1 \# 9$ & $63-1000$ & $1 / 4096 \mathrm{D}$ \\
\hline $\mathrm{AB} 1 \# 3$ & $<1000$ & $1 / 1024 \mathrm{~B}$ & AB1\#6 & $<63$ & $1 / 256 \mathrm{a}$ & AB1\#9 & $<1000$ & $1 / 1024 \mathrm{C}$ \\
\hline $\mathrm{AB} 1 \# 3$ & $<1000$ & $1 / 1024 \mathrm{C}$ & AB1\#6 & $<63$ & $1 / 256 b$ & AB1\#9 & $<1000$ & 1/4096B \\
\hline $\mathrm{AB} 1 \# 3$ & $<1000$ & 1/16384B & AB1\#6 & $<63$ & $1 / 1024$ & AB1\#9 & $<1000$ & 1/16384B \\
\hline $\mathrm{AB} 1 \# 3$ & $<1000$ & $1 / 16384 \mathrm{C}$ & AB1\#6 & $<63$ & $1 / 1024 \mathrm{~A}$ & AB1\#9 & $<1000$ & $1 / 16384 \mathrm{C}$ \\
\hline $\mathrm{AB} 1 \# 3$ & $<1000$ & $1 / 16384 \mathrm{D}$ & AB1\#6 & $<63$ & $1 / 1024 a$ & AB1\#9 & $<1000$ & $1 / 16384 \mathrm{D}$ \\
\hline $\mathrm{AB} 1 \# 3$ & $<63$ & $1 / 64(1)$ & AB1\#6 & $<63$ & $1 / 4096$ & AB1\#9 & $<63$ & $1 / 64$ \\
\hline $\mathrm{AB} 1 \# 3$ & $<63$ & $1 / 64(2)$ & AB1\#6 & $<63$ & $1 / 4096 \mathrm{~A}$ & AB1\#9 & $<63$ & $1 / 256 \mathrm{a}$ \\
\hline $\mathrm{AB} 1 \# 3$ & $<63$ & $1 / 256 a$ & $\mathrm{AB} 1 \# 7$ & $63-1000$ & $1 / 256 \mathrm{~A}$ & AB1\#9 & $<63$ & $1 / 1024$ \\
\hline $\mathrm{AB} 1 \# 3$ & $<63$ & $1 / 1024$ & $\mathrm{AB} 1 \# 7$ & $63-1000$ & $1 / 256 a$ & AB1\#9 & $<63$ & $1 / 1024 \mathrm{~A}$ \\
\hline $\mathrm{AB} 1 \# 3$ & $<63$ & $1 / 1024 \mathrm{~A}$ & $\mathrm{AB} 1 \# 7$ & $63-1000$ & $1 / 256 \mathrm{~B}$ & AB1\#9 & $<63$ & $1 / 4096$ \\
\hline $\mathrm{AB} 1 \# 3$ & $<63$ & $1 / 4096$ & $\mathrm{AB} 1 \# 7$ & $63-1000$ & $1 / 256 \mathrm{C}$ & $\mathrm{AB} 1 \# 9$ & $<63$ & $1 / 4096$ \\
\hline $\mathrm{AB} 1 \# 3$ & $<63$ & $1 / 4096$ & $\mathrm{AB} 1 \# 7$ & $63-1000$ & $1 / 1024 \mathrm{D}$ & $\mathrm{AB} 1 \# 9$ & $<63$ & $1 / 4096 \mathrm{~A}$ \\
\hline $\mathrm{AB} 1 \# 3$ & $<63$ & $1 / 4096 \mathrm{~A}$ & $\mathrm{AB} 1 \# 7$ & $63-1000$ & $1 / 4096 \mathrm{~A}$ & $\mathrm{AB} 1 \# 10$ & $63-1000$ & $1 / 256 \mathrm{a}$ \\
\hline $\mathrm{AB} 1 \# 4$ & $63-1000$ & $1 / 12288 \mathrm{D}$ & $\mathrm{AB} 1 \# 7$ & $63-1000$ & $1 / 4096 \mathrm{C}$ & $\mathrm{AB} 1 \# 10$ & $63-1000$ & $1 / 256 b$ \\
\hline $\mathrm{AB} 1 \# 4$ & $63<$ & $1 / 256 \mathrm{C}$ & $\mathrm{AB} 1 \# 7$ & $63-1000$ & $1 / 4096 \mathrm{D}$ & $\mathrm{AB} 1 \# 10$ & $63-1000$ & $1 / 1024 \mathrm{~A}$ \\
\hline $\mathrm{AB} 1 \# 4$ & $63<$ & $1 / 1024 \mathrm{C}$ & $\mathrm{AB} 1 \# 7$ & $<1000$ & $1 / 256 \mathrm{~A}$ & $\mathrm{AB} 1 \# 10$ & $63-1000$ & $1 / 4096 \mathrm{~A}$ \\
\hline $\mathrm{AB} 1 \# 4$ & $<1000$ & $1 / 4096 \mathrm{C}$ & $\mathrm{AB} 1 \# 7$ & $<1000$ & $1 / 256 \mathrm{~B}$ & $\mathrm{AB} 1 \# 10$ & $63-1000$ & $1 / 4096 \mathrm{C}$ \\
\hline $\mathrm{AB} 1 \# 4$ & $<1000$ & $1 / 12288 \mathrm{~A}$ & $\mathrm{AB} 1 \# 7$ & $<1000$ & $1 / 256 \mathrm{C}$ & $\mathrm{AB} 1 \# 10$ & $63-1000$ & $1 / 4096 \mathrm{D}$ \\
\hline $\mathrm{AB} 1 \# 4$ & $<1000$ & $1 / 12288 \mathrm{~B}$ & $\mathrm{AB} 1 \# 7$ & $<1000$ & 1/1024B & $\mathrm{AB} 1 \# 10$ & $<1000$ & $1 / 1024 \mathrm{~A}$ \\
\hline $\mathrm{AB} 1 \# 4$ & $<63$ & $1 / 64(1)$ & $\mathrm{AB} 1 \# 7$ & $<1000$ & $1 / 4096 \mathrm{C}$ & $\mathrm{AB} 1 \# 10$ & $<1000$ & $1 / 1024 \mathrm{C}$ \\
\hline $\mathrm{AB} 1 \# 4$ & $<63$ & $1 / 64(2)$ & $\mathrm{AB} 1 \# 7$ & $<1000$ & 1/16384B & $\mathrm{AB} 1 \# 10$ & $<1000$ & 1/16384B \\
\hline $\mathrm{AB} 1 \# 4$ & $<63$ & $1 / 1024 \mathrm{a}$ & $\mathrm{AB} 1 \# 7$ & $<1000$ & $1 / 16384 \mathrm{C}$ & $\mathrm{AB} 1 \# 10$ & $<1000$ & $1 / 16384 \mathrm{C}$ \\
\hline $\mathrm{AB} 1 \# 4$ & $<63$ & $1 / 12288 \mathrm{D}$ & $\mathrm{AB} 1 \# 7$ & $<1000$ & 1/16384D & $\mathrm{AB} 1 \# 10$ & $<1000$ & 1/16384D \\
\hline $\mathrm{AB} 1 \# 5$ & $63-1000$ & $1 / 256 \mathrm{a}$ & $\mathrm{AB} 1 \# 7$ & $<63$ & $1 / 64$ & $\mathrm{AB} 1 \# 10$ & $<63$ & $1 / 64$ \\
\hline $\mathrm{AB} 1 \# 5$ & $63-1000$ & $1 / 256 b$ & $\mathrm{AB} 1 \# 7$ & $<63$ & $1 / 64$ & $\mathrm{AB} 1 \# 10$ & $<63$ & $1 / 1024 \mathrm{~A}$ \\
\hline $\mathrm{AB} 1 \# 5$ & $63-1000$ & $1 / 1024$ & $\mathrm{AB} 1 \# 7$ & $<63$ & $1 / 256 a$ & $\mathrm{AB} 1 \# 10$ & $<63$ & $1 / 4096$ \\
\hline $\mathrm{AB} 1 \# 5$ & $63-1000$ & $1 / 1024 \mathrm{~A}$ & $\mathrm{AB} 1 \# 7$ & $<63$ & $1 / 256 b$ & $\mathrm{AB} 1 \# 10$ & $<63$ & $1 / 4096$ \\
\hline $\mathrm{AB} 1 \# 5$ & $63-1000$ & $1 / 1024 b$ & $\mathrm{AB} 1 \# 7$ & $<63$ & $1 / 1024$ & $\mathrm{AB} 1 \# 10$ & $<63$ & $1 / 4096 \mathrm{~A}$ \\
\hline $\mathrm{AB} 1 \# 5$ & $63-1000$ & $1 / 1024 \mathrm{C}$ & $\mathrm{AB} 1 \# 7$ & $<63$ & $1 / 1024 \mathrm{~A}$ & AB1\#11 & $63-1000$ & $1 / 256 b$ \\
\hline $\mathrm{AB} 1 \# 5$ & $63-1000$ & $1 / 4096 \mathrm{~A}$ & $\mathrm{AB} 1 \# 7$ & $<63$ & $1 / 1024 a$ & $\mathrm{AB} 1 \# 11$ & $63-1000$ & $1 / 1024 \mathrm{~A}$ \\
\hline $\mathrm{AB} 1 \# 5$ & $63-1000$ & $1 / 4096 \mathrm{C}$ & $\mathrm{AB} 1 \# 7$ & $<63$ & $1 / 4096$ & $\mathrm{AB} 1 \# 11$ & $63-1000$ & $1 / 4096 \mathrm{~A}$ \\
\hline $\mathrm{AB} 1 \# 5$ & $63-1000$ & $1 / 4096 \mathrm{D}$ & $\mathrm{AB} 1 \# 7$ & $<63$ & $1 / 4096$ & $\mathrm{AB} 1 \# 11$ & $63-1000$ & $1 / 4096 \mathrm{C}$ \\
\hline $\mathrm{AB} 1 \# 5$ & $<1000$ & $1 / 1024 \mathrm{~A}$ & $\mathrm{AB} 1 \# 7$ & $<63$ & $1 / 4096 \mathrm{~A}$ & AB1\#11 & $63-1000$ & $1 / 4096 \mathrm{D}$ \\
\hline $\mathrm{AB} 1 \# 5$ & $<1000$ & $1 / 1024 \mathrm{C}$ & $\mathrm{AB} 1 \# 8$ & $63-1000$ & $1 / 256 \mathrm{a}$ & $\mathrm{AB} 1 \# 11$ & $<1000$ & 1/4096B \\
\hline $\mathrm{AB} 1 \# 5$ & $<1000$ & 1/16384B & $\mathrm{AB} 1 \# 8$ & $63-1000$ & $1 / 256 b$ & $\mathrm{AB} 1 \# 11$ & $<1000$ & 1/16384B \\
\hline $\mathrm{AB} 1 \# 5$ & $<1000$ & $1 / 16384 C$ & $\mathrm{AB} 1 \# 8$ & $63-1000$ & $1 / 1024 \mathrm{~A}$ & $\mathrm{AB} 1 \# 11$ & $<1000$ & $1 / 16384 \mathrm{C}$ \\
\hline $\mathrm{AB} 1 \# 5$ & $<1000$ & $1 / 16384 \mathrm{D}$ & $\mathrm{AB} 1 \# 8$ & $63-1000$ & $1 / 4096 \mathrm{~A}$ & $\mathrm{AB} 1 \# 11$ & $<1000$ & $1 / 16384 \mathrm{D}$ \\
\hline $\mathrm{AB} 1 \# 5$ & $<63$ & $1 / 256 a$ & $\mathrm{AB} 1 \# 8$ & $63-1000$ & $1 / 4096 \mathrm{C}$ & $\mathrm{AB} 1 \# 11$ & $<63$ & $1 / 64$ \\
\hline $\mathrm{AB} 1 \# 5$ & $<63$ & $1 / 256 b$ & AB1\#8 & $63-1000$ & $1 / 4096 \mathrm{D}$ & AB1\#11 & $<63$ & $1 / 256 \mathrm{a}$ \\
\hline $\mathrm{AB} 1 \# 5$ & $<63$ & $1 / 1024$ & $\mathrm{AB} 1 \# 8$ & $<1000$ & $1 / 1024 \mathrm{~A}$ & AB1\#11 & $<63$ & $1 / 1024$ \\
\hline $\mathrm{AB} 1 \# 5$ & $<63$ & $1 / 1024 a$ & AB1\#8 & $<1000$ & $1 / 1024 \mathrm{C}$ & $\mathrm{AB} 1 \# 11$ & $<63$ & $1 / 1024 \mathrm{~A}$ \\
\hline $\mathrm{AB} 1 \# 5$ & $<63$ & $1 / 4096$ & AB1\#8 & $<1000$ & 1/16384B & AB1\#11 & $<63$ & $1 / 4096$ \\
\hline $\mathrm{AB} 1 \# 5$ & $<63$ & $1 / 4096$ & $\mathrm{AB} 1 \# 8$ & $<1000$ & $1 / 16384 \mathrm{C}$ & $\mathrm{AB} 1 \# 11$ & $<63$ & $1 / 4096$ \\
\hline $\mathrm{AB} 1 \# 5$ & $<63$ & $1 / 4096 \mathrm{~A}$ & $\mathrm{AB} 1 \# 8$ & $<1000$ & $1 / 16384 \mathrm{D}$ & AB1\#11 & $<63$ & $1 / 4096 \mathrm{~A}$ \\
\hline
\end{tabular}


Table 5 (Cont.)

\begin{tabular}{|c|c|c|c|c|c|c|c|c|}
\hline Sample ID & Size $(\mu \mathrm{m})$ & $\begin{array}{l}\text { Aliquot } \\
\text { size \& ID }\end{array}$ & Sample ID & Size $(\mu \mathrm{m})$ & $\begin{array}{l}\text { Aliquot } \\
\text { size \& ID }\end{array}$ & Sample ID & Size $(\mu \mathrm{m})$ & $\begin{array}{l}\text { Aliquot } \\
\text { size \& ID }\end{array}$ \\
\hline $\mathrm{AB} 1 \# 12$ & $<63$ & $1 / 64$ & $\mathrm{AB} 2 \# 8$ & $<1000$ & $1 / 256 \mathrm{~A}$ & AB3\#1 & $<63$ & $1 / 256 \mathrm{C}$ \\
\hline $\mathrm{AB} 1 \# 12$ & $<63$ & $1 / 256 \mathrm{~B}$ & $\mathrm{AB} 2 \# 8$ & $<1000$ & $1 / 256 \mathrm{~B}$ & AB3\#1 & $<63$ & $1 / 1024 \mathrm{~A}$ \\
\hline $\mathrm{AB} 1 \# 12$ & $<63$ & $1 / 256 \mathrm{C}$ & $\mathrm{AB} 2 \# 8$ & $<1000$ & 1/1024B & AB3\#1 & $<63$ & 1/1024B \\
\hline $\mathrm{AB} 1 \# 13$ & $<1000$ & $1 / 1024$ & $\mathrm{AB} 2 \# 8$ & $<1000$ & $1 / 1024 \mathrm{C}$ & AB3\#1 & $<63$ & $1 / 4096 \mathrm{~B}(3)$ \\
\hline AB2\#1 & $<1000$ & $1 / 256 \mathrm{~A}$ & $\mathrm{AB} 2 \# 8$ & $<63$ & $1 / 64$ & AB3\#1 & $<63$ & $1 / 4096 \mathrm{C}$ \\
\hline $\mathrm{AB} 2 \# 1$ & $<1000$ & $1 / 256 \mathrm{~B}$ & $\mathrm{AB} 2 \# 8$ & $<63$ & $1 / 256 \mathrm{C}$ & AB3\#1 & $<63$ & $1 / 12288 \mathrm{~A}$ \\
\hline AB2\#1 & $<1000$ & 1/1024B & $\mathrm{AB} 2 \# 9$ & $63-1000$ & $1 / 256 \mathrm{D}$ & AB3\#1 & $<63$ & $1 / 12288 \mathrm{D}$ \\
\hline AB2\#1 & $<1000$ & $1 / 1024 \mathrm{C}$ & $\mathrm{AB} 2 \# 9$ & $63-1000$ & $1 / 12288 \mathrm{D}$ & AB3\#1 & $<63$ & $1 / 12288 \mathrm{D}$ \\
\hline $\mathrm{AB} 2 \# 1$ & $<63$ & $1 / 64(1)$ & $\mathrm{AB} 2 \# 9$ & $<1000$ & $1 / 1024$ & $\mathrm{AB} 3 \# 2$ & $<1000$ & $1 / 1024 \mathrm{~A}$ \\
\hline $\mathrm{AB} 2 \# 1$ & $<63$ & $1 / 64(2)$ & $\mathrm{AB} 2 \# 9$ & $<1000$ & 1/1024B & AB3\#2 & $<1000$ & 1/1024B \\
\hline $\mathrm{AB} 2 \# 1$ & $<63$ & $1 / 256 \mathrm{C}$ & $\mathrm{AB} 2 \# 9$ & $<1000$ & $1 / 1024 \mathrm{C}$ & AB3\#2 & $<1000$ & $1 / 1024 \mathrm{C}$ \\
\hline AB2\#2 & $<1000$ & $1 / 256 \mathrm{~A}$ & $\mathrm{AB} 2 \# 9$ & $<1000$ & $1 / 12288$ & AB3\#2 & $<1000$ & $1 / 4096 \mathrm{C}$ \\
\hline $\mathrm{AB} 2 \# 2$ & $<1000$ & $1 / 256 \mathrm{~B}$ & $\mathrm{AB} 2 \# 9$ & $<1000$ & $1 / 12288 \mathrm{~A}$ & AB3\#2 & $<1000$ & 1/16384B \\
\hline $\mathrm{AB} 2 \# 2$ & $<1000$ & 1/1024B & $\mathrm{AB} 2 \# 9$ & $<63$ & $1 / 256 \mathrm{D}$ & AB3\#2 & $<1000$ & $1 / 16384 \mathrm{C}$ \\
\hline $\mathrm{AB} 2 \# 2$ & $<1000$ & $1 / 1024 \mathrm{C}$ & $\mathrm{AB} 2 \# 9$ & $<63$ & $2 / 1024$ & $\mathrm{AB} 3 \# 2$ & $<1000$ & $1 / 16384 \mathrm{D}$ \\
\hline $\mathrm{AB} 2 \# 2$ & $<1000$ & $1 / 4096 \mathrm{~A}$ & $\mathrm{AB} 2 \# 9$ & $<63$ & $1 / 1024$ & $\mathrm{AB} 3 \# 2$ & $<63$ & $1 / 64$ \\
\hline $\mathrm{AB} 2 \# 2$ & $<1000$ & 1/4096B & $\mathrm{AB} 2 \# 9$ & $<63$ & $1 / 12288 \mathrm{D}$ & AB3\#2 & $<63$ & $1 / 256 \mathrm{~B}$ \\
\hline $\mathrm{AB} 2 \# 2$ & $<63$ & $1 / 64$ & AB2\#10 & 63-1000 & $1 / 4096 \mathrm{~A}$ & AB3\#2 & $<63$ & $1 / 256 \mathrm{C}$ \\
\hline AB2\#2 & $<63$ & $1 / 256 \mathrm{C}$ & AB2\#10 & $63-1000$ & $1 / 12288 d$ & AB3\#2 & $<63$ & $1 / 1024 \mathrm{~A}$ \\
\hline $\mathrm{AB} 2 \# 3$ & $<1000$ & $1 / 256 \mathrm{~A}$ & AB2\#10 & $<1000$ & $1 / 256 \mathrm{~A}$ & AB3\#2 & $<63$ & 1/1024B \\
\hline $\mathrm{AB} 2 \# 3$ & $<1000$ & $1 / 256 \mathrm{~B}$ & AB2\#10 & $<1000$ & $1 / 12288 \mathrm{~A}$ & AB3\#2 & $<63$ & 1/4096B \\
\hline $\mathrm{AB} 2 \# 3$ & $<1000$ & 1/1024B & AB2\#10 & $<1000$ & 1/12288B & AB3\#2 & $<63$ & $1 / 4096 \mathrm{C}$ \\
\hline $\mathrm{AB} 2 \# 3$ & $<1000$ & $1 / 1024 \mathrm{C}$ & $\mathrm{AB} 2 \# 10$ & $<63$ & $1 / 256 \mathrm{D}$ & $\mathrm{AB} 3 \# 2$ & $<63$ & $1 / 12288 \mathrm{~A}$ \\
\hline $\mathrm{AB} 2 \# 3$ & $<1000$ & $1 / 4096 \mathrm{~A}$ & $\mathrm{AB} 2 \# 10$ & $<63$ & $1 / 1024 d$ & $\mathrm{AB} 3 \# 2$ & $<63$ & $1 / 12288 \mathrm{C}$ \\
\hline $\mathrm{AB} 2 \# 3$ & $<1000$ & $1 / 4096 \mathrm{~B}$ & AB2\#10 & $<63$ & $1 / 12288 d$ & AB3\#2 & $<63$ & $1 / 12288 \mathrm{D}$ \\
\hline $\mathrm{AB} 2 \# 3$ & $<63$ & $1 / 64$ & AB2\#11 & 63-1000 & 1/4096A & $\mathrm{AB} 3 \# 3$ & 63-1000 & $1 / 12288 \mathrm{D}$ \\
\hline $\mathrm{AB} 2 \# 3$ & $<63$ & $1 / 256 \mathrm{C}$ & AB2\#11 & 63-1000 & $1 / 12288 d$ & AB3\#3 & $<1000$ & $1 / 4096 \mathrm{C}$ \\
\hline $\mathrm{AB} 2 \# 4$ & $<1000$ & $1 / 256 \mathrm{~A}$ & AB2\#11 & $<1000$ & $1 / 256 \mathrm{~A}$ & $\mathrm{AB} 3 \# 3$ & $<1000$ & $1 / 12288 \mathrm{~A}$ \\
\hline $\mathrm{AB} 2 \# 4$ & $<1000$ & $1 / 256 \mathrm{~B}$ & AB2\#11 & $<1000$ & $1 / 1024 \mathrm{C}$ & $\mathrm{AB} 3 \# 3$ & $<1000$ & 1/12288B \\
\hline $\mathrm{AB} 2 \# 4$ & $<1000$ & 1/1024B & AB2\#11 & $<1000$ & $1 / 12288 \mathrm{~A}$ & $\mathrm{AB} 3 \# 3$ & $<63$ & $1 / 64$ \\
\hline $\mathrm{AB} 2 \# 4$ & $<1000$ & $1 / 1024 \mathrm{C}$ & AB2\#11 & $<1000$ & 1/12288B & $\mathrm{AB} 3 \# 3$ & $<63$ & $1 / 128$ \\
\hline $\mathrm{AB} 2 \# 4$ & $<1000$ & 1/4096B & AB2\#11 & $<63$ & $1 / 256 \mathrm{D}$ & $\mathrm{AB} 3 \# 3$ & $<63$ & $1 / 1024 \mathrm{~A}$ \\
\hline $\mathrm{AB} 2 \# 4$ & $<1000$ & $1 / 4096 \mathrm{C}$ & AB2\#11 & $<63$ & $1 / 4096 \mathrm{~A}$ & AB3\#3 & $<63$ & $1 / 12288 \mathrm{D}$ \\
\hline $\mathrm{AB} 2 \# 4$ & $<63$ & $1 / 64(1)$ & AB2\#11 & $<63$ & $1 / 12288 \mathrm{~d}$ & AB3\#4 & 63-1000 & $1 / 12288 \mathrm{D}$ \\
\hline AB2\#4 & $<63$ & $1 / 64(2)$ & $\mathrm{AB} 2 \# 12$ & $63-1000$ & 1/4096A & $\mathrm{AB} 3 \# 4$ & 63-1000 & $1 / 12288 \mathrm{~d}$ \\
\hline $\mathrm{AB} 2 \# 4$ & $<63$ & $1 / 256 \mathrm{C}$ & AB2\#12 & $63-1000$ & $1 / 12288 d$ & $\mathrm{AB} 3 \# 4$ & $<1000$ & 1/1024B \\
\hline $\mathrm{AB} 2 \# 5$ & $<1000$ & $1 / 256 \mathrm{~A}$ & $\mathrm{AB} 2 \# 12$ & $<1000$ & $1 / 256 \mathrm{~A}$ & $\mathrm{AB} 3 \# 4$ & $<1000$ & $1 / 1024 \mathrm{C}$ \\
\hline $\mathrm{AB} 2 \# 5$ & $<1000$ & $1 / 256 \mathrm{~B}$ & $\mathrm{AB} 2 \# 12$ & $<1000$ & $1 / 256 \mathrm{~B}$ & $\mathrm{AB} 3 \# 4$ & $<1000$ & $1 / 1024 \mathrm{C}$ \\
\hline $\mathrm{AB} 2 \# 5$ & $<1000$ & 1/1024B & AB2\#12 & $<1000$ & 1/1024B & AB3\#4 & $<1000$ & $1 / 4096 \mathrm{~B}$ \\
\hline $\mathrm{AB} 2 \# 5$ & $<1000$ & $1 / 1024 \mathrm{C}$ & $\mathrm{AB} 2 \# 12$ & $<1000$ & $1 / 12288 \mathrm{~A}$ & AB3\#4 & $<1000$ & $1 / 4096 \mathrm{C}$ \\
\hline $\mathrm{AB} 2 \# 5$ & $<1000$ & $1 / 4096 \mathrm{~A}$ & AB2\#12 & $<1000$ & 1/12288B & AB3\#4 & $<1000$ & 1/4096C \\
\hline $\mathrm{AB} 2 \# 5$ & $<63$ & $1 / 64$ & $\mathrm{AB} 2 \# 12$ & $<63$ & $1 / 64$ & $\mathrm{AB} 3 \# 4$ & $<1000$ & $1 / 12288 \mathrm{~A}$ \\
\hline $\mathrm{AB} 2 \# 5$ & $<63$ & $1 / 256 \mathrm{C}$ & AB2\#12 & $<63$ & $1 / 256 \mathrm{D}$ & AB3\#4 & $<1000$ & $1 / 12288 \mathrm{~A}$ \\
\hline $\mathrm{AB} 2 \# 6$ & $<1000$ & $1 / 256 \mathrm{~A}$ & AB2\#12 & $<63$ & $1 / 4096 \mathrm{~A}$ & AB3\#4 & $<1000$ & $1 / 12288 \mathrm{~A}$ \\
\hline $\mathrm{AB} 2 \# 6$ & $<1000$ & $1 / 256 \mathrm{~B}$ & AB2\#12 & $<63$ & $1 / 12288 \mathrm{~d}$ & $\mathrm{AB} 3 \# 4$ & $<1000$ & 1/12288B \\
\hline $\mathrm{AB} 2 \# 6$ & $<1000$ & $1 / 1024 \mathrm{C}$ & AB2\#13 & $<63$ & $1 / 64$ & $\mathrm{AB} 3 \# 4$ & $<1000$ & $1 / 12288 \mathrm{C}$ \\
\hline $\mathrm{AB} 2 \# 6$ & $<1000$ & $1 / 4096 \mathrm{~A}$ & AB2\#13 & $<63$ & $1 / 256 \mathrm{C}$ & AB3\#4 & $<1000$ & $1 / 12288 \mathrm{C}$ \\
\hline $\mathrm{AB} 2 \# 6$ & $<1000$ & $1 / 4096 \mathrm{C}$ & AB2\#13 & $<63$ & $1 / 256 \mathrm{D}$ & AB3\#4 & $<63$ & $1 / 64$ \\
\hline $\mathrm{AB} 2 \# 6$ & $<63$ & $1 / 64$ & AB3\#1 & $<1000$ & $1 / 1024 \mathrm{~A}$ & $\mathrm{AB} 3 \# 4$ & $<63$ & $1 / 256 \mathrm{C}$ \\
\hline $\mathrm{AB} 2 \# 6$ & $<63$ & $1 / 256 \mathrm{D}$ & AB3\#1 & $<1000$ & 1/1024B & AB3\#4 & $<63$ & $1 / 12288 d$ \\
\hline $\mathrm{AB} 2 \# 7$ & $<1000$ & $1 / 256 \mathrm{~A}$ & AB3\#1 & $<1000$ & $1 / 1024 \mathrm{C}$ & $\mathrm{AB} 3 \# 4$ & $<63$ & 1/12288D \\
\hline $\mathrm{AB} 2 \# 7$ & $<1000$ & 1/1024B & AB3\#1 & $<1000$ & 1/4096B & $\mathrm{AB} 3 \# 5$ & $63-1000$ & 1/12288D \\
\hline $\mathrm{AB} 2 \# 7$ & $<1000$ & $1 / 4096 \mathrm{~A}$ & AB3\#1 & $<1000$ & $1 / 16384 \mathrm{C}$ & AB3\#5 & $<1000$ & $1 / 1024 \mathrm{C}$ \\
\hline $\mathrm{AB} 2 \# 7$ & $<1000$ & $1 / 4096 \mathrm{C}$ & AB3\#1 & $<1000$ & 1/16384D & $\mathrm{AB} 3 \# 5$ & $<1000$ & $1 / 4096 \mathrm{C}$ \\
\hline $\mathrm{AB} 2 \# 7$ & $<63$ & $1 / 64$ & $\mathrm{AB} 3 \# 1$ & $<1000$ & 1/16384D & $\mathrm{AB} 3 \# 5$ & $<1000$ & $1 / 12288 \mathrm{~A}$ \\
\hline $\mathrm{AB} 2 \# 7$ & $<63$ & $1 / 256 \mathrm{~B}$ & AB3\#1 & $<63$ & $1 / 64$ & $\mathrm{AB} 3 \# 5$ & $<1000$ & $1 / 12288 \mathrm{C}$ \\
\hline $\mathrm{AB} 2 \# 7$ & $<63$ & $1 / 256 \mathrm{C}$ & AB3\#1 & $<63$ & $1 / 256 \mathrm{~B}$ & $\mathrm{AB} 3 \# 5$ & $<63$ & $1 / 64$ \\
\hline
\end{tabular}


Kozo Takahashi, Hirofumi Asahi, Yusuke Okazaki, Jonaotaro Onodera, Hideto Tsutsui,

Takahito Ikenoue, Yoshiyuki Kanematsu, Seiji Tanaka and Shinya Iwasaki

Table 5 (Cont.)

\begin{tabular}{|c|c|c|c|c|c|c|c|c|}
\hline Sample ID & Size $(\mu \mathrm{m})$ & $\begin{array}{l}\text { Aliquot } \\
\text { size \& ID }\end{array}$ & Sample ID & Size $(\mu \mathrm{m})$ & $\begin{array}{l}\text { Aliquot } \\
\text { size \& ID }\end{array}$ & Sample ID & Size $(\mu \mathrm{m})$ & $\begin{array}{l}\text { Aliquot } \\
\text { size \& ID }\end{array}$ \\
\hline $\mathrm{AB} 3 \# 5$ & $<63$ & $1 / 256 \mathrm{C}$ & AB3\#10 & $<63$ & $1 / 12288 d$ & $\overline{\mathrm{AB} 4 \# 8}$ & $<63$ & $1 / 64$ \\
\hline $\mathrm{AB} 3 \# 5$ & $<63$ & $1 / 12288 \mathrm{D}$ & AB3\#10 & $<63$ & $1 / 12288 \mathrm{D}$ & AB4\#8 & $<63$ & $1 / 256 \mathrm{C}$ \\
\hline AB3\#6 & $63-1000$ & $1 / 12288 \mathrm{D}$ & AB3\#11 & $63-1000$ & $1 / 12288 \mathrm{D}$ & AB4\#9 & $<1000$ & $1 / 256 \mathrm{~B}$ \\
\hline AB3\#6 & $<1000$ & 1/1024B & AB3\#11 & $<1000$ & 1/4096B & AB4\#9 & $<1000$ & $1 / 4096 \mathrm{~d}(7)$ \\
\hline AB3\#6 & $<1000$ & $1 / 4096 \mathrm{C}$ & AB3\#11 & $<1000$ & $1 / 12288 \mathrm{~A}$ & AB4\#9 & $<63$ & $1 / 64$ \\
\hline AB3\#6 & $<1000$ & $1 / 12288 \mathrm{~A}$ & AB3\#11 & $<1000$ & 1/12288B & AB4\#9 & $<63$ & $1 / 256 \mathrm{C}$ \\
\hline $\mathrm{AB} 3 \# 6$ & $<1000$ & $1 / 12288 \mathrm{~B}$ & AB3\#11 & $<63$ & $1 / 64$ & $\mathrm{AB} 4 \# 10$ & $<1000$ & $1 / 256 \mathrm{~B}$ \\
\hline AB3\#6 & $<63$ & $1 / 64$ & AB3\#11 & $<63$ & $1 / 256$ & AB4\#10 & $<1000$ & $1 / 1024 \mathrm{~A}$ \\
\hline AB3\#6 & $<63$ & $1 / 256 \mathrm{C}$ & AB3\#11 & $<63$ & $1 / 256 \mathrm{C}$ & AB4\#10 & $<1000$ & $1 / 1024 \mathrm{~B}$ \\
\hline AB3\#6 & $<63$ & $1 / 12288 \mathrm{D}$ & AB3\#11 & $<63$ & $1 / 12288 \mathrm{D}$ & AB4\#10 & $<1000$ & $1 / 1024 \mathrm{~B}$ \\
\hline $\mathrm{AB} 3 \# 7$ & $63-1000$ & $1 / 12288 \mathrm{D}$ & AB4\#1 & $<1000$ & $1 / 256 \mathrm{~B}$ & $\mathrm{AB} 4 \# 10$ & $<1000$ & 1/4096A \\
\hline $\mathrm{AB} 3 \# 7$ & $<1000$ & $1 / 64$ & AB4\#1 & $<1000$ & $1 / 4096 \mathrm{D}$ & AB4\#10 & $<1000$ & $1 / 4096 \mathrm{~B}$ \\
\hline $\mathrm{AB} 3 \# 7$ & $<1000$ & 1/1024B & AB4\#1 & $<63$ & $1 / 64$ & AB4\#10 & $<1000$ & $1 / 4096 \mathrm{C}$ \\
\hline AB3\#7 & $<1000$ & $1 / 4096 \mathrm{~B}$ & AB4\#1 & $<63$ & $1 / 256 \mathrm{C}$ & AB4\#10 & $<1000$ & $1 / 12288 \mathrm{~B}$ \\
\hline $\mathrm{AB} 3 \# 7$ & $<1000$ & $1 / 4096 \mathrm{C}$ & $\mathrm{AB} 4 \# 2$ & $<1000$ & $1 / 256 \mathrm{~B}$ & AB4\#10 & $<1000$ & $1 / 16384 \mathrm{~A}$ \\
\hline AB3\#7 & $<1000$ & $1 / 4096 \mathrm{C}$ & $\mathrm{AB} 4 \# 2$ & $<1000$ & $1 / 4096 \mathrm{D}(6)$ & AB4\#10 & $<1000$ & $1 / 16384 \mathrm{D}$ \\
\hline $\mathrm{AB} 3 \# 7$ & $<1000$ & $1 / 12288 \mathrm{~A}$ & AB4\#2 & $<63$ & $1 / 64(1)$ & AB4\#10 & $<63$ & $1 / 64$ \\
\hline $\mathrm{AB} 3 \# 7$ & $<1000$ & $1 / 12288 \mathrm{~A}$ & $\mathrm{AB} 4 \# 2$ & $<63$ & $1 / 64(2)$ & AB4\#10 & $<63$ & $1 / 256 \mathrm{C}$ \\
\hline AB3\#7 & $<1000$ & $1 / 12288 \mathrm{~B}$ & $\mathrm{AB} 4 \# 2$ & $<63$ & $1 / 256 \mathrm{C}$ & AB4\#11 & $<1000$ & $1 / 256 \mathrm{~B}$ \\
\hline AB3\#7 & $<1000$ & $1 / 12288 \mathrm{~B}$ & AB4\#3 & $<1000$ & $1 / 256 \mathrm{~B}$ & AB4\#11 & $<1000$ & $1 / 1024 \mathrm{~A}$ \\
\hline AB3\#7 & $<1000$ & $1 / 12288 \mathrm{C}$ & AB4\#3 & $<1000$ & $1 / 4096 \mathrm{D}$ & AB4\#11 & $<1000$ & $1 / 1024 \mathrm{~B}$ \\
\hline AB3\#7 & $<1000$ & $1 / 12288 \mathrm{C}$ & AB4\#3 & $<63$ & $1 / 256 \mathrm{C}$ & AB4\#11 & $<1000$ & $1 / 1024 \mathrm{C}$ \\
\hline $\mathrm{AB} 3 \# 7$ & $<63$ & $1 / 256 \mathrm{C}$ & $\mathrm{AB} 4 \# 4$ & $<1000$ & $1 / 256 \mathrm{~B}$ & AB4\#11 & $<1000$ & $1 / 1024 \mathrm{C}$ \\
\hline AB3\#7 & $<63$ & $1 / 12288 \mathrm{D}$ & AB4\#4 & $<1000$ & $1 / 4096 \mathrm{C} \mathrm{A}-2$ & AB4\#11 & $<1000$ & $1 / 4096 \mathrm{~A}$ \\
\hline AB3\#8 & $63-1000$ & $1 / 12288 \mathrm{D}$ & $\mathrm{AB} 4 \# 4$ & $<63$ & $1 / 64$ & AB4\#11 & $<1000$ & $1 / 4096 \mathrm{C}$ \\
\hline AB3\#8 & $<1000$ & 1/4096B & $\mathrm{AB} 4 \# 4$ & $<63$ & $1 / 256 \mathrm{C}$ & AB4\#11 & $<1000$ & $1 / 12288 \mathrm{~B}$ \\
\hline AB3\#8 & $<1000$ & $1 / 12288 \mathrm{~A}$ & AB4\#5 & $63-1000$ & SEM & $\mathrm{AB} 4 \# 11$ & $<1000$ & $1 / 16384 \mathrm{~A}$ \\
\hline $\mathrm{AB} 3 \# 8$ & $<1000$ & $1 / 12288 \mathrm{C}$ & AB4\#5 & $<1000$ & 1/1024B & AB4\#11 & $<1000$ & 1/16384D \\
\hline AB3\#8 & $<63$ & $1 / 64$ & AB4\#5 & $<63$ & $1 / 64$ & $\mathrm{AB} 4 \# 11$ & $<63$ & $1 / 64$ \\
\hline $\mathrm{AB} 3 \# 8$ & $<63$ & $1 / 128$ & $\mathrm{AB} 4 \# 5$ & $<63$ & $1 / 256 \mathrm{~B}$ & AB4\#11 & $<63$ & $1 / 256 \mathrm{C}$ \\
\hline AB3\#8 & $<63$ & $1 / 256 \mathrm{C}$ & AB4\#5 & $<63$ & $1 / 256 \mathrm{C}$ & $\mathrm{AB} 4 \# 12$ & $<1000$ & $1 / 256 \mathrm{~B}$ \\
\hline AB3\#8 & $<63$ & $1 / 12288 \mathrm{D}$ & AB4\#5 & & 1/1024A' & AB4\#12 & $<1000$ & $1 / 256 \mathrm{C}$ \\
\hline AB3\#9 & $63-1000$ & $1 / 12288 \mathrm{D}$ & AB4\#5 & & 1/1024B' & $\mathrm{AB} 4 \# 12$ & $<1000$ & $1 / 1024 \mathrm{~A}$ \\
\hline AB3\#9 & $<1000$ & 1/1024B & $\mathrm{AB} 4 \# 5$ & & $1 / 1024 C^{\prime}$ & AB4\#12 & $<1000$ & $1 / 1024 \mathrm{C}$ \\
\hline AB3\#9 & $<1000$ & 1/12288B & $\mathrm{AB} 4 \# 5$ & & $1 / 4096 A^{\prime}$ & AB4\#12 & $<1000$ & $1 / 4096 \mathrm{~A}$ \\
\hline AB3\#9 & $<1000$ & $1 / 12288 \mathrm{C}$ & AB4\#5 & & 1/4096B' & AB4\#12 & $<1000$ & $1 / 4096 \mathrm{D} \mathrm{A}-3$ \\
\hline AB3\#9 & $<63$ & $1 / 64$ & AB4\#5 & & 1/4096D' & AB5\#1 & $<1000$ & $1 / 1024 \mathrm{~A}$ \\
\hline AB3\#9 & $<63$ & $1 / 128$ & AB4\#6 & $<1000$ & $1 / 256 \mathrm{~B}$ & AB5\#1 & $<1000$ & 1/1024B \\
\hline AB3\#9 & $<63$ & $1 / 256 \mathrm{C}$ & AB4\#6 & $<1000$ & $1 / 1024 \mathrm{C}$ & AB5\#1 & $<1000$ & $1 / 16384 \mathrm{~A}$ \\
\hline AB3\#9 & $<63$ & $1 / 12288 \mathrm{D}$ & AB4\#6 & $<63$ & $1 / 64$ & AB5\#1 & $<1000$ & 1/16384B \\
\hline AB3\#10 & $63-1000$ & $1 / 12288 \mathrm{D}$ & AB4\#6 & $<63$ & $1 / 256 \mathrm{C}$ & AB5\#1 & $<1000$ & $1 / 16384 \mathrm{D}$ \\
\hline AB3\#10 & $63-1000$ & $1 / 12288 d$ & AB4\#6 & & $1 / 1024 A^{\prime}$ & AB5\#1 & $<63$ & $3 / 1024$ \\
\hline AB3\#10 & $<1000$ & $1 / 1024 \mathrm{~B}$ & AB4\#6 & & 1/1024B' & $\mathrm{AB} 5 \# 2$ & $<1000$ & $1 / 1024 \mathrm{~A}$ \\
\hline AB3\#10 & $<1000$ & $1 / 1024 \mathrm{~B}$ & AB4\#6 & & $1 / 1024 C^{\prime}$ & $\mathrm{AB} 5 \# 2$ & $<1000$ & 1/16384B \\
\hline AB3\#10 & $<1000$ & $1 / 1024 \mathrm{C}$ & AB4\#6 & & 1/4096A' & AB5\#2 & $<1000$ & $1 / 16384 \mathrm{D}$ \\
\hline AB3\#10 & $<1000$ & $1 / 1024 \mathrm{C}$ & AB4\#6 & & 1/4096C' & $\mathrm{AB} 5 \# 2$ & $<63$ & $3 / 1024$ \\
\hline $\mathrm{AB} 3 \# 10$ & $<1000$ & $1 / 4096 \mathrm{~B}$ & AB4\#6 & & 1/4096D' & AB5\#3 & $<1000$ & $1 / 1024 \mathrm{~B}$ \\
\hline $\mathrm{AB} 3 \# 10$ & $<1000$ & $1 / 4096 \mathrm{C}$ & AB4\#7 & $<1000$ & $1 / 256 \mathrm{~B}$ & AB5\#3 & $<1000$ & $1 / 1024 \mathrm{C}$ \\
\hline AB3\#10 & $<1000$ & $1 / 4096 \mathrm{C}$ & AB4\#7 & $<1000$ & $1 / 1024 \mathrm{~B}$ & AB5\#3 & $<1000$ & 1/4096B \\
\hline $\mathrm{AB} 3 \# 10$ & $<1000$ & $1 / 12288 \mathrm{~A}$ & AB4\#7 & $<1000$ & $1 / 4096 \mathrm{C}$ & AB5\#3 & $<1000$ & $1 / 16384 \mathrm{~A}$ \\
\hline AB3\#10 & $<1000$ & $1 / 12288 \mathrm{~A}$ & AB4\#7 & $<63$ & $1 / 64$ & AB5\#3 & $<1000$ & 1/16384B \\
\hline AB3\#10 & $<1000$ & 1/12288B & AB4\#7 & $<63$ & $1 / 256 \mathrm{C}$ & AB5\#3 & $<1000$ & $1 / 16384 \mathrm{D}$ \\
\hline AB3\#10 & $<1000$ & $1 / 12288 \mathrm{~B}$ & AB4\#8 & $<1000$ & $1 / 256 \mathrm{~B}$ & AB5\#3 & $<63$ & $3 / 1024$ \\
\hline AB3\#10 & $<1000$ & $1 / 12288 \mathrm{C}$ & AB4\#8 & $<1000$ & $1 / 4096 \mathrm{~A}$ & $\mathrm{AB} 5 \# 4$ & $<1000$ & $1 / 1024 \mathrm{~A}$ \\
\hline AB3\#10 & $<63$ & $1 / 64$ & AB4\#8 & $<1000$ & 1/12288B A-1 & AB5\#4 & $<1000$ & $1 / 1024 \mathrm{~B}$ \\
\hline $\mathrm{AB} 3 \# 10$ & $<63$ & $1 / 256 \mathrm{C}$ & AB4\#8 & $<1000$ & $1 / 16384 \mathrm{~A}$ & AB5\#4 & $<1000$ & 1/4096B \\
\hline AB3\#10 & $<63$ & 1/4096a & AB4\#8 & $<1000$ & $1 / 16384 \mathrm{D}$ & $\mathrm{AB} 5 \# 4$ & $<1000$ & $1 / 16384 \mathrm{~A}$ \\
\hline
\end{tabular}


Table 5 (Cont.)

\begin{tabular}{|c|c|c|c|c|c|c|c|c|}
\hline Sample ID & Size $(\mu \mathrm{m})$ & $\begin{array}{l}\text { Aliquot } \\
\text { size \& ID }\end{array}$ & Sample ID & Size $(\mu \mathrm{m})$ & $\begin{array}{l}\text { Aliquot } \\
\text { size \& ID }\end{array}$ & Sample ID & Size $(\mu \mathrm{m})$ & $\begin{array}{l}\text { Aliquot } \\
\text { size \& ID }\end{array}$ \\
\hline AB5\#4 & $<1000$ & 1/16384B & $\overline{\mathrm{AB} 6 \# 1}$ & $<1000$ & 1/16384B & AB6\#9 & $<1000$ & $1 / 16384 \mathrm{C}$ \\
\hline AB5\#4 & $<1000$ & 1/16384D & AB6\#1 & $<1000$ & $1 / 16384 \mathrm{C}$ & AB6\#9 & $<1000$ & 1/16384D \\
\hline AB5\#4 & $<63$ & $3 / 1024$ & AB6\#1 & $<1000$ & $1 / 16384 \mathrm{D}$ & AB6\#10 & $63-1000$ & $1 / 4096 \mathrm{~B}$ \\
\hline $\mathrm{AB} 5 \# 5$ & $<1000$ & 1/1024B & AB6\#1 & $<63$ & $1 / 4096 \mathrm{~A}$ & AB6\#10 & $<1000$ & $1 / 1024 \mathrm{~A}$ \\
\hline AB5\#5 & $<1000$ & 1/16384B & AB6\#2 & 63-1000 & 1/4096B & AB6\#10 & $<1000$ & $1 / 4096 \mathrm{C}$ \\
\hline AB5\#5 & $<1000$ & 1/16384D & AB6\#2 & $<1000$ & $1 / 1024 \mathrm{~A}$ & AB6\#10 & $<1000$ & 1/16384B \\
\hline AB5\#5 & $<63$ & $3 / 1024$ & AB6\#2 & $<1000$ & $1 / 4096 \mathrm{C}$ & AB6\#10 & $<1000$ & $1 / 16384 \mathrm{C}$ \\
\hline AB5\#6 & $<1000$ & $1 / 1024 \mathrm{~A}$ & AB6\#2 & $<1000$ & 1/16384B & AB6\#10 & $<1000$ & 1/16384D \\
\hline AB5\#6 & $<1000$ & 1/1024B & AB6\#2 & $<1000$ & $1 / 16384 \mathrm{C}$ & AB6\#11 & $63-1000$ & 1/4096B \\
\hline AB5\#6 & $<1000$ & $1 / 1024 \mathrm{C}$ & AB6\#2 & $<1000$ & 1/16384D & AB6\#11 & $<1000$ & $1 / 1024 \mathrm{~A}$ \\
\hline AB5\#6 & $<1000$ & $1 / 16384 \mathrm{~A}$ & AB6\#2 & $<63$ & 1/4096B & AB6\#11 & $<1000$ & $1 / 4096 \mathrm{C}$ \\
\hline AB5\#6 & $<1000$ & 1/16384B & AB6\#3 & $63-1000$ & 1/4096B & AB6\#11 & $<1000$ & 1/16384B \\
\hline AB5\#6 & $<1000$ & 1/16384D & AB6\#3 & $<1000$ & $1 / 1024 \mathrm{~A}$ & AB6\#11 & $<1000$ & $1 / 16384 \mathrm{C}$ \\
\hline AB5\#6 & $<63$ & $3 / 1024$ & AB6\#3 & $<1000$ & $1 / 4096 \mathrm{C}$ & AB6\#11 & $<1000$ & 1/16384D \\
\hline AB5\#7 & $<1000$ & $1 / 1024 \mathrm{~A}$ & AB6\#3 & $<1000$ & 1/16384B & AB6\#11 & $<63$ & $1 / 4096 \mathrm{~B}$ \\
\hline AB5\#7 & $<1000$ & 1/1024B & AB6\#3 & $<1000$ & $1 / 16384 \mathrm{C}$ & AB6\#12 & $63-1000$ & 1/4096B \\
\hline AB5\#7 & $<1000$ & $1 / 1024 \mathrm{C}$ & AB6\#3 & $<1000$ & 1/16384D & AB6\#12 & $<1000$ & $1 / 1024 \mathrm{~A}$ \\
\hline AB5\#7 & $<1000$ & $1 / 16384 \mathrm{~A}$ & AB6\#3 & $<63$ & 1/4096B & AB6\#12 & $<1000$ & $1 / 4096 \mathrm{C}$ \\
\hline AB5\#7 & $<1000$ & 1/16384B & AB6\#4 & $63-1000$ & $1 / 4096 \mathrm{~B}$ & AB6\#12 & $<1000$ & 1/16384B \\
\hline AB5\#7 & $<1000$ & 1/16384D & AB6\#4 & $<1000$ & $1 / 1024 \mathrm{~A}$ & AB6\#12 & $<1000$ & $1 / 16384 \mathrm{C}$ \\
\hline AB5\#7 & $<63$ & $3 / 1024$ & AB6\#4 & $<1000$ & $1 / 4096 \mathrm{C}$ & AB6\#12 & $<1000$ & 1/16384D \\
\hline AB5\#8 & $<1000$ & $1 / 1024 \mathrm{~A}$ & AB6\#4 & $<1000$ & 1/16384B & AB6\#12 & $<63$ & 1/4096B \\
\hline AB5\#8 & $<1000$ & $1 / 1024 \mathrm{~B}$ & AB6\#4 & $<1000$ & $1 / 16384 \mathrm{C}$ & AB6\#13 & $63-1000$ & $1 / 4096 \mathrm{~B}$ \\
\hline AB5\#8 & $<1000$ & 1/4096B & AB6\#4 & $<1000$ & $1 / 16384 \mathrm{D}$ & AB6\#13 & $<1000$ & $1 / 1024 \mathrm{~A}$ \\
\hline AB5\#8 & $<1000$ & $1 / 16384 \mathrm{~A}$ & AB6\#4 & $<63$ & 1/4096B & AB6\#13 & $<1000$ & $1 / 4096 \mathrm{C}$ \\
\hline AB5\#8 & $<1000$ & $1 / 16384 \mathrm{D}$ & AB6\#5 & $63-1000$ & $1 / 4096 \mathrm{~B}$ & AB6\#13 & $<1000$ & 1/16384B \\
\hline AB5\#9 & $<1000$ & $1 / 1024 \mathrm{~A}$ & AB6\#5 & $<1000$ & $1 / 1024 \mathrm{~A}$ & AB6\#13 & $<1000$ & $1 / 16384 C$ \\
\hline AB5\#9 & $<1000$ & 1/1024B & AB6\#5 & $<1000$ & $1 / 4096 \mathrm{C}$ & AB6\#13 & $<1000$ & 1/16384D \\
\hline AB5\#9 & $<1000$ & 1/4096B & AB6\#5 & $<1000$ & 1/16384B & AB6\#13 & $<63$ & $1 / 4096 \mathrm{~B}$ \\
\hline AB5\#9 & $<1000$ & $1 / 16384 \mathrm{~A}$ & AB6\#5 & $<1000$ & $1 / 16384 \mathrm{C}$ & AB7\#1 & $63-1000$ & 1/1024B \\
\hline AB5\#9 & $<1000$ & 1/16384B & AB6\#5 & $<1000$ & $1 / 16384 \mathrm{D}$ & $\mathrm{AB} 7 \# 1$ & $63-1000$ & 1/4096B \\
\hline AB5\#9 & $<1000$ & 1/16384B & AB6\#5 & $<63$ & 1/4096B & AB7\#1 & $<1000$ & $1 / 1024 \mathrm{~A}$ \\
\hline AB5\#9 & $<1000$ & $1 / 16384 \mathrm{D}$ & AB6\#6 & $63-1000$ & 1/4096B & $\mathrm{AB} 7 \# 1$ & $<1000$ & $1 / 4096 \mathrm{C}$ \\
\hline AB5\#10 & $<1000$ & $1 / 1024 \mathrm{~A}$ & AB6\#6 & $<1000$ & $1 / 1024 \mathrm{~A}$ & $\mathrm{AB} 7 \# 1$ & $<1000$ & 1/16384B \\
\hline $\mathrm{AB} 5 \# 10$ & $<1000$ & $1 / 4096 \mathrm{~A}$ & AB6\#6 & $<1000$ & $1 / 4096 \mathrm{C}$ & $\mathrm{AB} 7 \# 1$ & $<1000$ & $1 / 16384 \mathrm{C}$ \\
\hline AB5\#10 & $<1000$ & $1 / 16384 \mathrm{~A}$ & AB6\#6 & $<1000$ & 1/16384B & AB7\#1 & $<1000$ & 1/16384D \\
\hline $\mathrm{AB} 5 \# 10$ & $<1000$ & 1/16384B & AB6\#6 & $<1000$ & $1 / 16384 \mathrm{C}$ & $\mathrm{AB} 7 \# 1$ & $<63$ & $1 / 256 \mathrm{~B}$ \\
\hline AB5\#10 & $<1000$ & $1 / 16384 \mathrm{D}$ & AB6\#6 & $<1000$ & $1 / 16384 \mathrm{D}$ & AB7\#1 & $<63$ & 1/4096B \\
\hline AB5\#11 & $<1000$ & 1/1024B & AB6\#6 & $<63$ & 1/4096B & $\mathrm{AB} 7 \# 2$ & $63-1000$ & 1/1024B \\
\hline AB5\#11 & $<1000$ & 1/4096B & AB6\#7 & $63-1000$ & 1/4096B & $\mathrm{AB} 7 \# 2$ & $63-1000$ & 1/4096B \\
\hline AB5\#11 & $<1000$ & $1 / 16384 \mathrm{~A}$ & AB6\#7 & $<1000$ & $1 / 1024 \mathrm{~A}$ & $\mathrm{AB} 7 \# 2$ & $<1000$ & $1 / 1024 \mathrm{~A}$ \\
\hline AB5\#11 & $<1000$ & $1 / 16384 \mathrm{~B}$ & AB6\#7 & $<1000$ & $1 / 4096 \mathrm{C}$ & $\mathrm{AB} 7 \# 2$ & $<1000$ & $1 / 4096 \mathrm{C}$ \\
\hline AB5\#11 & $<1000$ & 1/16384D & AB6\#7 & $<1000$ & 1/16384B & $\mathrm{AB} 7 \# 2$ & $<1000$ & 1/16384B \\
\hline AB5\#12 & $<1000$ & $1 / 1024 \mathrm{~A}$ & AB6\#7 & $<1000$ & $1 / 16384 \mathrm{C}$ & $\mathrm{AB} 7 \# 2$ & $<1000$ & $1 / 16384 \mathrm{C}$ \\
\hline AB5\#12 & $<1000$ & 1/1024B & AB6\#7 & $<1000$ & 1/16384D & $\mathrm{AB} 7 \# 2$ & $<1000$ & 1/16384D \\
\hline $\mathrm{AB} 5 \# 12$ & $<1000$ & $1 / 1024 \mathrm{C}$ & AB6\#7 & $<63$ & 1/4096B & $\mathrm{AB} 7 \# 2$ & $<63$ & $1 / 256 \mathrm{~B}$ \\
\hline AB5\#12 & $<1000$ & $1 / 16384 \mathrm{~A}$ & AB6\#8 & $63-1000$ & 1/4096B & $\mathrm{AB} 7 \# 2$ & $<63$ & 1/4096B \\
\hline $\mathrm{AB} 5 \# 12$ & $<1000$ & 1/16384B & AB6\#8 & $<1000$ & $1 / 1024 \mathrm{~A}$ & $\mathrm{AB} 7 \# 3$ & $63-1000$ & 1/1024B \\
\hline AB5\#12 & $<1000$ & $1 / 16384 \mathrm{D}$ & AB6\#8 & $<1000$ & $1 / 4096 \mathrm{C}$ & AB7\#3 & $63-1000$ & 1/4096B \\
\hline $\mathrm{AB} 5 \# 12$ & $<63$ & $3 / 1024$ & AB6\#8 & $<1000$ & 1/16384B & $\mathrm{AB} 7 \# 3$ & $<1000$ & $1 / 1024 \mathrm{~A}$ \\
\hline AB5\#13 & $<1000$ & 1/1024B & AB6\#8 & $<1000$ & $1 / 16384 \mathrm{C}$ & $\mathrm{AB} 7 \# 3$ & $<1000$ & $1 / 4096 \mathrm{C}$ \\
\hline AB5\#13 & $<1000$ & $1 / 1024 \mathrm{C}$ & AB6\#8 & $<1000$ & $1 / 16384 \mathrm{D}$ & $\mathrm{AB} 7 \# 3$ & $<1000$ & 1/16384B \\
\hline AB5\#13 & $<1000$ & $1 / 16384 \mathrm{~A}$ & AB6\#8 & $<63$ & 1/4096B & AB7\#3 & $<1000$ & $1 / 16384 \mathrm{C}$ \\
\hline AB5\#13 & $<1000$ & 1/16384B & AB6\#9 & $63-1000$ & $1 / 4096 \mathrm{~B}$ & $\mathrm{AB} 7 \# 3$ & $<1000$ & 1/16384D \\
\hline AB6\#1 & $63-1000$ & $1 / 4096 \mathrm{~A}$ & AB6\#9 & $<1000$ & $1 / 1024 \mathrm{~A}$ & $\mathrm{AB} 7 \# 3$ & $<63$ & $1 / 256 \mathrm{~B}$ \\
\hline AB6\#1 & $<1000$ & $1 / 1024 \mathrm{C}$ & AB6\#9 & $<1000$ & $1 / 4096 \mathrm{C}$ & $\mathrm{AB} 7 \# 3$ & $<63$ & $1 / 4096 \mathrm{~B}$ \\
\hline AB6\#1 & $<1000$ & $1 / 4096 \mathrm{~B}$ & AB6\#9 & $<1000$ & 1/16384B & $\mathrm{AB} 7 \# 4$ & $63-1000$ & 1/1024B \\
\hline
\end{tabular}


Kozo Takahashi, Hirofumi Asahi, Yusuke Okazaki, Jonaotaro Onodera, Hideto Tsutsui,

Takahito Ikenoue, Yoshiyuki Kanematsu, Seiji Tanaka and Shinya Iwasaki

Table 5 (Cont.)

\begin{tabular}{|c|c|c|c|c|c|c|c|c|}
\hline Sample ID & Size $(\mu \mathrm{m})$ & $\begin{array}{l}\text { Aliquot } \\
\text { size \& ID }\end{array}$ & Sample ID & Size $(\mu \mathrm{m})$ & $\begin{array}{l}\text { Aliquot } \\
\text { size \& ID }\end{array}$ & Sample ID & Size $(\mu \mathrm{m})$ & $\begin{array}{l}\text { Aliquot } \\
\text { size \& ID }\end{array}$ \\
\hline AB7\#4 & $63-1000$ & $1 / 4096 \mathrm{~B}$ & $\overline{\mathrm{AB} 7 \# 10}$ & $<1000$ & $1 / 16384 \mathrm{C}$ & $\overline{\mathrm{AB} 8 \# 4}$ & $63-1000$ & $1 / 1024 \mathrm{~B}$ \\
\hline $\mathrm{AB} 7 \# 4$ & $<1000$ & $1 / 1024 \mathrm{~A}$ & $\mathrm{AB} 7 \# 10$ & $<1000$ & $1 / 16384 \mathrm{D}$ & $\mathrm{AB} 8 \# 4$ & $63-1000$ & 1/4096B \\
\hline $\mathrm{AB} 7 \# 4$ & $<1000$ & $1 / 4096 \mathrm{C}$ & $\mathrm{AB} 7 \# 10$ & $<63$ & $1 / 256 \mathrm{~B}$ & AB8\#4 & $<1000$ & $1 / 1024 \mathrm{~A}$ \\
\hline $\mathrm{AB} 7 \# 4$ & $<1000$ & 1/16384B & $\mathrm{AB} 7 \# 10$ & $<63$ & 1/4096B & $\mathrm{AB} 8 \# 4$ & $<1000$ & $1 / 4096 \mathrm{C}$ \\
\hline $\mathrm{AB} 7 \# 4$ & $<1000$ & $1 / 16384 \mathrm{C}$ & AB7\#11 & $63-1000$ & 1/1024B & $\mathrm{AB} 8 \# 4$ & $<1000$ & 1/16384B \\
\hline $\mathrm{AB} 7 \# 4$ & $<1000$ & 1/16384D & AB7\#11 & $63-1000$ & 1/4096B & $\mathrm{AB} 8 \# 4$ & $<1000$ & $1 / 16384 \mathrm{C}$ \\
\hline $\mathrm{AB} 7 \# 4$ & $<63$ & $1 / 256 \mathrm{~B}$ & AB7\#11 & $<1000$ & $1 / 1024 \mathrm{~A}$ & $\mathrm{AB} 8 \# 4$ & $<1000$ & $1 / 16384 \mathrm{D}$ \\
\hline $\mathrm{AB} 7 \# 4$ & $<63$ & $1 / 4096 \mathrm{~B}$ & AB7\#11 & $<1000$ & $1 / 4096 \mathrm{C}$ & $\mathrm{AB} 8 \# 4$ & $<63$ & $1 / 256 \mathrm{~B}$ \\
\hline $\mathrm{AB} 7 \# 5$ & $63-1000$ & 1/1024B & AB7\#11 & $<1000$ & 1/16384B & $\mathrm{AB} 8 \# 4$ & $<63$ & 1/1024B \\
\hline $\mathrm{AB} 7 \# 5$ & $63-1000$ & $1 / 4096 \mathrm{~B}$ & AB7\#11 & $<1000$ & $1 / 16384 \mathrm{C}$ & $\mathrm{AB} 8 \# 5$ & $63-1000$ & 1/1024B \\
\hline $\mathrm{AB} 7 \# 5$ & $<1000$ & $1 / 1024 \mathrm{~A}$ & AB7\#11 & $<1000$ & $1 / 16384 \mathrm{D}$ & $\mathrm{AB} 8 \# 5$ & $63-1000$ & 1/4096B \\
\hline $\mathrm{AB} 7 \# 5$ & $<1000$ & 1/16384B & AB7\#11 & $<63$ & $1 / 256 \mathrm{~B}$ & $\mathrm{AB} 8 \# 5$ & $63-1000$ & $1 / 16384 \mathrm{C}$ \\
\hline $\mathrm{AB} 7 \# 5$ & $<1000$ & $1 / 16384 \mathrm{C}$ & AB7\#11 & $<63$ & 1/4096B & $\mathrm{AB} 8 \# 5$ & $<1000$ & $1 / 1024 \mathrm{~A}$ \\
\hline $\mathrm{AB} 7 \# 5$ & $<1000$ & $1 / 16384 \mathrm{C}$ & $\mathrm{AB} 7 \# 12$ & $63-1000$ & 1/1024B & $\mathrm{AB} 8 \# 5$ & $<1000$ & $1 / 4096 \mathrm{C}$ \\
\hline $\mathrm{AB} 7 \# 5$ & $<1000$ & 1/16384D & $\mathrm{AB} 7 \# 12$ & $63-1000$ & 1/4096B & $\mathrm{AB} 8 \# 5$ & $<1000$ & $1 / 16384 \mathrm{D}$ \\
\hline $\mathrm{AB} 7 \# 5$ & $<63$ & $1 / 256 \mathrm{~B}$ & $\mathrm{AB} 7 \# 12$ & $<1000$ & $1 / 1024 \mathrm{~A}$ & AB8\#5 & $<63$ & $1 / 256 \mathrm{~B}$ \\
\hline $\mathrm{AB} 7 \# 5$ & $<63$ & 1/4096B & AB7\#12 & $<1000$ & $1 / 4096 \mathrm{C}$ & AB8\#5 & $<63$ & 1/1024B \\
\hline AB7\#6 & $63-1000$ & $1 / 1024 \mathrm{~B}$ & $\mathrm{AB} 7 \# 12$ & $<1000$ & 1/16384B & AB8\#5 & $<63$ & $1 / 16384 \mathrm{~B}$ \\
\hline AB7\#6 & $63-1000$ & 1/4096B & $\mathrm{AB} 7 \# 12$ & $<1000$ & $1 / 16384 \mathrm{C}$ & AB8\#6 & $63-1000$ & 1/1024B \\
\hline AB7\#6 & $<1000$ & $1 / 1024 \mathrm{~A}$ & $\mathrm{AB} 7 \# 12$ & $<1000$ & $1 / 16384 \mathrm{D}$ & AB8\#6 & $63-1000$ & 1/4096B \\
\hline AB7\#6 & $<1000$ & $1 / 4096 \mathrm{C}$ & $\mathrm{AB} 7 \# 12$ & $<63$ & $1 / 256 \mathrm{~B}$ & AB8\#6 & $<1000$ & $1 / 1024 \mathrm{~A}$ \\
\hline AB7\#6 & $<1000$ & 1/16384B & $\mathrm{AB} 7 \# 12$ & $<63$ & 1/4096B & AB8\#6 & $<1000$ & $1 / 4096 \mathrm{C}$ \\
\hline AB7\#6 & $<1000$ & $1 / 16384 \mathrm{C}$ & $\mathrm{AB} 7 \# 13$ & $63-1000$ & $1 / 1024 \mathrm{~B}$ & AB8\#6 & $<1000$ & $1 / 16384 \mathrm{~B}$ \\
\hline AB7\#6 & $<1000$ & 1/16384D & AB7\#13 & $63-1000$ & 1/4096B & AB8\#6 & $<1000$ & $1 / 16384 C$ \\
\hline AB7\#6 & $<63$ & $1 / 256 \mathrm{~B}$ & $\mathrm{AB} 7 \# 13$ & $<1000$ & $1 / 1024 \mathrm{~A}$ & AB8\#6 & $<1000$ & $1 / 16384 \mathrm{D}$ \\
\hline AB7\#6 & $<63$ & 1/4096B & $\mathrm{AB} 7 \# 13$ & $<1000$ & $1 / 4096 \mathrm{C}$ & AB8\#6 & $<63$ & $1 / 256 \mathrm{~B}$ \\
\hline AB7\#7 & $63-1000$ & 1/1024B & AB7\#13 & $<1000$ & 1/16384B & AB8\#6 & $<63$ & 1/1024B \\
\hline AB7\#7 & $63-1000$ & 1/4096B & AB7\#13 & $<1000$ & $1 / 16384 \mathrm{C}$ & AB8\#7 & $63-1000$ & 1/1024B \\
\hline AB7\#7 & $<1000$ & $1 / 1024 \mathrm{~A}$ & $\mathrm{AB} 7 \# 13$ & $<63$ & $1 / 256 \mathrm{~B}$ & AB8\#7 & $63-1000$ & 1/4096B \\
\hline $\mathrm{AB} 7 \# 7$ & $<1000$ & $1 / 4096 \mathrm{C}$ & $\mathrm{AB} 7 \# 13$ & $<63$ & 1/4096B & AB8\#7 & $<1000$ & $1 / 1024 \mathrm{~A}$ \\
\hline AB7\#7 & $<1000$ & 1/16384B & AB8\#1 & $63-1000$ & 1/1024B & AB8\#7 & $<1000$ & $1 / 4096 \mathrm{C}$ \\
\hline AB7\#7 & $<1000$ & $1 / 16384 \mathrm{C}$ & AB8\#1 & $63-1000$ & 1/4096B & AB8\#7 & $<1000$ & 1/16384B \\
\hline AB7\#7 & $<1000$ & 1/16384D & AB8\#1 & $<1000$ & $1 / 1024 \mathrm{~A}$ & AB8\#7 & $<1000$ & $1 / 16384 \mathrm{C}$ \\
\hline AB7\#7 & $<63$ & $1 / 256 \mathrm{~B}$ & AB8\#1 & $<1000$ & $1 / 4096 \mathrm{C}$ & AB8\#7 & $<1000$ & $1 / 16384 \mathrm{D}$ \\
\hline $\mathrm{AB} 7 \# 7$ & $<63$ & $1 / 4096 \mathrm{~B}$ & AB8\#1 & $<1000$ & 1/16384B & AB8\#7 & $<63$ & $1 / 256 \mathrm{~B}$ \\
\hline AB7\#8 & $63-1000$ & 1/1024B & AB8\#1 & $<1000$ & $1 / 16384 \mathrm{C}$ & AB8\#7 & $<63$ & $1 / 1024 \mathrm{~A}$ \\
\hline $\mathrm{AB} 7 \# 8$ & $63-1000$ & 1/4096B & AB8\#1 & $<1000$ & 1/16384D & AB8\#8 & $63-1000$ & 1/1024B \\
\hline $\mathrm{AB} 7 \# 8$ & $<1000$ & $1 / 1024 \mathrm{~A}$ & AB8\#1 & $<63$ & $1 / 256 \mathrm{~B}$ & AB8\#8 & $63-1000$ & 1/4096B \\
\hline $\mathrm{AB} 7 \# 8$ & $<1000$ & 1/16384B & AB8\#1 & $<63$ & 1/1024B & AB8\#8 & $<1000$ & $1 / 1024 \mathrm{~A}$ \\
\hline $\mathrm{AB} 7 \# 8$ & $<1000$ & $1 / 16384 \mathrm{C}$ & AB8\#2 & $63-1000$ & 1/1024B & AB8\#8 & $<1000$ & $1 / 4096 \mathrm{C}$ \\
\hline $\mathrm{AB} 7 \# 8$ & $<1000$ & 1/16384D & $\mathrm{AB} 8 \# 2$ & $63-1000$ & 1/4096B & AB8\#8 & $<1000$ & 1/16384B \\
\hline AB7\#8 & $<63$ & $1 / 256 \mathrm{~B}$ & $\mathrm{AB} 8 \# 2$ & $<1000$ & $1 / 1024 \mathrm{~A}$ & $\mathrm{AB} 8 \# 8$ & $<1000$ & $1 / 16384 \mathrm{C}$ \\
\hline AB7\#8 & $<63$ & 1/4096B & $\mathrm{AB} 8 \# 2$ & $<1000$ & $1 / 4096 \mathrm{C}$ & AB8\#8 & $<1000$ & $1 / 16384 \mathrm{D}$ \\
\hline $\mathrm{AB} 7 \# 9$ & $63-1000$ & 1/1024B & AB8\#2 & $<1000$ & 1/16384B & AB8\#8 & $<63$ & $1 / 256 \mathrm{~B}$ \\
\hline AB7\#9 & $63-1000$ & 1/4096B & $\mathrm{AB} 8 \# 2$ & $<1000$ & $1 / 16384 \mathrm{C}$ & AB8\#8 & $<63$ & 1/1024B \\
\hline AB7\#9 & $<1000$ & $1 / 1024 \mathrm{~A}$ & AB8\#2 & $<1000$ & $1 / 16384 \mathrm{D}$ & AB8\#9 & $63-1000$ & $1 / 1024 \mathrm{~B}$ \\
\hline AB7\#9 & $<1000$ & $1 / 4096 \mathrm{C}$ & $\mathrm{AB} 8 \# 2$ & $<63$ & $1 / 256 \mathrm{~B}$ & AB8\#9 & $63-1000$ & $1 / 4096 \mathrm{~B}$ \\
\hline AB7\#9 & $<1000$ & 1/16384B & AB8\#2 & $<63$ & 1/1024B & AB8\#9 & $<1000$ & $1 / 1024 \mathrm{~A}$ \\
\hline AB7\#9 & $<1000$ & $1 / 16384 \mathrm{C}$ & AB8\#3 & $63-1000$ & $1 / 1024 \mathrm{~B}$ & AB8\#9 & $<1000$ & $1 / 4096 \mathrm{C}$ \\
\hline AB7\#9 & $<1000$ & $1 / 16384 \mathrm{D}$ & AB8\#3 & $63-1000$ & 1/4096B & AB8\#9 & $<1000$ & $1 / 16384 \mathrm{~B}$ \\
\hline AB7\#9 & $<63$ & $1 / 256 \mathrm{~B}$ & $\mathrm{AB} 8 \# 3$ & $<1000$ & $1 / 1024 \mathrm{~A}$ & AB8\#9 & $<1000$ & $1 / 16384 \mathrm{C}$ \\
\hline AB7\#9 & $<63$ & $1 / 4096 \mathrm{~B}$ & $\mathrm{AB} 8 \# 3$ & $<1000$ & $1 / 4096 \mathrm{~A}$ & AB8\#9 & $<1000$ & $1 / 16384 \mathrm{D}$ \\
\hline $\mathrm{AB} 7 \# 10$ & $63-1000$ & $1 / 1024 \mathrm{~B}$ & $\mathrm{AB} 8 \# 3$ & $<1000$ & $1 / 16384 \mathrm{~B}$ & AB8\#9 & $<63$ & $1 / 256 \mathrm{~B}$ \\
\hline $\mathrm{AB} 7 \# 10$ & $63-1000$ & $1 / 4096 \mathrm{~B}$ & AB8\#3 & $<1000$ & $1 / 16384 \mathrm{C}$ & AB8\#9 & $<63$ & $1 / 1024 \mathrm{~B}$ \\
\hline $\mathrm{AB} 7 \# 10$ & $<1000$ & $1 / 1024 \mathrm{~A}$ & AB8\#3 & $<1000$ & $1 / 16384 \mathrm{D}$ & AB8\#10 & $63-1000$ & $1 / 1024 \mathrm{~B}$ \\
\hline $\mathrm{AB} 7 \# 10$ & $<1000$ & $1 / 4096 \mathrm{C}$ & $\mathrm{AB} 8 \# 3$ & $<63$ & $1 / 256 \mathrm{~B}$ & AB8\#10 & $63-1000$ & 1/4096B \\
\hline AB7\#10 & $<1000$ & 1/16384B & AB8\#3 & $<63$ & 1/1024B & AB8\#10 & $<1000$ & $1 / 1024 \mathrm{~A}$ \\
\hline
\end{tabular}


Table 5 (Cont.)

\begin{tabular}{|c|c|c|c|c|c|c|c|c|}
\hline Sample ID & Size $(\mu \mathrm{m})$ & $\begin{array}{l}\text { Aliquot } \\
\text { size \& ID }\end{array}$ & Sample ID & Size $(\mu \mathrm{m})$ & $\begin{array}{l}\text { Aliquot } \\
\text { size \& ID }\end{array}$ & Sample ID & Size $(\mu \mathrm{m})$ & $\begin{array}{l}\text { Aliquot } \\
\text { size \& ID }\end{array}$ \\
\hline AB8\#10 & $<1000$ & $1 / 4096 \mathrm{C}$ & $\overline{A B 9 \# 2}$ & $<1000$ & 1/16384D & AB9\#8 & $<1000$ & $1 / 16384 \mathrm{~A}$ \\
\hline AB8\#10 & $<1000$ & 1/16384B & AB9\#2 & $<63$ & $1 / 256 \mathrm{C}$ & AB9\#8 & $<1000$ & 1/16384B \\
\hline AB8\#10 & $<1000$ & $1 / 16384 C$ & AB9\#3 & $63-1000$ & $1 / 1024 \mathrm{C}$ & AB9\#8 & $<1000$ & $1 / 16384 \mathrm{C}$ \\
\hline AB8\#10 & $<1000$ & 1/16384D & AB9\#3 & $63-1000$ & $1 / 4096$ & AB9\#8 & $<1000$ & 1/16384D \\
\hline AB8\#10 & $<63$ & $1 / 256 \mathrm{~B}$ & AB9\#3 & $<1000$ & 1/1024B & AB9\#8 & $<63$ & $1 / 256 \mathrm{C}$ \\
\hline AB8\#10 & $<63$ & $1 / 1024 \mathrm{~B}$ & AB9\#3 & $<1000$ & 1/4096B & AB9\#9 & $63-1000$ & $1 / 1024 \mathrm{C}$ \\
\hline AB8\#11 & $63-1000$ & $1 / 1024 \mathrm{~B}$ & AB9\#3 & $<1000$ & $1 / 16384 \mathrm{~A}$ & AB9\#9 & $63-1000$ & $1 / 4096 \mathrm{C}$ \\
\hline AB8\#11 & $63-1000$ & $1 / 4096 \mathrm{~B}$ & AB9\#3 & $<1000$ & 1/16384B & AB9\#9 & $<1000$ & 1/1024B \\
\hline AB8\#11 & $<1000$ & $1 / 1024 \mathrm{~A}$ & AB9\#3 & $<1000$ & $1 / 16384 \mathrm{C}$ & AB9\#9 & $<1000$ & $1 / 4096 \mathrm{~A}$ \\
\hline AB8\#11 & $<1000$ & $1 / 4096 \mathrm{C}$ & AB9\#3 & $<1000$ & $1 / 16384 \mathrm{D}$ & AB9\#9 & $<1000$ & $1 / 16384 \mathrm{~A}$ \\
\hline AB8\#11 & $<1000$ & $1 / 16384 \mathrm{C}$ & AB9\#3 & $<63$ & $1 / 256 \mathrm{C}$ & AB9\#9 & $<1000$ & 1/16384B \\
\hline AB8\#11 & $<1000$ & 1/16384D & AB9\#4 & $63-1000$ & $1 / 1024 \mathrm{C}$ & AB9\#9 & $<1000$ & 1/16384D \\
\hline AB8\#11 & $<63$ & $1 / 256 \mathrm{~B}$ & AB9\#4 & $63-1000$ & $1 / 4096 \mathrm{C}$ & AB9\#9 & $<63$ & $1 / 256 \mathrm{C}$ \\
\hline AB8\#11 & $<63$ & $1 / 1024 \mathrm{~B}$ & AB9\#4 & $<1000$ & $1 / 1024 \mathrm{~B}$ & AB9\#10 & $63-1000$ & $1 / 1024 \mathrm{C}$ \\
\hline AB8\#12 & $63-1000$ & $1 / 1280 \mathrm{~B}$ & $\mathrm{AB} 9 \# 4$ & $<1000$ & 1/4096B & AB9\#10 & $63-1000$ & $1 / 4096 \mathrm{C}$ \\
\hline AB8\#12 & $63-1000$ & $1 / 5120 \mathrm{~B}$ & AB9\#4 & $<1000$ & $1 / 16384 \mathrm{~A}$ & AB9\#10 & $<1000$ & 1/1024B \\
\hline AB8\#12 & $<1000$ & $1 / 1280 \mathrm{~A}$ & AB9\#4 & $<1000$ & 1/16384B & AB9\#10 & $<1000$ & $1 / 4096 \mathrm{~A}$ \\
\hline AB8\#12 & $<1000$ & $1 / 5120 \mathrm{C}$ & AB9\#4 & $<1000$ & $1 / 16384 \mathrm{C}$ & AB9\#10 & $<1000$ & $1 / 16384 \mathrm{~A}$ \\
\hline AB8\#12 & $<1000$ & $1 / 20480 \mathrm{~B}$ & $\mathrm{AB} 9 \# 4$ & $<1000$ & $1 / 16384 \mathrm{D}$ & AB9\#10 & $<1000$ & 1/16384B \\
\hline AB8\#12 & $<1000$ & $1 / 20480 \mathrm{C}$ & AB9\#4 & $<63$ & $1 / 256 \mathrm{C}$ & AB9\#10 & $<1000$ & $1 / 16384 \mathrm{C}$ \\
\hline AB8\#12 & $<1000$ & $1 / 20480 \mathrm{D}$ & $\mathrm{AB} 9 \# 5$ & $63-1000$ & $1 / 1024 \mathrm{C}$ & AB9\#10 & $<1000$ & 1/16384D \\
\hline AB8\#12 & $<63$ & $1 / 320 \mathrm{C}$ & AB9\#5 & $63-1000$ & $1 / 4096 \mathrm{C}$ & AB9\#10 & $<63$ & $1 / 256 \mathrm{C}$ \\
\hline $\mathrm{AB} 8 \# 12$ & $<63$ & $1 / 5120 \mathrm{~B}$ & $\mathrm{AB} 9 \# 5$ & $<1000$ & $1 / 1024 \mathrm{~B}$ & AB9\#11 & $63-1000$ & $1 / 1024 \mathrm{C}$ \\
\hline AB8\#13 & $63-1000$ & 1/1024B & AB9\#5 & $<1000$ & $1 / 16384 \mathrm{~A}$ & AB9\#11 & $63-1000$ & $1 / 4096 \mathrm{C}$ \\
\hline AB8\#13 & $63-1000$ & $1 / 4096 \mathrm{~B}$ & $\mathrm{AB} 9 \# 5$ & $<1000$ & 1/16384B & AB9\#11 & $<1000$ & 1/1024B \\
\hline $\mathrm{AB} 8 \# 13$ & $<1000$ & $1 / 1024 \mathrm{~A}$ & AB9\#5 & $<1000$ & $1 / 16384 \mathrm{C}$ & AB9\#11 & $<1000$ & 1/4096B \\
\hline AB8\#13 & $<1000$ & $1 / 4096 \mathrm{C}$ & AB9\#5 & $<1000$ & $1 / 16384 \mathrm{D}$ & AB9\#11 & $<1000$ & $1 / 16384$ \\
\hline AB8\#13 & $<1000$ & 1/16384B & AB9\#5 & $<63$ & $1 / 256 \mathrm{C}$ & AB9\#11 & $<1000$ & 1/16384B \\
\hline AB8\#13 & $<1000$ & $1 / 16384 \mathrm{C}$ & AB9\#6 & $63-1000$ & $1 / 1024 \mathrm{C}$ & AB9\#11 & $<1000$ & $1 / 16384 C$ \\
\hline AB8\#13 & $<1000$ & $1 / 16384 \mathrm{D}$ & AB9\#6 & $63-1000$ & $1 / 4096 \mathrm{C}$ & AB9\#11 & $<1000$ & 1/16384D \\
\hline AB8\#13 & $<1000$ & 1/16384D & AB9\#6 & $<1000$ & 1/1024B & AB9\#11 & $<63$ & $1 / 256 \mathrm{C}$ \\
\hline AB8\#13 & $<63$ & $1 / 256 \mathrm{~B}$ & AB9\#6 & $<1000$ & 1/4096B & AB9\#12 & 63-1000 & $1 / 1024 \mathrm{C}$ \\
\hline AB8\#13 & $<63$ & 1/1024B & AB9\#6 & $<1000$ & $1 / 16384 \mathrm{~A}$ & AB9\#12 & $63-1000$ & $1 / 4096 \mathrm{C}$ \\
\hline AB9\#1 & $63-1000$ & $1 / 1024 \mathrm{C}$ & AB9\#6 & $<1000$ & 1/16384B & AB9\#12 & $<1000$ & 1/1024B \\
\hline AB9\#1 & $63-1000$ & $1 / 4096$ & AB9\#6 & $<1000$ & $1 / 16384 \mathrm{C}$ & AB9\#12 & $<1000$ & $1 / 4096 \mathrm{~A}$ \\
\hline AB9\#1 & $<1000$ & 1/1024B & AB9\#6 & $<1000$ & 1/16384D & AB9\#12 & $<1000$ & 1/16384A \\
\hline AB9\#1 & $<1000$ & 1/4096B & AB9\#6 & $<63$ & $1 / 256 \mathrm{C}$ & AB9\#12 & $<1000$ & 1/16384B \\
\hline AB9\#1 & $<1000$ & $1 / 16384 \mathrm{~A}$ & AB9\#7 & $63-1000$ & $1 / 1024 \mathrm{C}$ & AB9\#12 & $<1000$ & $1 / 16384 \mathrm{C}$ \\
\hline AB9\#1 & $<1000$ & 1/16384B & AB9\#7 & $63-1000$ & $1 / 4096 \mathrm{C}$ & AB9\#12 & $<1000$ & 1/16384D \\
\hline AB9\#1 & $<1000$ & $1 / 16384 \mathrm{C}$ & AB9\#7 & $<1000$ & 1/1024B & AB9\#12 & $<63$ & $1 / 256 \mathrm{C}$ \\
\hline AB9\#1 & $<1000$ & $1 / 16384 C$ & AB9\#7 & $<1000$ & 1/4096B & AB9\#13 & $63-1000$ & $1 / 1024 \mathrm{C}$ \\
\hline AB9\#1 & $<1000$ & $1 / 16384 \mathrm{D}$ & AB9\#7 & $<1000$ & $1 / 16384 \mathrm{~A}$ & AB9\#13 & $63-1000$ & $1 / 4096 \mathrm{C}$ \\
\hline AB9\#1 & $<63$ & $1 / 256 \mathrm{C}$ & AB9\#7 & $<1000$ & 1/16384B & AB9\#13 & $<1000$ & $1 / 1024 \mathrm{~B}$ \\
\hline AB9\#2 & $63-1000$ & $1 / 1024 \mathrm{C}$ & AB9\#7 & $<1000$ & $1 / 16384 \mathrm{C}$ & AB9\#13 & $<1000$ & 1/4096B \\
\hline AB9\#2 & $63-1000$ & $1 / 4096$ & AB9\#7 & $<1000$ & $1 / 16384 \mathrm{D}$ & AB9\#13 & $<1000$ & $1 / 16384 \mathrm{~A}$ \\
\hline AB9\#2 & $<1000$ & 1/1024B & AB9\#7 & $<63$ & $1 / 256 \mathrm{C}$ & AB9\#13 & $<1000$ & 1/16384B \\
\hline AB9\#2 & $<1000$ & 1/4096B & AB9\#8 & $63-1000$ & $1 / 1024 \mathrm{C}$ & AB9\#13 & $<1000$ & $1 / 16384 \mathrm{C}$ \\
\hline AB9\#2 & $<1000$ & $1 / 16384 \mathrm{~A}$ & AB9\#8 & $63-1000$ & $1 / 4096 \mathrm{C}$ & AB9\#13 & $<1000$ & 1/16384D \\
\hline AB9\#2 & $<1000$ & 1/16384B & AB9\#8 & $<1000$ & 1/1024B & AB9\#13 & $<63$ & $1 / 256 \mathrm{C}$ \\
\hline AB9\#2 & $<1000$ & $1 / 16384 \mathrm{C}$ & AB9\#8 & $<1000$ & $1 / 4096 \mathrm{C}$ & & & \\
\hline
\end{tabular}


Kozo Takahashi, Hirofumi Asahi, Yusuke Okazaki, Jonaotaro Onodera, Hideto Tsutsui,

Takahito Ikenoue, Yoshiyuki Kanematsu, Seiji Tanaka and Shinya Iwasaki

Table 6. List of archival microslides for Station SA.

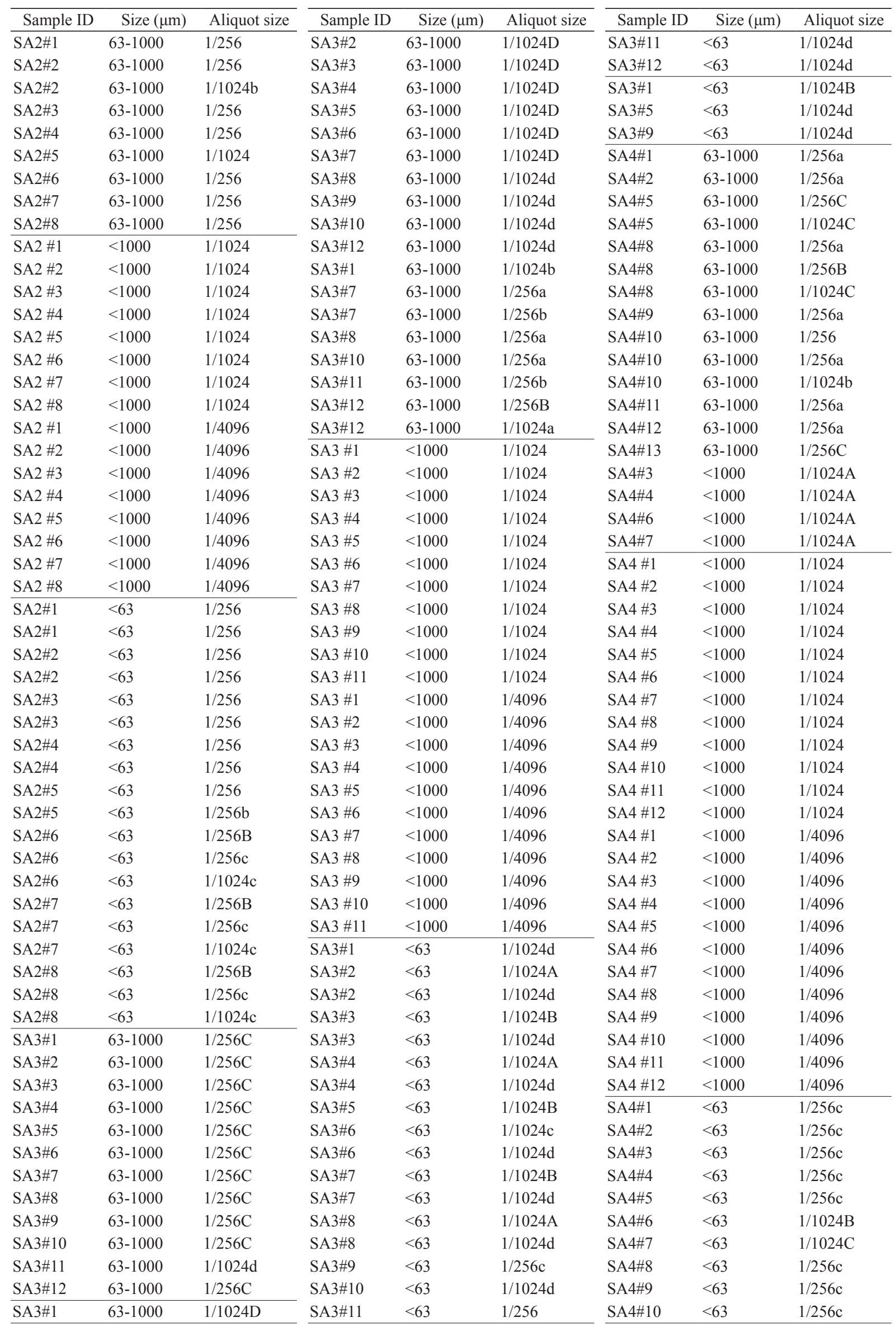


Table 6 (Cont.)

\begin{tabular}{|c|c|c|c|c|c|c|c|c|}
\hline Sample ID & Size $(\mu \mathrm{m})$ & Aliquot size & Sample ID & Size $(\mu \mathrm{m})$ & Aliquot size & Sample ID & Size $(\mu \mathrm{m})$ & Aliquot size \\
\hline SA4\#11 & $<63$ & $1 / 256 c$ & SA5\#9 & $<63$ & $1 / 1024$ & SA7\#5 & $63-1000$ & $1 / 256 \mathrm{~B}$ \\
\hline SA4\#12 & $<63$ & $1 / 256 a$ & SA5\#10 & $<63$ & $1 / 256$ & SA7\#6 & $63-1000$ & $1 / 256 \mathrm{~B}$ \\
\hline SA4\#13 & $<63$ & $1 / 256 \mathrm{~d}$ & SA6\#1 & $63-1000$ & $1 / 256$ & SA7\#7 & $63-1000$ & $1 / 256 \mathrm{~B}$ \\
\hline SA4\#12 & $<63$ & $1 / 1024 \mathrm{~B}$ & SA6\#2 & $63-1000$ & $1 / 256$ & SA7\#8 & $63-1000$ & $1 / 256 \mathrm{~B}$ \\
\hline SA4\#13 & $<63$ & 1/1024B & SA6\#3 & $63-1000$ & $1 / 256$ & SA7\#9 & $63-1000$ & $1 / 256 \mathrm{~B}$ \\
\hline SA4\#1 & $<63$ & $1 / 4096 \mathrm{C}$ & SA6\#4 & $63-1000$ & $1 / 256$ & SA7\#10 & $63-1000$ & $1 / 256 \mathrm{~B}$ \\
\hline SA4\#2 & $<63$ & $1 / 4096 \mathrm{C}$ & SA6\#5 & $63-1000$ & $1 / 256$ & SA7\#11 & $63-1000$ & $1 / 256 \mathrm{~B}$ \\
\hline SA4\#3 & $<63$ & $1 / 4096 \mathrm{C}$ & SA6\#6 & $63-1000$ & $1 / 1024$ & SA7\#12 & $63-1000$ & $1 / 256 \mathrm{~B}$ \\
\hline SA4\#4 & $<63$ & $1 / 4096 \mathrm{C}$ & SA6\#7 & $63-1000$ & $1 / 256$ & SA7\#13 & $63-1000$ & $1 / 256 \mathrm{~B}$ \\
\hline SA4\#5 & $<63$ & $1 / 4096 \mathrm{C}$ & SA6\#8 & $63-1000$ & $1 / 256$ & SA7 \#1 & $<1000$ & $1 / 1024$ \\
\hline SA4\#6 & $<63$ & $1 / 4096 \mathrm{C}$ & SA6\#9 & $63-1000$ & $1 / 256$ & SA7 \#2 & $<1000$ & $1 / 1024$ \\
\hline SA4\#7 & $<63$ & $1 / 4096 \mathrm{C}$ & SA6\#10 & $63-1000$ & $1 / 256$ & $\mathrm{SA} 7$ \#3 & $<1000$ & $1 / 1024$ \\
\hline SA4\#8 & $<63$ & $1 / 4096 \mathrm{C}$ & SA6\#11 & $63-1000$ & $1 / 256$ & SA7 \#4 & $<1000$ & $1 / 1024$ \\
\hline SA4\#9 & $<63$ & $1 / 4096 \mathrm{C}$ & SA6\#12 & $63-1000$ & $1 / 256$ & $\mathrm{SA} 7 \# 5$ & $<1000$ & $1 / 1024$ \\
\hline SA4\#10 & $<63$ & $1 / 4096 \mathrm{C}$ & SA6\#13 & $63-1000$ & $1 / 1024$ & SA 7 \#6 & $<1000$ & $1 / 1024$ \\
\hline SA4\#11 & $<63$ & $1 / 4096 \mathrm{C}$ & SA6 \#1 & $<1000$ & $1 / 1024$ & SA7 \#7 & $<1000$ & $1 / 1024$ \\
\hline SA4\#12 & $<63$ & $1 / 4096 \mathrm{~B}$ & SA6 \#2 & $<1000$ & $1 / 1024$ & SA7 \#8 & $<1000$ & $1 / 1024$ \\
\hline SA4\#13 & $<63$ & 1/4096B & SA6 \#3 & $<1000$ & $1 / 1024$ & SA7 \#9 & $<1000$ & $1 / 1024$ \\
\hline SA4\#12 & $<63$ & $1 / 1024 \mathrm{~B}$ & SA6 \#4 & $<1000$ & $1 / 1024$ & SA7 \#10 & $<1000$ & $1 / 1024$ \\
\hline SA4\#13 & $<63$ & $1 / 1024 \mathrm{~B}$ & SA6 \#5 & $<1000$ & $1 / 1024$ & SA7 \#11 & $<1000$ & $1 / 1024$ \\
\hline SA5\#1 & $63-1000$ & $1 / 256 \mathrm{~A}$ & SA6 \#6 & $<1000$ & $1 / 1024$ & SA7 \#12 & $<1000$ & $1 / 1024$ \\
\hline SA5\#2 & $63-1000$ & $1 / 256 \mathrm{~A}$ & SA6 \#7 & $<1000$ & $1 / 1024$ & SA7 \#13 & $<1000$ & $1 / 1024$ \\
\hline SA5\#3 & $63-1000$ & $1 / 256 \mathrm{~A}$ & SA6 \#8 & $<1000$ & $1 / 1024$ & SA7 \#1 & $<1000$ & $1 / 4096$ \\
\hline SA5\#4 & $63-1000$ & $1 / 256 \mathrm{~A}$ & SA6 \#9 & $<1000$ & $1 / 1024$ & SA7 \#2 & $<1000$ & $1 / 4096$ \\
\hline SA5\#5 & $63-1000$ & $1 / 256 \mathrm{~A}$ & SA6 \#10 & $<1000$ & $1 / 1024$ & SA7 \#3 & $<1000$ & $1 / 4096$ \\
\hline SA5\#6 & $63-1000$ & $1 / 256 \mathrm{~A}$ & SA6 \#11 & $<1000$ & $1 / 1024$ & SA7 \#4 & $<1000$ & $1 / 4096$ \\
\hline SA5\#7 & $63-1000$ & $1 / 256 \mathrm{~A}$ & SA6 \#12 & $<1000$ & $1 / 1024$ & SA7 \#5 & $<1000$ & $1 / 4096$ \\
\hline SA5\#8 & $63-1000$ & $1 / 256 \mathrm{~A}$ & SA6 \#13 & $<1000$ & $1 / 1024$ & SA7 \#6 & $<1000$ & $1 / 4096$ \\
\hline SA5\#9 & $63-1000$ & $1 / 256 \mathrm{~A}$ & SA6 \#1 & $<1000$ & $1 / 4096$ & SA7 \#7 & $<1000$ & $1 / 4096$ \\
\hline SA5\#10 & $63-1000$ & $1 / 256 \mathrm{~A}$ & SA6 \#2 & $<1000$ & $1 / 4096$ & SA7 \#8 & $<1000$ & $1 / 4096$ \\
\hline SA5 \#1 & $<1000$ & $1 / 1024$ & SA6 \#3 & $<1000$ & $1 / 4096$ & SA7 \#9 & $<1000$ & $1 / 4096$ \\
\hline SA 5 \#2 & $<1000$ & $1 / 1024$ & SA6 \#4 & $<1000$ & $1 / 4096$ & SA7 \#10 & $<1000$ & $1 / 4096$ \\
\hline SA5 \#3 & $<1000$ & $1 / 1024$ & SA6 \#5 & $<1000$ & $1 / 4096$ & SA7 \#11 & $<1000$ & $1 / 4096$ \\
\hline SA5 \#4 & $<1000$ & $1 / 1024$ & SA6 \#6 & $<1000$ & $1 / 4096$ & SA7 \#12 & $<1000$ & $1 / 4096$ \\
\hline SA5 \#5 & $<1000$ & $1 / 1024$ & SA6 \#7 & $<1000$ & $1 / 4096$ & SA7 \#13 & $<1000$ & $1 / 4096$ \\
\hline SA5 \#6 & $<1000$ & $1 / 1024$ & SA6 \#8 & $<1000$ & $1 / 4096$ & SA7\#1 & $<63$ & $1 / 256 \mathrm{~B}$ \\
\hline SA5 \#7 & $<1000$ & $1 / 1024$ & SA6 \#9 & $<1000$ & $1 / 4096$ & SA7\#2 & $<63$ & $1 / 256 \mathrm{~B}$ \\
\hline SA5 \#8 & $<1000$ & $1 / 1024$ & SA6 \#10 & $<1000$ & $1 / 4096$ & $\mathrm{SA} 7 \# 3$ & $<63$ & $1 / 256 \mathrm{~B}$ \\
\hline SA5 \#9 & $<1000$ & $1 / 1024$ & SA6 \#11 & $<1000$ & $1 / 4096$ & $\mathrm{SA} 7 \# 4$ & $<63$ & $1 / 256 \mathrm{~B}$ \\
\hline SA5 \#10 & $<1000$ & $1 / 1024$ & SA6 \#12 & $<1000$ & $1 / 4096$ & $\mathrm{SA} 7 \# 5$ & $<63$ & $1 / 256 \mathrm{~B}$ \\
\hline SA5 \#1 & $<1000$ & $1 / 4096$ & SA6 \#13 & $<1000$ & $1 / 4096$ & SA7\#6 & $<63$ & $1 / 256 \mathrm{~B}$ \\
\hline SA5 \#2 & $<1000$ & $1 / 4096$ & $\overline{\text { SA6\#1 }}$ & $<63$ & $1 / 256$ & SA7\#7 & $<63$ & $1 / 256 \mathrm{~B}$ \\
\hline SA5 \#3 & $<1000$ & $1 / 4096$ & SA6\#2 & $<63$ & $1 / 256$ & SA7\#8 & $<63$ & $1 / 256 \mathrm{~B}$ \\
\hline SA5 \#4 & $<1000$ & $1 / 4096$ & SA6\#3 & $<63$ & $1 / 256$ & $\mathrm{SA} 7 \# 9$ & $<63$ & $1 / 256 \mathrm{~B}$ \\
\hline SA5 \#5 & $<1000$ & $1 / 4096$ & SA6\#4 & $<63$ & $2 / 1024$ & SA7\#1 & $<63$ & $1 / 1024 \mathrm{~B}$ \\
\hline SA5 \#6 & $<1000$ & $1 / 4096$ & SA6\#5 & $<63$ & $2 / 4096$ & $\mathrm{SA} 7 \# 2$ & $<63$ & 1/1024B \\
\hline SA5 \#7 & $<1000$ & $1 / 4096$ & SA6\#6 & $<63$ & $1 / 1024$ & $\mathrm{SA} 7 \# 3$ & $<63$ & $1 / 1024 \mathrm{~B}$ \\
\hline SA5 \#8 & $<1000$ & $1 / 4096$ & SA6\#7 & $<63$ & $1 / 256$ & $\mathrm{SA} 7 \# 4$ & $<63$ & $1 / 1024 \mathrm{~B}$ \\
\hline SA5 \#9 & $<1000$ & $1 / 4096$ & SA6\#8 & $<63$ & $1 / 256$ & $\mathrm{SA} 7 \# 5$ & $<63$ & $1 / 1024 \mathrm{~B}$ \\
\hline SA5 \#10 & $<1000$ & $1 / 4096$ & SA6\#9 & $<63$ & $1 / 256$ & $\mathrm{SA} 7 \# 6$ & $<63$ & 1/1024B \\
\hline SA5\#1 & $<63$ & $1 / 256$ & SA6\#10 & $<63$ & $1 / 256$ & SA7\#7 & $<63$ & $1 / 1024 \mathrm{~B}$ \\
\hline SA5\#2 & $<63$ & $1 / 1024$ & SA6\#11 & $<63$ & $1 / 256$ & $\mathrm{SA} 7 \# 8$ & $<63$ & 1/1024B \\
\hline SA5\#3 & $<63$ & $1 / 1024$ & SA6\#12 & $<63$ & $1 / 256$ & SA7\#9 & $<63$ & 1/1024B \\
\hline SA5\#4 & $<63$ & $1 / 1024$ & SA6\#13 & $<63$ & $1 / 4096$ & SA7\#10 & $<63$ & 1/1024B \\
\hline SA5\#5 & $<63$ & $1 / 1024$ & $\overline{\mathrm{SA} 7 \# 1}$ & $63-1000$ & $1 / 256 \mathrm{~B}$ & SA7\#11 & $<63$ & $1 / 1024 \mathrm{~B}$ \\
\hline SA5\#6 & $<63$ & $1 / 1024$ & SA7\#2 & $63-1000$ & $1 / 256 \mathrm{~B}$ & $\mathrm{SA} 7 \# 12$ & $<63$ & $1 / 1024 B$ \\
\hline SA5\#7 & $<63$ & $1 / 256$ & SA7\#3 & $63-1000$ & $1 / 256 \mathrm{~B}$ & $\mathrm{SA} 7 \# 13$ & $<63$ & 1/1024B \\
\hline SA5\#8 & $<63$ & $1 / 256$ & SA7\#4 & $63-1000$ & $1 / 256 \mathrm{~B}$ & SA8\#1 & $63-1000$ & $1 / 256 \mathrm{~B}$ \\
\hline
\end{tabular}


Kozo Takahashi, Hirofumi Asahi, Yusuke Okazaki, Jonaotaro Onodera, Hideto Tsutsui,

Takahito Ikenoue, Yoshiyuki Kanematsu, Seiji Tanaka and Shinya Iwasaki

Table 6 (Cont.)

\begin{tabular}{|c|c|c|c|c|c|c|c|c|}
\hline Sample ID & Size $(\mu \mathrm{m})$ & Aliquot size & Sample ID & Size $(\mu \mathrm{m})$ & Aliquot size & Sample ID & Size $(\mu \mathrm{m})$ & Aliquot size \\
\hline SA8\#2 & $63-1000$ & $1 / 256 \mathrm{~B}$ & SA9\#8 & $63-1000$ & $1 / 256 \mathrm{C}$ & SA10\#13 & $63-1000$ & $1 / 256 \mathrm{C}$ \\
\hline SA8\#3 & $63-1000$ & $1 / 256 \mathrm{~B}$ & SA9\#9 & $63-1000$ & $1 / 256 \mathrm{C}$ & SA10 \#1 & $<1000$ & $1 / 4096$ \\
\hline SA8\#4 & $63-1000$ & $1 / 256 \mathrm{~B}$ & SA9\#10 & $63-1000$ & $1 / 256 \mathrm{C}$ & SA10 \#2 & $<1000$ & $1 / 4096$ \\
\hline SA8\#5 & $63-1000$ & $1 / 256 \mathrm{~B}$ & SA9\#11 & $63-1000$ & $1 / 256 \mathrm{C}$ & SA10 \#3 & $<1000$ & $1 / 4096$ \\
\hline SA8\#6 & $63-1000$ & $1 / 256 \mathrm{~B}$ & SA9\#12 & $63-1000$ & $1 / 256 \mathrm{C}$ & SA10 \#4 & $<1000$ & $1 / 4096$ \\
\hline SA8\#7 & $63-1000$ & $1 / 256 \mathrm{~B}$ & SA9\#13 & $63-1000$ & $1 / 256 \mathrm{C}$ & SA10 \#5 & $<1000$ & $1 / 4096$ \\
\hline SA8\#8 & $63-1000$ & $1 / 256 \mathrm{~B}$ & SA9 \#1 & $<1000$ & $1 / 1024$ & SA10 \#6 & $<1000$ & $1 / 4096$ \\
\hline SA8\#9 & $63-1000$ & $1 / 256 \mathrm{~B}$ & SA9 \#2 & $<1000$ & $1 / 1024$ & SA10 \#7 & $<1000$ & $1 / 4096$ \\
\hline SA8\#10 & $63-1000$ & $1 / 256 \mathrm{~B}$ & SA9 \#3 & $<1000$ & $1 / 1024$ & SA10 \#8 & $<1000$ & $1 / 4096$ \\
\hline SA8\#11 & $63-1000$ & $1 / 256 \mathrm{~B}$ & SA9 \#4 & $<1000$ & $1 / 1024$ & SA10 \#9 & $<1000$ & $1 / 4096$ \\
\hline SA8\#12 & $63-1000$ & $1 / 256 \mathrm{~B}$ & SA9 \#5 & $<1000$ & $1 / 1024$ & SA10 \#10 & $<1000$ & $1 / 4096$ \\
\hline SA8\#13 & $63-1000$ & $1 / 256 \mathrm{~B}$ & SA9 \#6 & $<1000$ & $1 / 1024$ & SA10 \#11 & $<1000$ & $1 / 4096$ \\
\hline SA8 \#1 & $<1000$ & $1 / 1024$ & SA9 \#7 & $<1000$ & $1 / 1024$ & SA10 \#12 & $<1000$ & $1 / 4096$ \\
\hline SA8 \#2 & $<1000$ & $1 / 1024$ & SA9 \#8 & $<1000$ & $1 / 1024$ & SA10 \#13 & $<1000$ & $1 / 4096$ \\
\hline SA8 \#3 & $<1000$ & $1 / 1024$ & SA9 \#9 & $<1000$ & $1 / 1024$ & SA10\#1 & $<63$ & $1 / 256$ \\
\hline SA8 \#4 & $<1000$ & $1 / 1024$ & SA9 \#10 & $<1000$ & $1 / 1024$ & SA10\#1 & $<63$ & $1 / 1024 \mathrm{C}$ \\
\hline SA8 \#5 & $<1000$ & $1 / 1024$ & SA9 \#11 & $<1000$ & $1 / 1024$ & SA10\#1 & $<63$ & $1 / 4096$ \\
\hline SA8 \#6 & $<1000$ & $1 / 1024$ & SA9 \#12 & $<1000$ & $1 / 1024$ & SA10\#2 & $<63$ & $1 / 256$ \\
\hline SA8 \#7 & $<1000$ & $1 / 1024$ & SA9 \#13 & $<1000$ & $1 / 1024$ & SA10\#2 & $<63$ & $1 / 1024 \mathrm{C}$ \\
\hline SA8 \#8 & $<1000$ & $1 / 1024$ & SA9 \#1 & $<1000$ & $1 / 4096$ & SA10\#2 & $<63$ & $1 / 4096$ \\
\hline SA8 \#9 & $<1000$ & $1 / 1024$ & SA9 \#2 & $<1000$ & $1 / 4096$ & SA10\#3 & $<63$ & $1 / 256$ \\
\hline SA8 \#10 & $<1000$ & $1 / 1024$ & SA9 \#3 & $<1000$ & $1 / 4096$ & SA10\#3 & $<63$ & $1 / 1024 \mathrm{C}$ \\
\hline SA8 \#11 & $<1000$ & $1 / 1024$ & SA9 \#4 & $<1000$ & $1 / 4096$ & SA10\#3 & $<63$ & $1 / 4096$ \\
\hline SA8 \#12 & $<1000$ & $1 / 1024$ & SA9 \#5 & $<1000$ & $1 / 4096$ & SA10\#4 & $<63$ & $1 / 256$ \\
\hline SA8 \#13 & $<1000$ & $1 / 1024$ & SA9 \#6 & $<1000$ & $1 / 4096$ & SA10\#4 & $<63$ & $1 / 1024$ \\
\hline SA8 \#1 & $<1000$ & $1 / 4096$ & SA9 \#7 & $<1000$ & $1 / 4096$ & SA10\#4 & $<63$ & $1 / 4096 \mathrm{C}$ \\
\hline SA8 \#2 & $<1000$ & $1 / 4096$ & SA9 \#8 & $<1000$ & $1 / 4096$ & SA10\#5 & $<63$ & $1 / 256$ \\
\hline SA8 \#3 & $<1000$ & $1 / 4096$ & SA9 \#9 & $<1000$ & $1 / 4096$ & SA10\#5 & $<63$ & $1 / 1024$ \\
\hline SA8 \#4 & $<1000$ & $1 / 4096$ & SA9 \#10 & $<1000$ & $1 / 4096$ & SA10\#5 & $<63$ & $1 / 4096 \mathrm{C}$ \\
\hline SA8 \#5 & $<1000$ & $1 / 4096$ & SA9 \#11 & $<1000$ & $1 / 4096$ & SA10\#6 & $<63$ & $1 / 256$ \\
\hline SA8 \#6 & $<1000$ & $1 / 4096$ & SA9 \#12 & $<1000$ & $1 / 4096$ & SA10\#6 & $<63$ & $1 / 1024$ \\
\hline SA8 \#7 & $<1000$ & $1 / 4096$ & SA9 \#13 & $<1000$ & $1 / 4096$ & SA10\#6 & $<63$ & $1 / 4096 \mathrm{C}$ \\
\hline SA8 \#8 & $<1000$ & $1 / 4096$ & SA9\#1 & $<63$ & 1/1024B & SA10\#7 & $<63$ & $1 / 256$ \\
\hline SA8 \#9 & $<1000$ & $1 / 4096$ & SA9\#2 & $<63$ & 1/1024B & SA10\#7 & $<63$ & $1 / 1024$ \\
\hline SA8 \#10 & $<1000$ & $1 / 4096$ & SA9\#3 & $<63$ & 1/1024B & SA10\#7 & $<63$ & $1 / 4096 \mathrm{C}$ \\
\hline SA8 \#11 & $<1000$ & $1 / 4096$ & SA9\#4 & $<63$ & $1 / 1024$ & SA10\#8 & $<63$ & $1 / 256$ \\
\hline SA8 \#12 & $<1000$ & $1 / 4096$ & SA9\#4 & $<63$ & 1/1024B & SA10\#8 & $<63$ & $1 / 1024 \mathrm{C}$ \\
\hline SA8 \#13 & $<1000$ & $1 / 4096$ & SA9\#5 & $<63$ & $1 / 4096 \mathrm{~B}$ & SA10\#8 & $<63$ & $1 / 4096$ \\
\hline SA8\#1 & $<63$ & 1/1024B & SA9\#6 & $<63$ & 1/1024B & SA10\#9 & $<63$ & $1 / 256$ \\
\hline SA8\#2 & $<63$ & 1/1024B & SA9\#7 & $<63$ & 1/4096B & SA10\#9 & $<63$ & $1 / 1024 \mathrm{C}$ \\
\hline SA8\#3 & $<63$ & 1/1024B & SA9\#8 & $<63$ & 1/4096B & SA10\#9 & $<63$ & $1 / 4096$ \\
\hline SA8\#4 & $<63$ & 1/1024B & SA9\#9 & $<63$ & 1/4096B & SA10\#10 & $<63$ & $1 / 256$ \\
\hline SA8\#5 & $<63$ & 1/1024B & SA9\#10 & $<63$ & $1 / 4096 \mathrm{C}$ & SA10\#10 & $<63$ & $1 / 1024 \mathrm{C}$ \\
\hline SA8\#6 & $<63$ & 1/1024B & SA9\#11 & $<63$ & 1/4096B & SA10\#10 & $<63$ & $1 / 4096$ \\
\hline SA8\#7 & $<63$ & 1/1024B & SA9\#12 & $<63$ & 1/4096B & SA10\#11 & $<63$ & $1 / 256$ \\
\hline SA8\#8 & $<63$ & 1/1024B & SA9\#13 & $<63$ & 1/4096B & SA10\#11 & $<63$ & $1 / 1024 \mathrm{C}$ \\
\hline SA8\#9 & $<63$ & 1/1024B & $\overline{\text { SA10\#1 }}$ & $63-1000$ & $1 / 256 \mathrm{C}$ & SA10\#11 & $<63$ & $1 / 4096$ \\
\hline SA8\#10 & $<63$ & 1/1024B & SA10\#2 & $63-1000$ & $1 / 256 \mathrm{C}$ & SA10\#12 & $<63$ & $1 / 256$ \\
\hline SA8\#11 & $<63$ & 1/1024B & SA10\#3 & $63-1000$ & $1 / 256 \mathrm{C}$ & SA10\#12 & $<63$ & $1 / 1024 C$ \\
\hline SA8\#12 & $<63$ & 1/1024B & SA10\#4 & $63-1000$ & $1 / 256 \mathrm{C}$ & SA10\#12 & $<63$ & $1 / 4096$ \\
\hline SA8\#13 & $<63$ & 1/1024B & SA10\#5 & $63-1000$ & $1 / 256 \mathrm{C}$ & SA10\#13 & $<63$ & $1 / 256$ \\
\hline SA9\#1 & $63-1000$ & $1 / 256 \mathrm{C}$ & SA10\#6 & $63-1000$ & $1 / 256 \mathrm{C}$ & SA10\#13 & $<63$ & $1 / 1024$ \\
\hline SA9\#2 & $63-1000$ & $1 / 256 \mathrm{C}$ & SA10\#7 & $63-1000$ & $1 / 256 \mathrm{C}$ & SA10\#13 & $<63$ & $1 / 4096 \mathrm{C}$ \\
\hline SA9\#3 & $63-1000$ & $1 / 256 \mathrm{C}$ & SA10\#8 & $63-1000$ & $1 / 256 \mathrm{C}$ & SA11\#1 & $63-1000$ & $1 / 256$ \\
\hline SA9\#4 & $63-1000$ & $1 / 1024$ & SA10\#9 & $63-1000$ & $1 / 256 \mathrm{C}$ & SA11\#2 & $63-1000$ & $1 / 256$ \\
\hline SA9\#5 & $63-1000$ & $1 / 256 \mathrm{C}$ & SA10\#10 & $63-1000$ & $1 / 256 \mathrm{C}$ & SA11\#3 & $63-1000$ & $1 / 256$ \\
\hline SA9\#6 & $63-1000$ & $1 / 256 \mathrm{C}$ & SA10\#11 & $63-1000$ & $1 / 256 \mathrm{C}$ & SA11\#4 & $63-1000$ & $1 / 256$ \\
\hline SA9\#7 & $63-1000$ & $1 / 256 \mathrm{C}$ & SA10\#12 & $63-1000$ & $1 / 256 \mathrm{C}$ & SA11\#5 & $63-1000$ & $1 / 256$ \\
\hline
\end{tabular}


Table 6 (Cont.)

\begin{tabular}{|c|c|c|c|c|c|c|c|c|}
\hline Sample ID & Size $(\mu \mathrm{m})$ & Aliquot size & Sample ID & Size $(\mu \mathrm{m})$ & Aliquot size & Sample ID & Size $(\mu \mathrm{m})$ & Aliquot size \\
\hline SA11\#6 & $63-1000$ & $1 / 256$ & SA12 \#12 & $<1000$ & $1 / 4096$ & SA14 \#5 & $<1000$ & $1 / 4096$ \\
\hline SA11\#7 & $63-1000$ & $1 / 256$ & SA12 \#13 & $<1000$ & $1 / 4096$ & SA14 \#6 & $<1000$ & $1 / 4096$ \\
\hline SA11\#8 & $63-1000$ & $1 / 256$ & SA12\#1 & $<63$ & $1 / 320$ & SA14 \#7 & $<1000$ & $1 / 4096$ \\
\hline SA11\#9 & $63-1000$ & $1 / 256$ & SA12\#2 & $<63$ & $1 / 320$ & SA14 \#8 & $<1000$ & $1 / 4096$ \\
\hline SA11\#10 & $63-1000$ & $1 / 256$ & SA12\#3 & $<63$ & $1 / 1280$ & SA14 \#9 & $<1000$ & $1 / 4096$ \\
\hline SA11\#11 & $63-1000$ & $1 / 256$ & SA12\#4 & $<63$ & $1 / 320$ & SA14 \#10 & $<1000$ & $1 / 4096$ \\
\hline SA11\#12 & $63-1000$ & $1 / 256$ & SA12\#5 & $<63$ & $1 / 320$ & SA14 \#11 & $<1000$ & $1 / 4096$ \\
\hline SA11\#13 & $63-1000$ & $1 / 256$ & SA12\#6 & $<63$ & $1 / 320$ & SA14 \#12 & $<1000$ & $1 / 4096$ \\
\hline SA11 \#1 & $<1000$ & $1 / 4096$ & SA12\#7 & $<63$ & $1 / 320$ & SA14\#1 & $<63$ & $1 / 64$ \\
\hline SA11 \#2 & $<1000$ & $1 / 4096$ & SA12\#8 & $<63$ & $1 / 320$ & SA14\#2 & $<63$ & $1 / 64$ \\
\hline SA11 \#3 & $<1000$ & $1 / 4096$ & SA12\#9 & $<63$ & $1 / 320$ & SA14\#3 & $<63$ & $1 / 64$ \\
\hline SA11 \#4 & $<1000$ & $1 / 4096$ & SA12\#10 & $<63$ & $1 / 320$ & SA14\#4 & $<63$ & $1 / 64$ \\
\hline SA11 \#5 & $<1000$ & $1 / 4096$ & SA12\#11 & $<63$ & $1 / 320$ & SA14\#5 & $<63$ & $1 / 256$ \\
\hline SA11 \#6 & $<1000$ & $1 / 4096$ & SA12\#12 & $<63$ & $1 / 320$ & SA14\#6 & $<63$ & $1 / 256$ \\
\hline SA11 \#7 & $<1000$ & $1 / 4096$ & SA12\#13 & $<63$ & $1 / 320$ & SA14\#7 & $<63$ & $1 / 64$ \\
\hline SA11 \#8 & $<1000$ & $1 / 4096$ & SA13\#1 & $63-1000$ & $1 / 256$ & SA14\#8 & $<63$ & $1 / 64$ \\
\hline SA11 \#9 & $<1000$ & $1 / 4096$ & SA13\#2 & $63-1000$ & $1 / 256$ & SA14\#9 & $<63$ & $1 / 64$ \\
\hline SA11 \#10 & $<1000$ & $1 / 4096$ & SA13\#3 & $63-1000$ & $1 / 64$ & SA14\#10 & $<63$ & $1 / 64$ \\
\hline SA11 \#11 & $<1000$ & $1 / 4096$ & SA13\#4 & $63-1000$ & $1 / 64$ & SA14\#11 & $<63$ & $1 / 64$ \\
\hline SA11 \#12 & $<1000$ & $1 / 4096$ & SA13\#5 & $63-1000$ & $1 / 256$ & SA14\#12 & $<63$ & $1 / 16$ \\
\hline SA11 \#13 & $<1000$ & $1 / 4096$ & SA13\#6 & $63-1000$ & $1 / 256$ & SA14\#13 & $<63$ & $1 / 16$ \\
\hline SA11\#1 & $<63$ & $1 / 256$ & SA13\#7 & $63-1000$ & $1 / 64$ & SA15\#1 & $63-1000$ & $1 / 256$ \\
\hline SA11\#2 & $<63$ & $1 / 256$ & SA13\#8 & $63-1000$ & $1 / 64$ & SA15\#2 & $63-1000$ & $1 / 256$ \\
\hline SA11\#3 & $<63$ & $1 / 256$ & SA13\#9 & $63-1000$ & $1 / 64$ & SA15\#3 & $63-1000$ & $1 / 256$ \\
\hline SA11\#4 & $<63$ & $1 / 256$ & SA13\#10 & $63-1000$ & $1 / 256$ & SA15\#4 & $63-1000$ & $1 / 64$ \\
\hline SA11\#5 & $<63$ & $1 / 256$ & SA13\#11 & $63-1000$ & $1 / 64$ & SA15\#5 & $63-1000$ & $1 / 64$ \\
\hline SA11\#6 & $<63$ & $1 / 256$ & SA13\#12 & $63-1000$ & $1 / 64$ & SA15\#6 & $63-1000$ & $1 / 256$ \\
\hline SA11\#7 & $<63$ & $1 / 256$ & SA13\#13 & $63-1000$ & $1 / 256$ & SA15\#7 & $63-1000$ & $1 / 64$ \\
\hline SA11\#8 & $<63$ & $1 / 256$ & SA13\#1 & $<63$ & $1 / 1280$ & SA15\#8 & $63-1000$ & $1 / 64$ \\
\hline SA11\#9 & $<63$ & $1 / 256$ & SA13\#2 & $<63$ & $1 / 1280$ & SA15\#9 & $63-1000$ & $1 / 64$ \\
\hline SA11\#10 & $<63$ & $1 / 256$ & SA13\#3 & $<63$ & $1 / 320$ & SA15\#10 & $63-1000$ & $1 / 64$ \\
\hline SA11\#11 & $<63$ & $1 / 256$ & SA13\#4 & $<63$ & $1 / 320$ & SA15 \#1 & $<1000$ & $1 / 4096$ \\
\hline SA11\#12 & $<63$ & $1 / 256$ & SA13\#5 & $<63$ & $1 / 1280$ & SA15 \#2 & $<1000$ & $1 / 4096$ \\
\hline SA11\#13 & $<63$ & $1 / 256$ & SA13\#6 & $<63$ & $1 / 1280$ & SA15 \#3 & $<1000$ & $1 / 4096$ \\
\hline SA12\#1 & $63-1000$ & $1 / 64$ & SA13\#7 & $<63$ & $1 / 320$ & SA15 \#4 & $<1000$ & $1 / 4096$ \\
\hline $\mathrm{SA} 12 \# 2$ & $63-1000$ & $1 / 64$ & SA13\#8 & $<63$ & $1 / 320$ & SA $15 \# 5$ & $<1000$ & $1 / 4096$ \\
\hline SA12\#3 & $63-1000$ & $1 / 256$ & SA13\#9 & $<63$ & $1 / 320$ & SA15 \#6 & $<1000$ & $1 / 4096$ \\
\hline SA12\#4 & $63-1000$ & $1 / 64$ & SA13\#10 & $<63$ & $1 / 1280$ & SA15 \#7 & $<1000$ & $1 / 4096$ \\
\hline SA12\#5 & $63-1000$ & $1 / 64$ & SA13\#11 & $<63$ & $1 / 320$ & SA15 \#8 & $<1000$ & $1 / 4096$ \\
\hline SA12\#6 & $63-1000$ & $1 / 64$ & SA13\#12 & $<63$ & $1 / 320$ & SA15 \#9 & $<1000$ & $1 / 4096$ \\
\hline SA12\#7 & $63-1000$ & $1 / 64$ & SA13\#13 & $<63$ & $1 / 1280$ & SA15 \#10 & $<1000$ & $1 / 4096$ \\
\hline SA $12 \# 8$ & $63-1000$ & $1 / 64$ & SA14\#1 & $63-1000$ & $1 / 64$ & SA15\#1 & $<63$ & $1 / 1280$ \\
\hline SA12\#9 & $63-1000$ & $1 / 64$ & SA14\#2 & $63-1000$ & $1 / 64$ & SA $15 \# 2$ & $<63$ & $1 / 1280$ \\
\hline SA $12 \# 10$ & $63-1000$ & $1 / 64$ & SA14\#3 & $63-1000$ & $1 / 64$ & SA15\#3 & $<63$ & $1 / 1280$ \\
\hline SA12\#11 & $63-1000$ & $1 / 64$ & SA14\#4 & $63-1000$ & $1 / 64$ & SA15\#4 & $<63$ & $1 / 320$ \\
\hline SA12\#12 & $63-1000$ & $1 / 64$ & SA14\#5 & $63-1000$ & $1 / 256$ & SA15\#5 & $<63$ & $1 / 320$ \\
\hline SA12\#13 & $63-1000$ & $1 / 64$ & SA14\#6 & $63-1000$ & $1 / 256$ & SA15\#6 & $<63$ & $1 / 1280$ \\
\hline SA12 \#1 & $<1000$ & $1 / 4096$ & SA14\#7 & $63-1000$ & $1 / 64$ & SA15\#7 & $<63$ & $1 / 320$ \\
\hline SA12 \#2 & $<1000$ & $1 / 4096$ & SA14\#8 & $63-1000$ & $1 / 64$ & SA $15 \# 8$ & $<63$ & $1 / 64$ \\
\hline SA12 \#3 & $<1000$ & $1 / 4096$ & SA14\#9 & $63-1000$ & $1 / 64$ & SA15\#9 & $<63$ & $1 / 320$ \\
\hline SA12 \#4 & $<1000$ & $1 / 4096$ & SA14\#10 & $63-1000$ & $1 / 64$ & SA15\#10 & $<63$ & $1 / 320$ \\
\hline SA12 \#5 & $<1000$ & $1 / 4096$ & SA14\#11 & $63-1000$ & $1 / 64$ & SA16\#1 & $63-1000$ & $1 / 64$ \\
\hline SA12 \#6 & $<1000$ & $1 / 4096$ & SA14\#12 & $63-1000$ & $1 / 16$ & SA16\#2 & $63-1000$ & $1 / 64$ \\
\hline SA12 \#7 & $<1000$ & $1 / 4096$ & SA14\#13 & $63-1000$ & $1 / 16$ & SA16\#3 & $63-1000$ & $1 / 64$ \\
\hline SA12 \#8 & $<1000$ & $1 / 4096$ & $\overline{\text { SA14 \#1 }}$ & $<1000$ & $1 / 4096$ & SA16\#4 & $63-1000$ & $1 / 256$ \\
\hline SA12 \#9 & $<1000$ & $1 / 4096$ & SA14 \#2 & $<1000$ & $1 / 4096$ & SA16\#5-1 & $63-1000$ & $1 / 256$ \\
\hline SA12 \#10 & $<1000$ & $1 / 4096$ & SA14 \#3 & $<1000$ & $1 / 4096$ & SA16\#5-2 & $63-1000$ & $1 / 256$ \\
\hline SA12 \#11 & $<1000$ & $1 / 4096$ & SA14 \#4 & $<1000$ & $1 / 4096$ & SA16\#5-3 1/2 & $63-1000$ & $1 / 64$ \\
\hline
\end{tabular}


Kozo Takahashi, Hirofumi Asahi, Yusuke Okazaki, Jonaotaro Onodera, Hideto Tsutsui,

Takahito Ikenoue, Yoshiyuki Kanematsu, Seiji Tanaka and Shinya Iwasaki

Table 6 (Cont.)

\begin{tabular}{|c|c|c|c|c|c|c|c|c|}
\hline Sample ID & Size $(\mu \mathrm{m})$ & Aliquot size & $\begin{array}{c}\text { Sample ID } \\
\end{array}$ & Size $(\mu \mathrm{m})$ & Aliquot size & Sample ID & Size $(\mu \mathrm{m})$ & Aliquot size \\
\hline SA16\#5-3 2/2 & $63-1000$ & $1 / 64$ & SA17\#8 & $63-1000$ & $1 / 256$ & SA19 \#3 & $<1000$ & $1 / 4096$ \\
\hline SA16\#5-4 & $63-1000$ & $1 / 64$ & SA17\#9 & $63-1000$ & $1 / 256$ & SA19 \#4 & $<1000$ & $1 / 4096$ \\
\hline SA16\#6 & $63-1000$ & $1 / 256$ & SA17\#10 & $63-1000$ & $1 / 256$ & SA19 \#5 & $<1000$ & $1 / 4096$ \\
\hline SA16\#7 & $63-1000$ & $1 / 64$ & SA17\#11 & $63-1000$ & $1 / 256$ & SA19 \#6 & $<1000$ & $1 / 4096$ \\
\hline SA16\#8 & $63-1000$ & $1 / 64$ & SA17\#13 & $63-1000$ & $1 / 256$ & SA19 \#7 & $<1000$ & $1 / 4096$ \\
\hline SA16\#9 1/2 & $63-1000$ & $1 / 64$ & SA17\#14 & $63-1000$ & $1 / 256$ & SA19 \#8 & $<1000$ & $1 / 4096$ \\
\hline SA $16 \# 9$ 2/2 & $63-1000$ & $1 / 64$ & SA17\#15 & $63-1000$ & $1 / 256$ & SA19 \#9 & $<1000$ & $1 / 4096$ \\
\hline SA16\#10 1/2 & $63-1000$ & $1 / 64$ & SA17\#16 & $63-1000$ & $1 / 256$ & SA19 \#10 & $<1000$ & $1 / 4096$ \\
\hline SA16\#10 2/2 & $63-1000$ & $1 / 64$ & SA17\#17 & $63-1000$ & $1 / 256$ & SA19 \#11 & $<1000$ & $1 / 4096$ \\
\hline SA16\#11 & $63-1000$ & $1 / 64$ & SA17\#18 & $63-1000$ & $1 / 256$ & SA19 \#12 & $<1000$ & $1 / 4096$ \\
\hline SA16\#12 & $63-1000$ & $1 / 64$ & SA17\#19 & $63-1000$ & $1 / 256$ & SA19 \#13 & $<1000$ & $1 / 4096$ \\
\hline SA16\#13 & $63-1000$ & $1 / 64$ & SA17\#20 & $63-1000$ & $1 / 256$ & SA19 \#14 & $<1000$ & $1 / 4096$ \\
\hline SA16 \#1 & $<1000$ & $1 / 4096$ & SA17\#21 & $63-1000$ & $1 / 256$ & SA19 \#15 & $<1000$ & $1 / 4096$ \\
\hline SA16 \#2 & $<1000$ & $1 / 4096$ & SA17 \#1 & $<1000$ & $1 / 4096$ & SA19 \#16 & $<1000$ & $1 / 4096$ \\
\hline SA16 \#3 & $<1000$ & $1 / 4096$ & SA17 \#2 & $<1000$ & $1 / 4096$ & SA19 \#17 & $<1000$ & $1 / 4096$ \\
\hline SA16 \#4 & $<1000$ & $1 / 4096$ & SA17 \#3 & $<1000$ & $1 / 4096$ & SA19 \#18 & $<1000$ & $1 / 4096$ \\
\hline SA16 \#5 & $<1000$ & $1 / 4096$ & SA17 \#4 & $<1000$ & $1 / 4096$ & SA19 \#19 & $<1000$ & $1 / 4096$ \\
\hline SA16 \#6 & $<1000$ & $1 / 4096$ & SA17 \#5 & $<1000$ & $1 / 4096$ & SA19 \#20 & $<1000$ & $1 / 4096$ \\
\hline SA16 \#7 & $<1000$ & $1 / 4096$ & SA17 \#6 & $<1000$ & $1 / 4096$ & SA19 \#21 & $<1000$ & $1 / 4096$ \\
\hline SA16 \#8 & $<1000$ & $1 / 4096$ & SA17 \#7 & $<1000$ & $1 / 4096$ & SA2\#2 & $63-125$ & $1 / 64$ \\
\hline SA16 \#9 & $<1000$ & $1 / 4096$ & SA17 \#8 & $<1000$ & $1 / 4096$ & SA2\#4 & $63-125$ & $1 / 64$ \\
\hline SA16 \#10 & $<1000$ & $1 / 4096$ & SA17 \#9 & $<1000$ & $1 / 4096$ & SA2\#5 & $63-125$ & $1 / 64$ \\
\hline SA16 \#11 & $<1000$ & $1 / 4096$ & SA17 \#10 & $<1000$ & $1 / 4096$ & SA2\#6 & $63-125$ & $1 / 64$ \\
\hline SA16 \#12 & $<1000$ & $1 / 4096$ & SA17 \#11 & $<1000$ & $1 / 4096$ & SA2\#8 & $63-125$ & $1 / 64$ \\
\hline SA16 \#13 & $<1000$ & $1 / 4096$ & SA17 \#12 & $<1000$ & $1 / 4096$ & SA3\#2 & $63-125$ & $1 / 64$ \\
\hline SA16\#1 & $<63$ & $1 / 320$ & SA17 \#13 & $<1000$ & $1 / 4096$ & SA3\#3 & $63-125$ & $1 / 64$ \\
\hline SA16\#2 & $<63$ & $1 / 320$ & SA17 \#14 & $<1000$ & $1 / 4096$ & SA3\#4 & $63-125$ & $1 / 64$ \\
\hline SA16\#3 & $<63$ & $1 / 320$ & SA17 \#15 & $<1000$ & $1 / 4096$ & SA3\#5 & $63-125$ & $1 / 64$ \\
\hline SA16\#4 & $<63$ & $1 / 1280$ & SA17 \#16 & $<1000$ & $1 / 4096$ & SA3\#8 & $63-125$ & $1 / 64$ \\
\hline SA16\#5-1 & $<63$ & $1 / 1280$ & SA17 \#17 & $<1000$ & $1 / 4096$ & SA3\#11 & $63-125$ & $1 / 64$ \\
\hline SA16\#5-2 & $<63$ & $1 / 1280$ & SA17 \#18 & $<1000$ & $1 / 4096$ & SA4\#1 & $63-125$ & $1 / 64$ \\
\hline SA16\#5-3 & $<63$ & $1 / 320$ & SA17 \#19 & $<1000$ & $1 / 4096$ & SA4\#4 & $63-125$ & $1 / 64$ \\
\hline SA16\#5-4 & $<63$ & $1 / 320$ & SA17 \#20 & $<1000$ & $1 / 4096$ & SA4\#6 & $63-125$ & $1 / 64$ \\
\hline SA16\#6 & $<63$ & $1 / 1280$ & SA17 \#21 & $<1000$ & $1 / 4096$ & SA4\#8 & $63-125$ & $1 / 64$ \\
\hline SA16\#7 & $<63$ & $1 / 160$ & SA18 \#1 & $<1000$ & $1 / 4096$ & SA4\#9 & $63-125$ & $1 / 64$ \\
\hline SA16\#8 & $<63$ & $1 / 320$ & SA18 \#2 & $<1000$ & $1 / 4096$ & SA4\#10 & $63-125$ & $1 / 64$ \\
\hline SA16\#9 & $<63$ & $1 / 320$ & SA18 \#3 & $<1000$ & $1 / 4096$ & SA4\#11 & $63-125$ & $1 / 64$ \\
\hline SA16\#10 & $<63$ & $1 / 320$ & SA18 \#4 & $<1000$ & $1 / 4096$ & $\overline{S A 5 \# 1}$ & $63-125$ & $1 / 64$ \\
\hline SA16\#11 & $<63$ & $1 / 320$ & SA18 \#5 & $<1000$ & $1 / 4096$ & SA5\#2 & $63-125$ & $1 / 64$ \\
\hline SA16\#12 & $<63$ & $1 / 320$ & SA18 \#6 & $<1000$ & $1 / 4096$ & SA5\#3 & $63-125$ & $1 / 64$ \\
\hline SA16\#13 & $<63$ & $1 / 320$ & SA18 \#7 & $<1000$ & $1 / 4096$ & SA5\#4 & $63-125$ & $1 / 64$ \\
\hline SA17\#1 & $63-1000$ & $1 / 256$ & SA18 \#8 & $<1000$ & $1 / 4096$ & SA5\#5 & $63-125$ & $1 / 64$ \\
\hline SA17\#2 & $63-1000$ & $1 / 256$ & SA18 \#9 & $<1000$ & $1 / 4096$ & SA5\#6+\#7 & $63-125$ & $1 / 64$ \\
\hline SA17\#3 & $63-1000$ & $1 / 256$ & SA18 \#10 & $<1000$ & $1 / 4096$ & SA5\#8 & $63-125$ & $1 / 64$ \\
\hline SA17\#4 & $63-1000$ & $1 / 256$ & SA18 \#11 & $<1000$ & $1 / 4096$ & SA5\#9 & $63-125$ & $1 / 64$ \\
\hline SA17\#5 & $63-1000$ & $1 / 256$ & SA18 \#12 & $<1000$ & $1 / 4096$ & SA5\#10 & $63-125$ & $1 / 64$ \\
\hline SA17\#6 & $63-1000$ & $1 / 256$ & $\overline{\text { SA19 \#1 }}$ & $<1000$ & $1 / 4096$ & & & \\
\hline SA17\#7 & $63-1000$ & $1 / 256$ & SA19 \#2 & $<1000$ & $1 / 4096$ & & & \\
\hline
\end{tabular}

Table 7. List of archival microslides for Station AB.

\begin{tabular}{|c|c|c|c|c|c|c|c|c|}
\hline Sample ID & Size $(\mu \mathrm{m})$ & Aliquot size & Sample ID & Size $(\mu \mathrm{m})$ & Aliquot size & Sample ID & Size $(\mu \mathrm{m})$ & Aliquot size \\
\hline $\mathrm{AB} 1 \# 1$ & $63-1000$ & 1/1024B & $\mathrm{AB} 1 \# 5$ & $63-1000$ & 1/1024B & $\overline{\mathrm{AB} 1 \# 10}$ & $63-1000$ & 1/1024B \\
\hline $\mathrm{AB} 1 \# 2$ & $63-1000$ & 1/1024B & $\mathrm{AB} 1 \# 6$ & $63-1000$ & 1/1024B & AB1\#11 & $63-1000$ & 1/1024B \\
\hline $\mathrm{AB} 1 \# 3$ & $63-1000$ & 1/1024B & $\mathrm{AB} 1 \# 7$ & $63-1000$ & 1/1024B & $\mathrm{AB} 1 \# 12$ & $63-1000$ & $1 / 256 b$ \\
\hline $\mathrm{AB} 1 \# 4$ & $63-1000$ & $1 / 1024 a$ & $\mathrm{AB} 1 \# 8$ & $63-1000$ & 1/1024B & $\mathrm{AB} 1 \# 12$ & $63-1000$ & $1 / 256 \mathrm{c}$ \\
\hline $\mathrm{AB} 1 \# 4$ & $63-1000$ & $1 / 1024 \mathrm{c}$ & $\mathrm{AB} 1 \# 9$ & $63-1000$ & 1/1024B & $\overline{\mathrm{AB} 1 \# 1}$ & $<1000$ & $1 / 1024$ \\
\hline
\end{tabular}


Table 7 (Cont.)

\begin{tabular}{|c|c|c|c|c|c|c|c|c|}
\hline Sample ID & Size $(\mu \mathrm{m})$ & Aliquot size & Sample ID & Size $(\mu \mathrm{m})$ & Aliquot size & Sample ID & Size $(\mu \mathrm{m})$ & Aliquot size \\
\hline $\mathrm{AB} 1 \# 2$ & $<1000$ & $1 / 1024$ & $\mathrm{AB} 2 \# 5$ & $<1000$ & $1 / 4096$ & $\mathrm{AB} 3 \# 1$ & $<63$ & 1/4096D \\
\hline $\mathrm{AB} 1 \# 3$ & $<1000$ & $1 / 1024$ & $\mathrm{AB} 2 \# 6$ & $<1000$ & $1 / 4096$ & AB3\#1 & $<63$ & $1 / 12288 \mathrm{c}$ \\
\hline $\mathrm{AB} 1 \# 4$ & $<1000$ & $1 / 1024$ & $\mathrm{AB} 2 \# 7$ & $<1000$ & $1 / 4096$ & AB3\#2 & $<63$ & 1/4096D \\
\hline $\mathrm{AB} 1 \# 5$ & $<1000$ & $1 / 1024$ & $\mathrm{AB} 2 \# 8$ & $<1000$ & $1 / 4096$ & AB3\#2 & $<63$ & $1 / 12288 b$ \\
\hline $\mathrm{AB} 1 \# 6$ & $<1000$ & $1 / 1024$ & $\mathrm{AB} 2 \# 9$ & $<1000$ & $1 / 4096$ & AB3\#4 & $<63$ & $1 / 1024$ \\
\hline $\mathrm{AB} 1 \# 7$ & $<1000$ & $1 / 1024$ & AB2\#10 & $<1000$ & $1 / 4096$ & AB3\#4 & $<63$ & $1 / 1024 d$ \\
\hline $\mathrm{AB} 1 \# 8$ & $<1000$ & $1 / 1024$ & AB2\#11 & $<1000$ & $1 / 4096$ & AB3\#9 & $<63$ & $1 / 1024 a$ \\
\hline AB1\#9 & $<1000$ & $1 / 1024$ & AB2\#12 & $<1000$ & $1 / 4096$ & AB3\#10 & $<63$ & 1/1024A \\
\hline $\mathrm{AB} 1 \# 10$ & $<1000$ & $1 / 1024$ & AB2\#13 & $<1000$ & $1 / 4096$ & AB3\#11 & $<63$ & $1 / 1024 \mathrm{~A}$ \\
\hline AB1\#11 & $<1000$ & $1 / 1024$ & AB3\#1 & $63-1000$ & $1 / 256 c$ & AB4\#1 & $63-1000$ & $1 / 256$ \\
\hline $\mathrm{AB} 1 \# 12$ & $<1000$ & $1 / 1024$ & AB3\#1 & $63-1000$ & $1 / 256 \mathrm{D}$ & $\mathrm{AB} 4 \# 2$ & $63-1000$ & $1 / 256$ \\
\hline AB1\#1 & $<1000$ & $1 / 4096$ & AB3\#2 & $63-1000$ & $1 / 256 c$ & $\mathrm{AB} 4 \# 3$ & $63-1000$ & $1 / 256$ \\
\hline $\mathrm{AB} 1 \# 2$ & $<1000$ & $1 / 4096$ & $\mathrm{AB} 3 \# 2$ & $63-1000$ & $1 / 256 \mathrm{D}$ & $\mathrm{AB} 4 \# 4$ & $63-1000$ & $1 / 256$ \\
\hline $\mathrm{AB} 1 \# 3$ & $<1000$ & $1 / 4096$ & AB3\#3 & $63-1000$ & $1 / 128$ & $\mathrm{AB} 4 \# 5$ & $63-1000$ & $1 / 256$ \\
\hline $\mathrm{AB} 1 \# 4$ & $<1000$ & $1 / 4096$ & AB3\#3 & $63-1000$ & $1 / 256 c$ & $\mathrm{AB} 4 \# 6$ & $63-1000$ & $1 / 256$ \\
\hline $\mathrm{AB} 1 \# 5$ & $<1000$ & $1 / 4096$ & AB3\#4 & $63-1000$ & $1 / 256 \mathrm{c}$ & $\mathrm{AB} 4 \# 7$ & $63-1000$ & $1 / 256$ \\
\hline $\mathrm{AB} 1 \# 6$ & $<1000$ & $1 / 4096$ & $\mathrm{AB} 3 \# 4$ & $63-1000$ & $1 / 1024 \mathrm{~A}$ & $\mathrm{AB} 4 \# 8$ & $63-1000$ & $1 / 256$ \\
\hline $\mathrm{AB} 1 \# 7$ & $<1000$ & $1 / 4096$ & $\mathrm{AB} 3 \# 5$ & $63-1000$ & $1 / 256 \mathrm{c}$ & AB4\#9 & $63-1000$ & $1 / 256$ \\
\hline $\mathrm{AB} 1 \# 8$ & $<1000$ & $1 / 4096$ & AB3\#6 & $63-1000$ & $1 / 256 \mathrm{c}$ & AB4\#10 & $63-1000$ & $1 / 256$ \\
\hline AB1\#9 & $<1000$ & $1 / 4096$ & AB3\#7 & $63-1000$ & $1 / 256 c$ & AB4\#11 & $63-1000$ & $1 / 256$ \\
\hline $\mathrm{AB} 1 \# 10$ & $<1000$ & $1 / 4096$ & $\mathrm{AB} 3 \# 8$ & $63-1000$ & $1 / 256 c$ & $\mathrm{AB} 4 \# 12$ & 63-1000 & $1 / 256$ \\
\hline AB1\#11 & $<1000$ & $1 / 4096$ & AB3\#9 & $63-1000$ & $1 / 256 \mathrm{c}$ & AB4\#1 & $<1000$ & $1 / 1024$ \\
\hline $\mathrm{AB} 1 \# 12$ & $<1000$ & $1 / 4096$ & AB3\#10 & $63-1000$ & $1 / 256 c$ & AB4\#2 & $<1000$ & $1 / 1024$ \\
\hline AB2\#1 & $63-1000$ & $1 / 256 c$ & AB3\#11 & $63-1000$ & $1 / 256 c$ & $\mathrm{AB} 4 \# 3$ & $<1000$ & $1 / 1024$ \\
\hline $\mathrm{AB} 2 \# 2$ & $63-1000$ & $1 / 256 c$ & AB3\#12 & $63-1000$ & $1 / 256 c$ & AB4\#4 & $<1000$ & $1 / 1024$ \\
\hline AB2\#3 & $63-1000$ & $1 / 256 \mathrm{c}$ & AB3\#13 & $63-1000$ & $1 / 256 \mathrm{c}$ & $\mathrm{AB} 4 \# 5$ & $<1000$ & $1 / 1024$ \\
\hline $\mathrm{AB} 2 \# 4$ & $63-1000$ & $1 / 256 c$ & AB3\#3 & $63-1000$ & $1 / 1024 a$ & $\mathrm{AB} 4 \# 6$ & $<1000$ & $1 / 1024$ \\
\hline $\mathrm{AB} 2 \# 5$ & $63-1000$ & $1 / 256 \mathrm{c}$ & AB3\#4 & $63-1000$ & $1 / 1024 d$ & $\mathrm{AB} 4 \# 7$ & $<1000$ & $1 / 1024$ \\
\hline $\mathrm{AB} 2 \# 6$ & $63-1000$ & $1 / 256 \mathrm{D}$ & $\mathrm{AB} 3 \# 8$ & $63-1000$ & $1 / 128$ & AB4\#8 & $<1000$ & $1 / 1024$ \\
\hline AB2\#7 & $63-1000$ & 1/256B & AB3\#8 & $63-1000$ & $1 / 1024 a$ & AB4\#9 & $<1000$ & $1 / 1024$ \\
\hline AB2\#7 & $63-1000$ & $1 / 256 \mathrm{C}$ & AB3\#9 & $63-1000$ & $1 / 128$ & AB4\#10 & $<1000$ & $1 / 1024$ \\
\hline $\mathrm{AB} 2 \# 8$ & $63-1000$ & $1 / 256 c$ & AB3\#9 & $63-1000$ & $1 / 1024 a$ & AB4\#11 & $<1000$ & $1 / 1024$ \\
\hline $\mathrm{AB} 2 \# 9$ & $63-1000$ & $2 / 1024$ & AB3\#10 & $63-1000$ & $1 / 1024 \mathrm{~A}$ & AB4\#1 & $<1000$ & $1 / 4096$ \\
\hline $\mathrm{AB} 2 \# 9$ & $63-1000$ & $1 / 1024$ & AB3\#10 & $63-1000$ & $1 / 1024 d$ & AB4\#2 & $<1000$ & $1 / 4096$ \\
\hline AB2\#9 & $63-1000$ & $1 / 1024 a$ & AB3\#11 & $63-1000$ & $1 / 1024 \mathrm{~A}$ & AB4\#3 & $<1000$ & $1 / 4096$ \\
\hline AB2\#10 & $63-1000$ & $1 / 256 \mathrm{D}$ & AB3\#7 & $<1000$ & $1 / 4096 \mathrm{D}$ & AB4\#4 & $<1000$ & $1 / 4096$ \\
\hline AB2\#11 & $63-1000$ & $1 / 256 \mathrm{D}$ & AB3\#1 & $<1000$ & $1 / 1024$ & $\mathrm{AB} 4 \# 5$ & $<1000$ & $1 / 4096$ \\
\hline AB2\#12 & $63-1000$ & $1 / 256 \mathrm{D}$ & AB3\#2 & $<1000$ & $1 / 1024$ & AB4\#6 & $<1000$ & $1 / 4096$ \\
\hline AB2\#13 & $63-1000$ & $1 / 256 b$ & AB3\#3 & $<1000$ & $1 / 1024$ & AB4\#7 & $<1000$ & $1 / 4096$ \\
\hline AB2\#13 & $63-1000$ & $1 / 256 \mathrm{c}$ & AB3\#4 & $<1000$ & $1 / 1024$ & $\mathrm{AB} 4 \# 8$ & $<1000$ & $1 / 4096$ \\
\hline AB2\#13 & $63-1000$ & $1 / 256 \mathrm{D}$ & $\mathrm{AB} 3 \# 5$ & $<1000$ & $1 / 1024$ & $\mathrm{AB} 4 \# 9$ & $<1000$ & $1 / 4096$ \\
\hline AB2\#1 & $<1000$ & $1 / 1024$ & $\mathrm{AB} 3 \# 6$ & $<1000$ & $1 / 1024$ & AB4\#10 & $<1000$ & $1 / 4096$ \\
\hline AB2\#2 & $<1000$ & $1 / 1024$ & AB3\#7 & $<1000$ & $1 / 1024$ & AB4\#11 & $<1000$ & $1 / 4096$ \\
\hline AB2\#3 & $<1000$ & $1 / 1024$ & AB3\#8 & $<1000$ & $1 / 1024$ & AB4\#1 & $<63$ & $2 / 1024$ \\
\hline AB2\#4 & $<1000$ & $1 / 1024$ & AB3\#9 & $<1000$ & $1 / 1024$ & AB4\#1 & $<63$ & $1 / 1024$ \\
\hline $\mathrm{AB} 2 \# 5$ & $<1000$ & $1 / 1024$ & AB3\#10 & $<1000$ & $1 / 1024$ & AB4\#1 & $<63$ & $1 / 1024$ \\
\hline AB2\#6 & $<1000$ & $1 / 1024$ & AB3\#11 & $<1000$ & $1 / 1024$ & AB4\#2 & $<63$ & $2 / 1024$ \\
\hline AB2\#7 & $<1000$ & $1 / 1024$ & AB3\#1 & $<1000$ & $1 / 4096$ & AB4\#2 & $<63$ & $1 / 1024$ \\
\hline AB2\#8 & $<1000$ & $1 / 1024$ & AB3\#2 & $<1000$ & $1 / 4096$ & AB4\#2 & $<63$ & $1 / 1024$ \\
\hline AB2\#9 & $<1000$ & $1 / 1024$ & AB3\#3 & $<1000$ & $1 / 4096$ & $\mathrm{AB} 4 \# 3$ & $<63$ & $2 / 1024$ \\
\hline AB2\#10 & $<1000$ & $1 / 1024$ & AB3\#4 & $<1000$ & $1 / 4096$ & $\mathrm{AB} 4 \# 3$ & $<63$ & $1 / 1024$ \\
\hline AB2\#11 & $<1000$ & $1 / 1024$ & $\mathrm{AB} 3 \# 5$ & $<1000$ & $1 / 4096$ & $\mathrm{AB} 4 \# 3$ & $<63$ & $1 / 1024$ \\
\hline AB2\#12 & $<1000$ & $1 / 1024$ & AB3\#6 & $<1000$ & $1 / 4096$ & AB4\#4 & $<63$ & $2 / 1024$ \\
\hline AB2\#13 & $<1000$ & $1 / 1024$ & AB3\#7 & $<1000$ & $1 / 4096$ & AB4\#4 & $<63$ & $1 / 1024$ \\
\hline AB2\#1 & $<1000$ & $1 / 4096$ & $\mathrm{AB} 3 \# 8$ & $<1000$ & $1 / 4096$ & AB4\#4 & $<63$ & $1 / 1024$ \\
\hline AB2\#2 & $<1000$ & $1 / 4096$ & AB3\#9 & $<1000$ & $1 / 4096$ & $\mathrm{AB} 4 \# 5$ & $<63$ & $2 / 1024$ \\
\hline AB2\#3 & $<1000$ & $1 / 4096$ & AB3\#10 & $<1000$ & $1 / 4096$ & AB4\#5 & $<63$ & $1 / 1024$ \\
\hline AB2\#4 & $<1000$ & $1 / 4096$ & AB3\#11 & $<1000$ & $1 / 4096$ & $\mathrm{AB} 4 \# 5$ & $<63$ & $1 / 1024$ \\
\hline
\end{tabular}


Kozo Takahashi, Hirofumi Asahi, Yusuke Okazaki, Jonaotaro Onodera, Hideto Tsutsui,

Takahito Ikenoue, Yoshiyuki Kanematsu, Seiji Tanaka and Shinya Iwasaki

Table 7 (Cont.)

\begin{tabular}{|c|c|c|c|c|c|c|c|c|}
\hline Sample ID & Size $(\mu \mathrm{m})$ & Aliquot size & Sample ID & Size $(\mu \mathrm{m})$ & Aliquot size & Sample ID & Size $(\mu \mathrm{m})$ & Aliquot size \\
\hline AB4\#6 & $<63$ & $2 / 1024$ & AB5\#12 & $<1000$ & $1 / 4096$ & AB6\#4 & $<1000$ & $1 / 4096$ \\
\hline AB4\#6 & $<63$ & $1 / 1024$ & AB5\#13 & $<1000$ & $1 / 4096$ & AB6\#5 & $<1000$ & $1 / 4096$ \\
\hline AB4\#6 & $<63$ & $1 / 1024$ & AB5\#1 & $<63$ & $1 / 1024$ & AB6\#6 & $<1000$ & $1 / 4096$ \\
\hline AB4\#7 & $<63$ & $2 / 1024$ & AB5\#2 & $<63$ & $1 / 1024$ & AB6\#7 & $<1000$ & $1 / 4096$ \\
\hline AB4\#7 & $<63$ & $1 / 1024$ & AB5\#3 & $<63$ & $1 / 1024$ & AB6\#8 & $<1000$ & $1 / 4096$ \\
\hline AB4\#7 & $<63$ & $1 / 1024$ & AB5\#4 & $<63$ & $1 / 1024$ & AB6\#9 & $<1000$ & $1 / 4096$ \\
\hline AB4\#8 & $<63$ & $2 / 1024$ & AB5\#5 & $<63$ & $1 / 1024$ & AB6\#10 & $<1000$ & $1 / 4096$ \\
\hline AB4\#8 & $<63$ & $1 / 1024$ & AB5\#6 & $<63$ & $1 / 1024$ & AB6\#11 & $<1000$ & $1 / 4096$ \\
\hline AB4\#8 & $<63$ & $1 / 1024$ & AB5\#7 & $<63$ & $1 / 1024$ & AB6\#12 & $<1000$ & $1 / 4096$ \\
\hline AB4\#9 & $<63$ & $2 / 1024$ & AB5\#8 & $<63$ & $1 / 256$ & AB6\#13 & $<1000$ & $1 / 4096$ \\
\hline AB4\#9 & $<63$ & $1 / 1024$ & AB5\#9 & $<63$ & $1 / 256$ & AB6\#1 & $<63$ & $1 / 256 \mathrm{~B}$ \\
\hline AB4\#9 & $<63$ & $1 / 1024$ & AB5\#10 & $<63$ & $1 / 256$ & AB6\#2 & $<63$ & $1 / 256 \mathrm{~B}$ \\
\hline AB4\#10 & $<63$ & $2 / 1024$ & AB5\#11 & $<63$ & $1 / 256$ & $\mathrm{AB} 6 \# 3$ & $<63$ & $1 / 256 \mathrm{~B}$ \\
\hline AB4\#10 & $<63$ & $1 / 1024$ & AB5\#12 & $<63$ & $1 / 1024$ & AB6\#4 & $<63$ & $1 / 256 \mathrm{~B}$ \\
\hline AB4\#10 & $<63$ & $1 / 1024$ & AB5\#13 & $<63$ & $1 / 1024$ & AB6\#5 & $<63$ & $1 / 256 \mathrm{~B}$ \\
\hline AB4\#11 & $<63$ & $2 / 1024$ & AB6\#1 & $63-1000$ & $1 / 256 \mathrm{~B}$ & AB6\#6 & $<63$ & $1 / 256 \mathrm{~B}$ \\
\hline AB4\#11 & $<63$ & $1 / 1024$ & AB6\#2 & $63-1000$ & $1 / 256 \mathrm{~B}$ & AB6\#7 & $<63$ & $1 / 256 \mathrm{~B}$ \\
\hline AB4\#11 & $<63$ & $1 / 1024$ & AB6\#3 & $63-1000$ & $1 / 256 \mathrm{~B}$ & AB6\#8 & $<63$ & $1 / 256 \mathrm{~B}$ \\
\hline AB4\#12 & $<63$ & $2 / 1024$ & AB6\#5 & $63-1000$ & $1 / 256 \mathrm{~B}$ & AB6\#9 & $<63$ & $1 / 256 \mathrm{~B}$ \\
\hline AB4\#12 & $<63$ & $1 / 1024$ & AB6\#6 & $63-1000$ & $1 / 256 \mathrm{~B}$ & AB6\#10 & $<63$ & $1 / 256 \mathrm{~B}$ \\
\hline AB4\#12 & $<63$ & $1 / 1024$ & AB6\#7 & $63-1000$ & $1 / 256 \mathrm{~B}$ & AB6\#11 & $<63$ & $1 / 256 \mathrm{~B}$ \\
\hline AB5\#1 & $63-1000$ & $1 / 256$ & AB6\#8 & $63-1000$ & $1 / 256 \mathrm{~B}$ & AB6\#12 & $<63$ & $1 / 256 \mathrm{~B}$ \\
\hline AB5\#2 & $63-1000$ & $1 / 256$ & AB6\#9 & $63-1000$ & $1 / 256 \mathrm{~B}$ & AB6\#13 & $<63$ & $1 / 256 \mathrm{~B}$ \\
\hline AB5\#3 & $63-1000$ & $1 / 256$ & AB6\#10 & $63-1000$ & $1 / 256 \mathrm{~B}$ & AB6\#1 & $<63$ & 1/1024A \\
\hline AB5\#4 & $63-1000$ & $1 / 256$ & AB6\#11 & $63-1000$ & $1 / 256 \mathrm{~B}$ & AB6\#2 & $<63$ & 1/1024B \\
\hline $\mathrm{AB} 5 \# 5$ & $63-1000$ & $1 / 256$ & AB6\#12 & $63-1000$ & $1 / 256 \mathrm{~B}$ & $\mathrm{AB} 6 \# 3$ & $<63$ & 1/1024B \\
\hline AB5\#6 & $63-1000$ & $1 / 256$ & AB6\#13 & $63-1000$ & $1 / 256 \mathrm{~B}$ & AB6\#4 & $<63$ & 1/1024B \\
\hline AB5\#7 & $63-1000$ & $1 / 256$ & AB6\#1 & $63-1000$ & $1 / 1024 \mathrm{~A}$ & AB6\#5 & $<63$ & 1/1024B \\
\hline AB5\#8 & $63-1000$ & $1 / 256$ & AB6\#2 & $63-1000$ & 1/1024B & AB6\#6 & $<63$ & 1/1024B \\
\hline AB5\#9 & $63-1000$ & $1 / 256$ & AB6\#3 & $63-1000$ & 1/1024B & AB6\#7 & $<63$ & $1 / 1024 \mathrm{~A}$ \\
\hline AB5\#10 & $63-1000$ & $1 / 256$ & AB6\#4 & $63-1000$ & 1/1024B & AB6\#8 & $<63$ & 1/1024B \\
\hline AB5\#11 & $63-1000$ & $1 / 256$ & AB6\#5 & $63-1000$ & 1/1024B & AB6\#9 & $<63$ & 1/1024B \\
\hline AB5\#12 & $63-1000$ & $1 / 256$ & AB6\#6 & $63-1000$ & 1/1024B & AB6\#10 & $<63$ & 1/1024B \\
\hline AB5\#13 & $63-1000$ & $1 / 1024$ & AB6\#7 & $63-1000$ & 1/1024B & AB6\#11 & $<63$ & 1/1024B \\
\hline AB5\#1 & $<1000$ & $1 / 1024$ & AB6\#8 & $63-1000$ & 1/1024B & AB6\#12 & $<63$ & 1/1024B \\
\hline AB5\#2 & $<1000$ & $1 / 1024$ & AB6\#9 & $63-1000$ & 1/1024B & AB6\#13 & $<63$ & 1/1024B \\
\hline AB5\#3 & $<1000$ & $1 / 1024$ & AB6\#10 & $63-1000$ & 1/1024B & AB6\#9 & $<63$ & 1/4096B \\
\hline AB5\#4 & $<1000$ & $1 / 1024$ & AB6\#11 & $63-1000$ & 1/1024B & AB6\#10 & $<63$ & 1/4096B \\
\hline AB5\#5 & $<1000$ & $1 / 1024$ & AB6\#12 & $63-1000$ & 1/1024B & AB7\#1 & $63-1000$ & $1 / 256 \mathrm{~B}$ \\
\hline AB5\#6 & $<1000$ & $1 / 1024$ & AB6\#13 & $63-1000$ & 1/1024B & $\mathrm{AB} 7 \# 2$ & $63-1000$ & $1 / 256 \mathrm{~B}$ \\
\hline AB5\#7 & $<1000$ & $1 / 1024$ & AB6\#12 & $<1000$ & $1 / 16384 \mathrm{~A}$ & $\mathrm{AB} 7 \# 3$ & $63-1000$ & $1 / 256 \mathrm{~B}$ \\
\hline AB5\#8 & $<1000$ & $1 / 1024$ & AB6\#13 & $<1000$ & $1 / 16384 \mathrm{~A}$ & $\mathrm{AB} 7 \# 4$ & $63-1000$ & $1 / 256 \mathrm{~B}$ \\
\hline AB5\#9 & $<1000$ & $1 / 1024$ & AB6\#1 & $<1000$ & $1 / 1024$ & $\mathrm{AB} 7 \# 5$ & $63-1000$ & $1 / 256 \mathrm{~B}$ \\
\hline AB5\#10 & $<1000$ & $1 / 1024$ & AB6\#2 & $<1000$ & $1 / 1024$ & $\mathrm{AB} 7 \# 6$ & $63-1000$ & $1 / 256 \mathrm{~B}$ \\
\hline AB5\#11 & $<1000$ & $1 / 1024$ & AB6\#3 & $<1000$ & $1 / 1024$ & $\mathrm{AB} 7 \# 7$ & $63-1000$ & $1 / 256 \mathrm{~B}$ \\
\hline AB5\#12 & $<1000$ & $1 / 1024$ & AB6\#4 & $<1000$ & $1 / 1024$ & $\mathrm{AB} 7 \# 8$ & $63-1000$ & $1 / 256 \mathrm{~B}$ \\
\hline AB5\#13 & $<1000$ & $1 / 1024$ & AB6\#5 & $<1000$ & $1 / 1024$ & $\mathrm{AB} 7 \# 9$ & $63-1000$ & $1 / 256 \mathrm{~B}$ \\
\hline AB5\#1 & $<1000$ & $1 / 4096$ & AB6\#6 & $<1000$ & $1 / 1024$ & AB7\#10 & $63-1000$ & $1 / 256 \mathrm{~B}$ \\
\hline AB5\#2 & $<1000$ & $1 / 4096$ & AB6\#7 & $<1000$ & $1 / 1024$ & AB7\#11 & $63-1000$ & $1 / 256 \mathrm{~B}$ \\
\hline AB5\#3 & $<1000$ & $1 / 4096$ & AB6\#8 & $<1000$ & $1 / 1024$ & $\mathrm{AB} 7 \# 12$ & $63-1000$ & $1 / 256 \mathrm{~B}$ \\
\hline AB5\#4 & $<1000$ & $1 / 4096$ & AB6\#9 & $<1000$ & $1 / 1024$ & AB7\#13 & $63-1000$ & $1 / 256 \mathrm{~B}$ \\
\hline AB5\#5 & $<1000$ & $1 / 4096$ & AB6\#10 & $<1000$ & $1 / 1024$ & AB7\#1 & $<1000$ & $1 / 1024$ \\
\hline AB5\#6 & $<1000$ & $1 / 4096$ & AB6\#11 & $<1000$ & $1 / 1024$ & $\mathrm{AB} 7 \# 2$ & $<1000$ & $1 / 1024$ \\
\hline AB5\#7 & $<1000$ & $1 / 4096$ & AB6\#12 & $<1000$ & $1 / 1024$ & $\mathrm{AB} 7 \# 3$ & $<1000$ & $1 / 1024$ \\
\hline AB5\#8 & $<1000$ & $1 / 4096$ & AB6\#13 & $<1000$ & $1 / 1024$ & $\mathrm{AB} 7 \# 4$ & $<1000$ & $1 / 1024$ \\
\hline AB5\#9 & $<1000$ & $1 / 4096$ & AB6\#1 & $<1000$ & $1 / 4096$ & $\mathrm{AB} 7 \# 5$ & $<1000$ & $1 / 1024$ \\
\hline AB5\#10 & $<1000$ & $1 / 4096$ & AB6\#2 & $<1000$ & $1 / 4096$ & $\mathrm{AB} 7 \# 6$ & $<1000$ & $1 / 1024$ \\
\hline AB5\#11 & $<1000$ & $1 / 4096$ & AB6\#3 & $<1000$ & $1 / 4096$ & $\mathrm{AB} 7 \# 7$ & $<1000$ & $1 / 1024$ \\
\hline
\end{tabular}


Table 7 (Cont.)

\begin{tabular}{|c|c|c|c|c|c|c|c|c|}
\hline Sample ID & Size $(\mu \mathrm{m})$ & Aliquot size & Sample ID & Size $(\mu \mathrm{m})$ & Aliquot size & Sample ID & Size $(\mu \mathrm{m})$ & Aliquot size \\
\hline $\mathrm{AB} 7 \# 8$ & $<1000$ & $1 / 1024$ & AB8\#4 & $<1000$ & $1 / 4096$ & AB9\#10 & $<63$ & $1 / 1024$ \\
\hline AB7\#9 & $<1000$ & $1 / 1024$ & AB8\#5 & $<1000$ & $1 / 4096$ & AB9\#11 & $<63$ & $1 / 1024$ \\
\hline $\mathrm{AB} 7 \# 10$ & $<1000$ & $1 / 1024$ & AB8\#6 & $<1000$ & $1 / 4096$ & AB9\#12 & $<63$ & $1 / 1024$ \\
\hline AB7\#11 & $<1000$ & $1 / 1024$ & AB8\#7 & $<1000$ & $1 / 4096$ & AB9\#13 & $<63$ & $1 / 1024$ \\
\hline $\mathrm{AB} 7 \# 12$ & $<1000$ & $1 / 1024$ & AB8\#8 & $<1000$ & $1 / 4096$ & AB9\#1 & $<63$ & $1 / 4096$ \\
\hline AB7\#13 & $<1000$ & $1 / 1024$ & AB8\#9 & $<1000$ & $1 / 4096$ & AB9\#2 & $<63$ & $1 / 4096$ \\
\hline AB7\#1 & $<1000$ & $1 / 4096$ & AB8\#10 & $<1000$ & $1 / 4096$ & AB9\#3 & $<63$ & $1 / 4096$ \\
\hline $\mathrm{AB} 7 \# 2$ & $<1000$ & $1 / 4096$ & AB8\#11 & $<1000$ & $1 / 4096$ & AB9\#4 & $<63$ & $1 / 4096$ \\
\hline $\mathrm{AB} 7 \# 3$ & $<1000$ & $1 / 4096$ & AB8\#12 & $<1000$ & $1 / 4096$ & AB9\#5 & $<63$ & $1 / 4096$ \\
\hline $\mathrm{AB} 7 \# 4$ & $<1000$ & $1 / 4096$ & AB8\#13 & $<1000$ & $1 / 4096$ & AB9\#6 & $<63$ & $1 / 4096$ \\
\hline $\mathrm{AB} 7 \# 5$ & $<1000$ & $1 / 4096$ & AB8\#1 & $<63$ & $1 / 4096$ & AB9\#7 & $<63$ & $1 / 4096$ \\
\hline $\mathrm{AB} 7 \# 6$ & $<1000$ & $1 / 4096$ & AB8\#2 & $<63$ & $1 / 4096$ & AB9\#8 & $<63$ & $1 / 4096$ \\
\hline $\mathrm{AB} 7 \# 7$ & $<1000$ & $1 / 4096$ & AB8\#3 & $<63$ & $1 / 4096$ & AB9\#9 & $<63$ & $1 / 4096$ \\
\hline $\mathrm{AB} 7 \# 8$ & $<1000$ & $1 / 4096$ & AB8\#4 & $<63$ & $1 / 4096$ & AB9\#10 & $<63$ & $1 / 4096$ \\
\hline AB7\#9 & $<1000$ & $1 / 4096$ & AB8\#5 & $<63$ & $1 / 4096$ & AB9\#11 & $<63$ & $1 / 4096$ \\
\hline AB7\#10 & $<1000$ & $1 / 4096$ & AB8\#6 & $<63$ & $1 / 4096$ & AB9\#12 & $<63$ & $1 / 4096$ \\
\hline AB7\#11 & $<1000$ & $1 / 4096$ & AB8\#7 & $<63$ & $1 / 4096$ & AB9\#13 & $<63$ & $1 / 4096$ \\
\hline $\mathrm{AB} 7 \# 12$ & $<1000$ & $1 / 4096$ & AB8\#8 & $<63$ & $1 / 4096$ & AB14\#1 & $63-1000$ & $1 / 64$ \\
\hline AB7\#13 & $<1000$ & $1 / 4096$ & AB8\#9 & $<63$ & $1 / 4096$ & AB14\#2 & $63-1000$ & $1 / 64$ \\
\hline AB7\#1 & $<63$ & $1 / 1024$ & AB8\#10 & $<63$ & $1 / 4096$ & AB14\#3 & $63-1000$ & $1 / 256$ \\
\hline $\mathrm{AB} 7 \# 5$ & $<63$ & $1 / 1024$ & AB8\#11 & $<63$ & $1 / 4096$ & $\mathrm{AB} 14 \# 4$ & $63-1000$ & $1 / 256$ \\
\hline $\mathrm{AB} 7 \# 6$ & $<63$ & $1 / 1024$ & AB8\#12 & $<63$ & $1 / 4096$ & $\mathrm{AB} 14 \# 5$ & $63-1000$ & $1 / 256$ \\
\hline $\mathrm{AB} 7 \# 7$ & $<63$ & $1 / 1024$ & AB8\#13 & $<63$ & $1 / 4096$ & AB14\#6 & $63-1000$ & $1 / 256$ \\
\hline $\mathrm{AB} 7 \# 8$ & $<63$ & $1 / 1024$ & AB9\#1 & $63-1000$ & $1 / 256 \mathrm{C}$ & AB14\#7 & $63-1000$ & $1 / 256$ \\
\hline AB7\#9 & $<63$ & $1 / 1024$ & AB9\#2 & $63-1000$ & $1 / 256 \mathrm{C}$ & $\mathrm{AB} 14 \# 8$ & $63-1000$ & $1 / 256$ \\
\hline AB7\#10 & $<63$ & $1 / 1024$ & AB9\#3 & $63-1000$ & $1 / 256 \mathrm{C}$ & AB14\#9 & $63-1000$ & $1 / 256$ \\
\hline AB7\#11 & $<63$ & $1 / 1024$ & AB9\#4 & $63-1000$ & $1 / 256 \mathrm{C}$ & $\mathrm{AB} 14 \# 10$ & $63-1000$ & $1 / 256$ \\
\hline $\mathrm{AB} 7 \# 12$ & $<63$ & $1 / 1024$ & AB9\#5 & $63-1000$ & $1 / 256 \mathrm{C}$ & AB14\#11 & $63-1000$ & $1 / 64$ \\
\hline $\mathrm{AB} 7 \# 13$ & $<63$ & $1 / 1024$ & AB9\#6 & $63-1000$ & $1 / 256 \mathrm{C}$ & AB14\#12 & $63-1000$ & $1 / 64$ \\
\hline AB8\#1 & $63-1000$ & $1 / 256 \mathrm{~B}$ & AB9\#7 & $63-1000$ & $1 / 256 \mathrm{C}$ & $\mathrm{AB} 14 \# 13$ & $63-1000$ & $1 / 64$ \\
\hline AB8\#2 & $63-1000$ & $1 / 256 \mathrm{~B}$ & AB9\#8 & $63-1000$ & $1 / 256 \mathrm{C}$ & $\mathrm{AB} 14 \# 14$ & $63-1000$ & $1 / 64$ \\
\hline AB8\#3 & $63-1000$ & $1 / 256 \mathrm{~B}$ & AB9\#9 & $63-1000$ & $1 / 256 \mathrm{C}$ & AB14\#15 & $63-1000$ & $1 / 256$ \\
\hline $\mathrm{AB} 8 \# 4$ & $63-1000$ & $1 / 256 \mathrm{~B}$ & AB9\#10 & $63-1000$ & $1 / 256 \mathrm{C}$ & AB14\#16 & $63-1000$ & $1 / 256$ \\
\hline AB8\#5 & $63-1000$ & $1 / 256 \mathrm{~B}$ & AB9\#11 & $63-1000$ & $1 / 256 \mathrm{C}$ & $\mathrm{AB} 14 \# 17$ & $63-1000$ & $1 / 256$ \\
\hline AB8\#6 & $63-1000$ & $1 / 256 \mathrm{~B}$ & AB9\#12 & $63-1000$ & $1 / 256 \mathrm{C}$ & AB14\#18 & $63-1000$ & $1 / 256$ \\
\hline AB8\#7 & $63-1000$ & $1 / 256 \mathrm{~B}$ & AB9\#13 & $63-1000$ & $1 / 256 \mathrm{C}$ & AB14\#19 & $63-1000$ & $1 / 256$ \\
\hline AB8\#8 & $63-1000$ & $1 / 256 \mathrm{~B}$ & AB9\#1 & $<1000$ & $1 / 4096$ & $\mathrm{AB} 14 \# 20$ & $63-1000$ & $1 / 16$ \\
\hline AB8\#9 & $63-1000$ & $1 / 256 \mathrm{~B}$ & AB9\#2 & $<1000$ & $1 / 4096$ & AB14\#21 & $63-1000$ & $1 / 16$ \\
\hline AB8\#10 & $63-1000$ & $1 / 256 \mathrm{~B}$ & AB9\#3 & $<1000$ & $1 / 4096$ & AB14\#1 & $<1000$ & $1 / 4096$ \\
\hline AB8\#11 & $63-1000$ & $1 / 256 \mathrm{~B}$ & AB9\#4 & $<1000$ & $1 / 4096$ & AB14\#2 & $<1000$ & $1 / 4096$ \\
\hline AB8\#12 & $63-1000$ & $1 / 256 \mathrm{~B}$ & AB9\#5 & $<1000$ & $1 / 4096$ & $\mathrm{AB} 14 \# 3$ & $<1000$ & $1 / 4096$ \\
\hline AB8\#13 & $63-1000$ & $1 / 256 \mathrm{~B}$ & AB9\#6 & $<1000$ & $1 / 4096$ & AB14\#4 & $<1000$ & $1 / 4096$ \\
\hline AB8\#1 & $<1000$ & $1 / 1024$ & AB9\#7 & $<1000$ & $1 / 4096$ & $\mathrm{AB} 14 \# 5$ & $<1000$ & $1 / 4096$ \\
\hline AB8\#2 & $<1000$ & $1 / 1024$ & AB9\#8 & $<1000$ & $1 / 4096$ & AB14\#6 & $<1000$ & $1 / 4096$ \\
\hline AB8\#3 & $<1000$ & $1 / 1024$ & AB9\#9 & $<1000$ & $1 / 4096$ & AB14\#7 & $<1000$ & $1 / 4096$ \\
\hline AB8\#4 & $<1000$ & $1 / 1024$ & AB9\#10 & $<1000$ & $1 / 4096$ & AB14\#8 & $<1000$ & $1 / 4096$ \\
\hline AB8\#5 & $<1000$ & $1 / 1024$ & AB9\#11 & $<1000$ & $1 / 4096$ & $\mathrm{AB} 14 \# 9$ & $<1000$ & $1 / 4096$ \\
\hline AB8\#6 & $<1000$ & $1 / 1024$ & AB9\#12 & $<1000$ & $1 / 4096$ & AB14\#10 & $<1000$ & $1 / 4096$ \\
\hline AB8\#7 & $<1000$ & $1 / 1024$ & AB9\#13 & $<1000$ & $1 / 4096$ & AB14\#11 & $<1000$ & $1 / 4096$ \\
\hline AB8\#8 & $<1000$ & $1 / 1024$ & AB9\#1 & $<63$ & $1 / 1024$ & $\mathrm{AB} 14 \# 12$ & $<1000$ & $1 / 4096$ \\
\hline AB8\#9 & $<1000$ & $1 / 1024$ & AB9\#2 & $<63$ & $1 / 1024$ & $\mathrm{AB} 14 \# 13$ & $<1000$ & $1 / 4096$ \\
\hline AB8\#10 & $<1000$ & $1 / 1024$ & AB9\#3 & $<63$ & $1 / 1024$ & AB14\#14 & $<1000$ & $1 / 4096$ \\
\hline AB8\#11 & $<1000$ & $1 / 1024$ & AB9\#4 & $<63$ & $1 / 1024$ & AB14\#15 & $<1000$ & $1 / 4096$ \\
\hline AB8\#12 & $<1000$ & $1 / 1024$ & AB9\#5 & $<63$ & $1 / 1024$ & $\mathrm{AB} 14 \# 16$ & $<1000$ & $1 / 4096$ \\
\hline AB8\#13 & $<1000$ & $1 / 1024$ & AB9\#6 & $<63$ & $1 / 1024$ & AB14\#17 & $<1000$ & $1 / 4096$ \\
\hline AB8\#1 & $<1000$ & $1 / 4096$ & AB9\#7 & $<63$ & $1 / 1024$ & AB14\#18 & $<1000$ & $1 / 4096$ \\
\hline AB8\#2 & $<1000$ & $1 / 4096$ & AB9\#8 & $<63$ & $1 / 1024$ & AB14\#19 & $<1000$ & $1 / 4096$ \\
\hline AB8\#3 & $<1000$ & $1 / 4096$ & AB9\#9 & $<63$ & $1 / 1024$ & AB14\#1 & $<63$ & $1 / 320$ \\
\hline
\end{tabular}


Kozo Takahashi, Hirofumi Asahi, Yusuke Okazaki, Jonaotaro Onodera, Hideto Tsutsui,

Takahito Ikenoue, Yoshiyuki Kanematsu, Seiji Tanaka and Shinya Iwasaki

Table 7 (Cont.)

\begin{tabular}{|c|c|c|c|c|c|c|c|c|}
\hline Sample ID & Size $(\mu \mathrm{m})$ & Aliquot size & $\begin{array}{c}\text { Sample ID } \\
\end{array}$ & Size $(\mu \mathrm{m})$ & Aliquot size & $\begin{array}{c}\text { Sample ID } \\
\end{array}$ & Size $(\mu \mathrm{m})$ & Aliquot size \\
\hline $\mathrm{AB} 14 \# 2$ & $<63$ & $1 / 320$ & AB16\#9 & $63-1000$ & $1 / 256$ & $\mathrm{AB} 18 \# 4$ & $<1000$ & $1 / 4096$ \\
\hline AB14\#3 & $<63$ & $1 / 1280$ & AB16\#10 & $63-1000$ & $1 / 256$ & AB18\#5 & $<1000$ & $1 / 4096$ \\
\hline AB14\#4 & $<63$ & $1 / 1280$ & AB16\#11 & $63-1000$ & $1 / 64$ & AB18\#6 & $<1000$ & $1 / 4096$ \\
\hline $\mathrm{AB} 14 \# 5$ & $<63$ & $1 / 1280$ & AB16\#12 & $63-1000$ & $1 / 64$ & AB18\#7 & $<1000$ & $1 / 4096$ \\
\hline AB14\#6 & $<63$ & $1 / 1280$ & AB16\#13 & $63-1000$ & $1 / 64$ & AB18\#8 & $<1000$ & $1 / 4096$ \\
\hline AB14\#7 & $<63$ & $1 / 1280$ & AB16\#14 & $63-1000$ & $1 / 64$ & AB18\#9 & $<1000$ & $1 / 4096$ \\
\hline $\mathrm{AB} 14 \# 8$ & $<63$ & $1 / 1280$ & AB16\#15 & $63-1000$ & $1 / 64$ & AB18\#10 & $<1000$ & $1 / 4096$ \\
\hline AB14\#9 & $<63$ & $1 / 1280$ & AB16\#16 & $63-1000$ & $1 / 64$ & AB18\#11 & $<1000$ & $1 / 4096$ \\
\hline $\mathrm{AB} 14 \# 10$ & $<63$ & $1 / 1280$ & AB16\#17 & $63-1000$ & $1 / 64$ & AB18\#12 & $<1000$ & $1 / 4096$ \\
\hline AB14\#11 & $<63$ & $1 / 320$ & AB16\#18 & $63-1000$ & $1 / 64$ & AB18\#13 & $<1000$ & $1 / 4096$ \\
\hline AB14\#12 & $<63$ & $1 / 320$ & AB16\#19 & $63-1000$ & $1 / 256$ & AB18\#14 & $<1000$ & $1 / 4096$ \\
\hline AB14\#13 & $<63$ & $1 / 320$ & $\mathrm{AB} 16 \# 20$ & $63-1000$ & $1 / 64$ & AB18\#15 & $<1000$ & $1 / 4096$ \\
\hline AB14\#14 & $<63$ & $1 / 320$ & AB16\#21 & $63-1000$ & $1 / 64$ & AB19\#1 & $<1000$ & $1 / 4096$ \\
\hline AB14\#15 & $<63$ & $1 / 1280$ & AB16\#1 & $<1000$ & $1 / 4096$ & AB19\#2 & $<1000$ & $1 / 4096$ \\
\hline AB14\#16 & $<63$ & $1 / 1280$ & AB16\#2 & $<1000$ & $1 / 4096$ & AB19\#3 & $<1000$ & $1 / 4096$ \\
\hline AB14\#17 & $<63$ & $1 / 1280$ & AB16\#3 & $<1000$ & $1 / 4096$ & $\mathrm{AB} 1 \# 2$ & $63-125$ & $1 / 64$ \\
\hline AB14\#18 & $<63$ & $1 / 1280$ & AB16\#4 & $<1000$ & $1 / 4096$ & $\mathrm{AB} 1 \# 3$ & $63-125$ & $1 / 64$ \\
\hline AB14\#19 & $<63$ & $1 / 1280$ & AB16\#5 & $<1000$ & $1 / 4096$ & $\mathrm{AB} 1 \# 4$ & $63-125$ & $1 / 64$ \\
\hline AB14\#20 & $<63$ & $1 / 16$ & AB16\#6 & $<1000$ & $1 / 4096$ & $\mathrm{AB} 1 \# 6$ & $63-125$ & $1 / 64$ \\
\hline AB14\#21 & $<63$ & $1 / 16$ & AB16\#7 & $<1000$ & $1 / 4096$ & $\mathrm{AB} 1 \# 7$ & $63-125$ & $1 / 64$ \\
\hline AB15\#1 & $63-1000$ & $1 / 256$ & AB16\#8 & $<1000$ & $1 / 4096$ & $\mathrm{AB} 1 \# 8$ & $63-125$ & $1 / 64$ \\
\hline AB15\#2 & $63-1000$ & $1 / 256$ & AB16\#9 & $<1000$ & $1 / 4096$ & $\mathrm{AB} 1 \# 9$ & $63-125$ & $1 / 64$ \\
\hline AB15\#3 & $63-1000$ & $1 / 256$ & AB16\#10 & $<1000$ & $1 / 4096$ & $\mathrm{AB} 1 \# 10$ & $63-125$ & $1 / 64$ \\
\hline AB15\#4 & $63-1000$ & $1 / 64$ & AB16\#11 & $<1000$ & $1 / 4096$ & $\mathrm{AB} 1 \# 11$ & $63-125$ & $1 / 64$ \\
\hline AB15\#5 & $63-1000$ & $1 / 64$ & $\mathrm{AB} 16 \# 12$ & $<1000$ & $1 / 4096$ & $\mathrm{AB} 1 \# 12$ & $63-125$ & $1 / 64$ \\
\hline AB15\#6 & $63-1000$ & $1 / 64$ & AB16\#13 & $<1000$ & $1 / 4096$ & AB2\#1 & $63-125$ & $1 / 64$ \\
\hline AB15\#7 & $63-1000$ & $1 / 64$ & AB16\#14 & $<1000$ & $1 / 4096$ & AB2\#2 & $63-125$ & $1 / 64$ \\
\hline AB15\#8 & $63-1000$ & $1 / 64$ & AB16\#15 & $<1000$ & $1 / 4096$ & $\mathrm{AB} 2 \# 3$ & $63-125$ & $1 / 64$ \\
\hline AB15\#9 & $63-1000$ & $1 / 256$ & AB16\#16 & $<1000$ & $1 / 4096$ & $\mathrm{AB} 2 \# 4$ & $63-125$ & $1 / 64$ \\
\hline AB15\#10 & $63-1000$ & $1 / 64$ & AB16\#17 & $<1000$ & $1 / 4096$ & $\mathrm{AB} 2 \# 5$ & $63-125$ & $1 / 64$ \\
\hline AB15\#1 & $<1000$ & $1 / 4096$ & AB16\#18 & $<1000$ & $1 / 4096$ & $\mathrm{AB} 2 \# 6$ & $63-125$ & $1 / 64$ \\
\hline AB15\#2 & $<1000$ & $1 / 4096$ & AB16\#19 & $<1000$ & $1 / 4096$ & $\mathrm{AB} 2 \# 7$ & $63-125$ & $1 / 64$ \\
\hline AB15\#3 & $<1000$ & $1 / 4096$ & $\mathrm{AB} 16 \# 20$ & $<1000$ & $1 / 4096$ & $\mathrm{AB} 2 \# 8$ & $63-125$ & $1 / 64$ \\
\hline AB15\#4 & $<1000$ & $1 / 4096$ & $\mathrm{AB} 16 \# 21$ & $<1000$ & $1 / 4096$ & AB2\#9 & $63-125$ & $1 / 64$ \\
\hline AB15\#5 & $<1000$ & $1 / 4096$ & AB16\#1 & $<63$ & $1 / 1280$ & $\mathrm{AB} 2 \# 12$ & $63-125$ & $1 / 64$ \\
\hline AB15\#6 & $<1000$ & $1 / 4096$ & AB16\#2 & $<63$ & $1 / 1280$ & $\mathrm{AB} 2 \# 13$ & $63-125$ & $1 / 64$ \\
\hline AB15\#7 & $<1000$ & $1 / 4096$ & AB16\#3 & $<63$ & $1 / 1280$ & AB3\#1 & $63-125$ & $1 / 64$ \\
\hline AB15\#8 & $<1000$ & $1 / 4096$ & AB16\#4 & $<63$ & $1 / 1280$ & AB3\#2 & $63-125$ & $1 / 64$ \\
\hline AB15\#9 & $<1000$ & $1 / 4096$ & AB16\#5 & $<63$ & $1 / 1280$ & $\mathrm{AB} 3 \# 3$ & $63-125$ & $1 / 64$ \\
\hline AB15\#10 & $<1000$ & $1 / 4096$ & AB16\#6 & $<63$ & $1 / 1280$ & AB3\#4 & $63-125$ & $1 / 64$ \\
\hline AB15\#1 & $<63$ & $1 / 1280$ & $\mathrm{AB} 16 \# 7$ & $<63$ & $1 / 1280$ & $\mathrm{AB} 3 \# 5$ & $63-125$ & $1 / 64$ \\
\hline AB15\#2 & $<63$ & $1 / 1280$ & AB16\#8 & $<63$ & $1 / 1280$ & $\mathrm{AB} 3 \# 6$ & $63-125$ & $1 / 64$ \\
\hline AB15\#3 & $<63$ & $1 / 1280$ & AB16\#9 & $<63$ & $1 / 1280$ & $\mathrm{AB} 3 \# 7$ & $63-125$ & $1 / 64$ \\
\hline AB15\#4 & $<63$ & $1 / 320$ & AB16\#10 & $<63$ & $1 / 1280$ & AB3\#8 & $63-125$ & $1 / 64$ \\
\hline AB15\#5 & $<63$ & $1 / 320$ & AB16\#11 & $<63$ & $1 / 320$ & AB3\#9 & $63-125$ & $1 / 64$ \\
\hline AB15\#6 & $<63$ & $1 / 320$ & AB16\#12 & $<63$ & $1 / 320$ & AB3\#10 & $63-125$ & $1 / 64$ \\
\hline AB15\#7 & $<63$ & $1 / 320$ & $\mathrm{AB} 16 \# 13$ & $<63$ & $1 / 320$ & AB3\#11 & $63-125$ & $1 / 64$ \\
\hline AB15\#8 & $<63$ & $1 / 320$ & AB16\#14 & $<63$ & $1 / 320$ & $\overline{\mathrm{AB}} 4 \# 1$ & $63-125$ & $1 / 64$ \\
\hline AB15\#9 & $<63$ & $1 / 1280$ & AB16\#15 & $<63$ & $1 / 320$ & $\mathrm{AB} 4 \# 2$ & $63-125$ & $1 / 64$ \\
\hline AB15\#10 & $<63$ & $1 / 320$ & AB16\#16 & $<63$ & $1 / 320$ & $\mathrm{AB} 4 \# 4$ & $63-125$ & $1 / 64$ \\
\hline AB16\#1 & $63-1000$ & $1 / 256$ & AB16\#17 & $<63$ & $1 / 320$ & $\mathrm{AB} 4 \# 5$ & $63-125$ & $1 / 64$ \\
\hline AB16\#2 & $63-1000$ & $1 / 256$ & AB16\#18 & $<63$ & $1 / 320$ & $\mathrm{AB} 4 \# 6$ & $63-125$ & $1 / 64$ \\
\hline AB16\#3 & $63-1000$ & $1 / 256$ & AB16\#19 & $<63$ & $1 / 1280$ & $\mathrm{AB} 4 \# 7$ & $63-125$ & $1 / 64$ \\
\hline AB16\#4 & $63-1000$ & $1 / 256$ & $\mathrm{AB} 16 \# 20$ & $<63$ & $1 / 320$ & $\mathrm{AB} 4 \# 8$ & $63-125$ & $1 / 64$ \\
\hline AB16\#5 & $63-1000$ & $1 / 256$ & $\mathrm{AB} 16 \# 21$ & $<63$ & $1 / 320$ & AB4\#9 & $63-125$ & $1 / 64$ \\
\hline AB16\#6 & $63-1000$ & $1 / 256$ & $\overline{\mathrm{AB} 18 \# 1}$ & $<1000$ & $1 / 4096$ & $\mathrm{AB} 4 \# 10$ & $63-125$ & $1 / 64$ \\
\hline AB16\#7 & $63-1000$ & $1 / 256$ & AB18\#2 & $<1000$ & $1 / 4096$ & AB4\#11 & $63-125$ & $1 / 64$ \\
\hline AB16\#8 & $63-1000$ & $1 / 256$ & AB18\#3 & $<1000$ & $1 / 4096$ & & & \\
\hline
\end{tabular}

